\title{
On the [2,3] Sigmatropic Rearrangement of Allylic Nitro Compounds
}

\author{
Celia Alameda-Angulo, Béatrice Quiclet-Sire, Elmar Schmidt and Samir Z. Zard* \\ Laboratoire de Synthèse Organique associé au CNRS \\ Ecole Polytechnique, 91128 Palaiseau Cedex (France)
}

\section{Supporting Information}

General Methods: Reactions were carried out under an inert gas, with magnetic stirring and degassed solvents when necessary. Tetrahydrofuran (THF) and diethyl ether $\left(\mathrm{Et}_{2} \mathrm{O}\right)$ were distilled from sodium/benzophenone under nitrogen and dichloromethane $\left(\mathrm{CH}_{2} \mathrm{Cl}_{2}\right)$ was distilled from $\mathrm{CaH}_{2}$ under nitrogen. Merck Geduran SI $60 \AA$ silica gel $(35-70 \mu \mathrm{m})$ was used for column chromatography. PE, EE, and EA are petroleum ether, diethyl ether and ethyl acetate respectively. IR spectra were recorded with a Perkin-Elmer 1420 spectrometer. ${ }^{1} \mathrm{H}$ NMR and ${ }^{13} \mathrm{C}$ NMR spectra were recorded with $400 \mathrm{MHz}$ ARX 400 Brucker spectrometers. Chemical shifts are given in ppm, referenced to the residual proton resonances of the solvents. Coupling constants $(J)$ are given in Hertz $(\mathrm{Hz})$. The terms $\mathrm{m}, \mathrm{s}, \mathrm{d}$, t, q, quint indicate a multiplet, singlet, doublet, triplet, quadruplet and quintet respectively. The term br indicates that the signal is broad.

2-(1,3-Dioxa-2,2-dimethyl-cyclohex-5-yliden)-cyclohexanol (3a): DABCO (220 $\mathrm{mg}, 1.962 \mathrm{mmol}, 1.5 \mathrm{eq}$.) was added to a solution of $1 \mathrm{a}(315.3 \mathrm{mg}, 1.308 \mathrm{mmol}, 1$ eq.) in 1,2-dichlorobenzene (2 ml). The resultant mixture was heated at reflux for $3 \mathrm{~h}$. The crude product was purified by flash chromatography (PE/EA 8:2) to give $198 \mathrm{mg}$ $(72 \%)$ of the alcohol $3 \mathrm{a}$ as a colourless oil. $-{ }^{1} \mathrm{H}$ NMR: $\delta=4.52(\mathrm{~d}, \mathrm{~J}=3.2 \mathrm{~Hz}, 1 \mathrm{H}$, $\mathrm{CHO}) ; 4.42\left(\mathrm{~d}, \mathrm{~J}=3.5 \mathrm{~Hz}, 2 \mathrm{H}, \mathrm{CH}_{2}\right) ; 4.34\left(\mathrm{~s}, 2 \mathrm{H}, \mathrm{CH}_{2}\right) ; 2.3-2.1\left(\mathrm{~m}, 2 \mathrm{H}, \mathrm{CH}_{2}\right) ; 2.1-1.6$ $\left(\mathrm{m}, 4 \mathrm{H}, 2 \mathrm{CH}_{2}\right) ; 1.40\left(\mathrm{~s}, 3 \mathrm{H}, \mathrm{CH}_{3}\right) ; 1.38\left(\mathrm{~s}, 3 \mathrm{H}, \mathrm{CH}_{3}\right) ; 1.6-1.2\left(\mathrm{~m}, 2 \mathrm{H}, \mathrm{CH}_{2}\right) .-{ }^{13} \mathrm{C}$ NMR: $\delta=132.2$ (Cq.); 127.9 (Cq.); 99.8 (Cq.); $65.4(\mathrm{CHO}) ; 60.1\left(\mathrm{CH}_{2}\right) ; 59.3\left(\mathrm{CH}_{2}\right) ; 33.9$ $\left(\mathrm{CH}_{3}\right) ; 26.8\left(\mathrm{CH}_{2}\right) ; 24.4\left(\mathrm{CH}_{2}\right) ; 24.2\left(\mathrm{CH}_{2}\right) ; 23.9\left(\mathrm{CH}_{2}\right) ; 21.2\left(\mathrm{CH}_{3}\right)$. - IR (neat): $v\left(\mathrm{~cm}^{-}\right.$ $\left.{ }^{1}\right)=3442(\mathrm{C}-\mathrm{OH}) .-\mathrm{Mp} 96{ }^{\circ} \mathrm{C}$ (1,2-dichloromethane, heptane). - Anal. Calcd. for $\mathrm{C}_{12} \mathrm{H}_{20} \mathrm{O}_{3} \mathrm{C}$ (67.89), $\mathrm{H}$ (9.5); found C (67.69), $\mathrm{H}$ (9.41).

4-(2-Hydroxy-cyclohexylidene)-heptanedinitrile (3b): DABCO (68 mg, 0.607 $\mathrm{mmol}, 1.5 \mathrm{eq}$.) was added to a solution of $\mathbf{1 b}(100 \mathrm{mg}, 0.405 \mathrm{mmol}, 1 \mathrm{eq}$.) in $1,2-$ dichlorobenzene $(1.6 \mathrm{ml})$. The resultant mixture was heated at reflux for $30 \mathrm{~min}$. The crude product was purified by flash chromatography (PE/EA 6:4 to 5:5) to give $80 \mathrm{mg}$ $(91 \%)$ of the alcohol $\mathbf{3 b}$ as a colourless oil. ${ }^{1} \mathrm{H}$ NMR: $\delta=4.72$ (s br, $\left.1 \mathrm{H}, \mathrm{CHO}\right) ; 2.61$ $2.21\left(\mathrm{~m}, 10 \mathrm{H}, 5 \mathrm{CH}_{2}\right) ; 2.02-1.25\left(\mathrm{~m}, 6 \mathrm{H}, 3 \mathrm{CH}_{2}\right) .-{ }^{13} \mathrm{C} \mathrm{NMR}: \delta=142.9$ (Cq.); 124.8 
(Cq.); 120.0 (Cq.); 119.2 (Cq.); $65.8(\mathrm{CHO}) ; 34.1\left(\mathrm{CH}_{2}\right) ; 27.7\left(\mathrm{CH}_{2}\right) ; 27.2\left(\mathrm{CH}_{2}\right) ; 26.8$ $\left(\mathrm{CH}_{2}\right) ; 25.5\left(\mathrm{CH}_{2}\right) ; 19.3\left(\mathrm{CH}_{2}\right) ; 17.3\left(\mathrm{CH}_{2}\right) ; 16.8\left(\mathrm{CH}_{2}\right)$. - IR (neat): $v\left(\mathrm{~cm}^{-1}\right)=3559(\mathrm{C}-$ $\mathrm{OH}) ; 2246(\mathrm{CN}) .-\mathrm{MS}\left(\mathrm{Cl}, \mathrm{NH}_{3}\right) \mathrm{m} / \mathrm{z}=\left[\mathrm{MH}+\mathrm{NH}_{3}\right]^{+}=236 ;[\mathrm{MH}]^{+}=219 ;\left[\mathrm{MH}-\mathrm{H}_{2} \mathrm{O}\right]^{+}=$ 201.

\section{2-[3-Benzenesulfonyl-1-(2-benzenesulfonyl-ethyl)-propylidene]-cyclohexanol}

(3c): DABCO (29 mg, $0.258 \mathrm{mmol}, 1.5$ eq.) was added to a solution of 1c (82 mg, $0.172 \mathrm{mmol}, 1$ eq.) in 1,2-dichlorobenzene (1.6 ml). The resultant mixture was heated at reflux for $40 \mathrm{~min}$. The crude product was purified by flash chromatography (PE/EA $6: 4$ to $5: 5)$ to give $58 \mathrm{mg}(75 \%)$ of the alcohol $3 \mathrm{c}$ as a yellow solid $\left(\mathrm{mp} 114-116^{\circ} \mathrm{C}\right)$. ${ }^{1} \mathrm{H}$ NMR: $\delta=7.88(\mathrm{~d}, \mathrm{~J}=7.6 \mathrm{~Hz}, 2 \mathrm{H}, \mathrm{CH}=) ; 7.68(\mathrm{t}, \mathrm{J}=7.6 \mathrm{~Hz}, 1 \mathrm{H}, \mathrm{CH}=) ; 7.58(\mathrm{t}, \mathrm{J}=$ 7.6Hz, $2 \mathrm{H}, 2 \mathrm{CH}=) ; 4.61$ (s br, $1 \mathrm{H}, \mathrm{CHO}) ; 3.22-2.97\left(\mathrm{~m}, 4 \mathrm{H}, 2 \mathrm{CH}_{2}\right) ; 2.71-2.29(\mathrm{~m}, 4 \mathrm{H}$, $\left.2 \mathrm{CH}_{2}\right) ; 2.17-1.03\left(\mathrm{~m}, 8 \mathrm{H}, 4 \mathrm{CH}_{2}\right) .-{ }^{13} \mathrm{C}$ NMR: $\delta=141.1$ (Cq.); 138.9 (Cq.); 138.7 (Cq.) ; $133.9(\mathrm{CH}=) ; 129.4(2 \mathrm{CH}=) ; 127.5(2 \mathrm{CH}=) ; 123.9$ (Cq. ; 65.7 (CHO); 54.9 $\left(\mathrm{CH}_{2}\right) ; 54.8\left(\mathrm{CH}_{2}\right) ; 34.1\left(\mathrm{CH}_{2}\right) ; 27.2\left(\mathrm{CH}_{2}\right) ; 25.31\left(\mathrm{CH}_{2}\right) ; 24.6\left(\mathrm{CH}_{2}\right) ; 24.2\left(\mathrm{CH}_{2}\right) ; 19.7$ $\left(\mathrm{CH}_{2}\right)$. - IR (neat): $v\left(\mathrm{~cm}^{-1}\right)=3547(\mathrm{C}-\mathrm{OH}) ; 1321\left(\mathrm{C}-\mathrm{SO}_{2}\right) ; 1151\left(\mathrm{C}-\mathrm{SO}_{2}\right)-\mathrm{MS}(\mathrm{Cl}$, $\left.\mathrm{NH}_{3}\right) \mathrm{m} / \mathrm{z}=\left[\mathrm{MH}+\mathrm{NH}_{3}\right]^{+}=466 ;[\mathrm{MH}]^{+}=449 ;\left[\mathrm{MH}-\mathrm{H}_{2} \mathrm{O}\right]^{+}=431$.

Acetic acid 1-nitromethyl-3-phenyl-propyl ester (6): A solution of dihydrocinnamaldehyde $(1.96 \mathrm{ml}, 0.015 \mathrm{~mol}, 1$ eq.), nitroethane $(5.39 \mathrm{ml}, 0.075 \mathrm{~mol}$, 5 eq.) and $\mathrm{NEt}_{3}(0.14 \mathrm{ml})$ was stirred for $1 \mathrm{~h}$ at room temp. The reaction mixture was then poured into solution of citric acid, and extracted with 1,2-dichloromethane. The extracts were washed with water and dried with anhydrous $\mathrm{Na}_{2} \mathrm{SO}_{4}$, filtered and concentrated. The residue was dissolved in 1,2-dichloromethane (30 ml). $\mathrm{Ac}_{2} \mathrm{O}(4.25$ $\mathrm{ml}, 0.045 \mathrm{~mol}, 3$ eq.) and DMAP (183 mg, $1.5 \mathrm{mmol}, 10 \%$ ) were added at $0^{\circ} \mathrm{C}$ and the reaction mixture was stirred for 2 hours. $\mathrm{MeOH}$ was then added and the reaction mixture was washed with sat. aq. $\mathrm{NaHCO}_{3}$ and extracted with 1,2-dichloromethane. The organic layer was washed with brine, dried with anhydrous $\mathrm{Na}_{2} \mathrm{SO}_{4}$, filtered, and concentrated. The crude product was purified by flash chromatography (PE/EA 9:1) to give $2.6 \mathrm{~g}$ of $6(70 \%, 2$ steps) as a colourless oil and as a mixture of 2 diastereomers in a 1:1 ratio. ${ }^{1} \mathrm{H}$ NMR: $\delta=7.36-7.20$ (massif, $\left.5 \mathrm{H}\right) ; 5.38(\mathrm{~m}, 1 \mathrm{H}$, $\mathrm{CHO}) ; 4.76(\mathrm{~m}, 1 \mathrm{H}, \mathrm{CHN}) ; 2.71\left(\mathrm{~m}, 2 \mathrm{H}, \mathrm{CH}_{2}\right) ; 2.12$ and $2.09\left(\mathrm{~s}, 3 \mathrm{H}, \mathrm{CH}_{3}\right) ; 1.99(\mathrm{~m}$, $\left.2 \mathrm{H}, \mathrm{CH}_{2}\right) ; 1.58$ and $1.56\left(\mathrm{~d}, \mathrm{~J}=2.2 \mathrm{~Hz}, 3 \mathrm{H}, \mathrm{CH}_{3}\right) .-{ }^{13} \mathrm{C}$ NMR: $\delta=170.0$ and 169.4 (CO); 140.3 and 140.2 (Cq.); $128.5(2 \mathrm{CH}=) ; 128.2(2 \mathrm{CH}=) ; 126.3(\mathrm{CH}=) ; 84.3$ and 83.7 $(\mathrm{CHN}) ; 72.9$ and $72.7(\mathrm{CHO}) ; 32.1$ and $31.7\left(\mathrm{CH}_{2}\right)$; 31.6 and $31.0\left(\mathrm{CH}_{2}\right) ; 22.1$ and $20.6\left(\mathrm{CH}_{3}\right) ; 15.4$ and $13.5\left(\mathrm{CH}_{3}\right)$. - IR (neat): $v\left(\mathrm{~cm}^{-1}\right)=1751(\mathrm{C}=\mathrm{O}) ; 1559.3$ (C$\left.\mathrm{NO}_{2}\right) ; 1225.3(\mathrm{C}-\mathrm{O}) .-\mathrm{MS}\left(\mathrm{Cl}, \mathrm{NH}_{3}\right) \mathrm{m} / \mathrm{z}=\left[\mathrm{MH}+\mathrm{NH}_{3}\right]^{+}=369 ;[\mathrm{MH}]^{+}=252$.

\section{Acetic acid (E)-2-acetoxymethyl-2-nitro-5-phenyl-pent-3-enyl ester (1d):}

Formaldehyde $37 \%$ solution $\left(6.73 \mathrm{ml}\right.$ of a $37 \%$ solution in water) and $\mathrm{Et}_{3} \mathrm{~N}(6.215$ $\mathrm{mmol}, 1.2 \mathrm{eq}$.) were added to a solution of $6(1.3 \mathrm{~g}, 5.179 \mathrm{mmol}, 1 \mathrm{eq}$.) in acetonitrile $(15.5 \mathrm{ml})$. The resultant mixture was heated at reflux for $1 \mathrm{~h}$. The reaction mixture was then poured into a aqueous solution of citric acid, and extracted with 1,2dichloromethane. The extracts were washed with brine and dried with anhydrous 
dichloromethane (8.4 ml). $\mathrm{Ac}_{2} \mathrm{O}(1.47 \mathrm{ml}, 15.537 \mathrm{mmol}, 3 \mathrm{eq}$.$) and DMAP (63 mg,$ $0.518 \mathrm{mmol}, 10 \%$ ) were added at $0^{\circ} \mathrm{C}$ and the reaction mixture was stirred for 2 hours. $\mathrm{MeOH}$ was then added and the reaction mixture was washed with sat. aq. $\mathrm{NaHCO}_{3}$ and extracted with 1,2-dichloromethane. The organic layer was washed with brine, dried with anhydrous $\mathrm{Na}_{2} \mathrm{SO}_{4}$, filtered, and concentrated. The crude product was purified by flash chromatography (PE/EA 9:1) to give $905 \mathrm{mg}$ of $\mathbf{1 d}(66 \%, 2$ steps) as a colourless oil. $-{ }^{1} \mathrm{H}$ NMR: $\delta=7.34-7.14(\mathrm{~m}, 5 \mathrm{H}) ; 6.07(\mathrm{dt}, \mathrm{J}=15.6 \mathrm{~Hz}, \mathrm{~J}=$ $6.8 \mathrm{~Hz}, 1 \mathrm{H}, \mathrm{CH}=) ; 5.81(\mathrm{dt}, \mathrm{J}=15.6 \mathrm{~Hz}, \mathrm{~J}=1.6 \mathrm{~Hz}, 1 \mathrm{H}, \mathrm{CH}=) ; 4.59\left(\mathrm{~d}, \mathrm{~J}_{\mathrm{AB}}=11.8 \mathrm{~Hz}\right.$, $\left.1 \mathrm{H}, \mathrm{CH}_{2}\right) ; 4.34\left(\mathrm{~d}, \mathrm{~J}_{\mathrm{AB}}=11.8 \mathrm{~Hz}, 1 \mathrm{H}, \mathrm{CH}_{2}\right) ; 3.45\left(\mathrm{~d}, \mathrm{~J}=6.8 \mathrm{~Hz}, 2 \mathrm{H}, \mathrm{CH}_{2}\right) ; 2.07(\mathrm{~s}, 3 \mathrm{H}$, $\left.\mathrm{CH}_{3}\right), 1.74\left(\mathrm{~s}, 3 \mathrm{H}, \mathrm{CH}_{3}\right) .-{ }^{13} \mathrm{C}$ NMR: $\delta=170.2(\mathrm{CO}) ; 138.3(\mathrm{Cq}) .134.9(\mathrm{CH}=) ; 128.6$ $(2 \mathrm{CH}=) ; 128.5(2 \mathrm{CH}=) ; 126.9(\mathrm{CH}=) ; 126.5(\mathrm{CH}=) ; 88.9(\mathrm{Cq}.) ; 67.6\left(\mathrm{CH}_{2}\right) ; 38.6\left(\mathrm{CH}_{2}\right)$; $20.5\left(\mathrm{CH}_{3}\right) ; 19.9\left(\mathrm{CH}_{3}\right)$. - IR (neat): $v\left(\mathrm{~cm}^{-1}\right)=1754.2(\mathrm{C}=\mathrm{O}) ; 1555\left(\mathrm{C}-\mathrm{NO}_{2}\right) ; 1227(\mathrm{C}-$ O).- $\mathrm{MS}\left(\mathrm{Cl}, \mathrm{NH}_{3}\right) \mathrm{m} / \mathrm{z}=\left[\mathrm{MH}+\mathrm{NH}_{3}\right]^{+}=281 ;[\mathrm{MH}]^{+}=264 ;\left[\mathrm{MH}-\mathrm{NO}_{2}\right]^{+}=218$.

Acetic acid 2-acetoxymethyl-4-hydroxy-5-phenyl-pent-2-enyl ester (3d): DABCO $(64 \mathrm{mg}, 0.570 \mathrm{mmol}, 1.5 \mathrm{eq}$.) was added to a solution of $1 \mathbf{d}(100 \mathrm{mg}, 0.380 \mathrm{mmol}, 1$ eq.) in 1,2-dichlorobenzene $(1.6 \mathrm{ml})$. The resultant mixture was heated at reflux for 30 min. The crude product was purified by flash chromatography (PE/EA 6:4 to 5:5) to give $77 \mathrm{mg}(87 \%)$ of the alcohol $3 \mathbf{d}$ as a colourless oil and as a mixture of diastereomers in a $E: Z$ ratio of 2:1. $-{ }^{1} \mathrm{H}$ NMR: $\delta=7.32-7.20(\mathrm{~m}, 5 \mathrm{H}) ; 5.51(\mathrm{dq}, \mathrm{J}=8.4$ $\mathrm{Hz}, \mathrm{J}=1.4 \mathrm{~Hz}, 0.67 \mathrm{H}, \mathrm{CH}=) ; 5.48$ (dt, J=8.8 Hz, J=0.6 Hz, 0.33H, CH=); 4.62 (m, $1 \mathrm{H}, \mathrm{CHO}) ; 4.65\left(\mathrm{~d}, \mathrm{~J}_{\mathrm{AB}}=12.4 \mathrm{~Hz}, 0.33 \mathrm{H}, \mathrm{CH}_{2} \mathrm{O}\right) ; 4.44\left(\mathrm{~s}, 1.34 \mathrm{H}, \mathrm{CH}_{2} \mathrm{O}\right) ; 4.29\left(\mathrm{~d}, \mathrm{~J}_{\mathrm{AB}}\right.$ $\left.=12.4 \mathrm{~Hz}, 0.33 \mathrm{H}, \mathrm{CH}_{2} \mathrm{O}\right) ; 2.82\left(\mathrm{~m}, 2 \mathrm{H}, \mathrm{CH}_{2}\right) ; 2.08$ and $2.03\left(\mathrm{~s}, 0.99 \mathrm{H}, \mathrm{CH}_{3}\right) ; 1.75$ and $1.53\left(\mathrm{~s}, 3 \mathrm{H}, \mathrm{CH}_{3}\right) .-{ }^{13} \mathrm{C}$ NMR: $\delta=171.1$ and $170.7(\mathrm{CO}) ; 137.7$ and 137.5 (Cq.); 132.7 and 132.4 (Cq.); 129.8 and $129.50(\mathrm{CH}=) ; 129.55(2 \mathrm{CH}=) ; 128.39(2 \mathrm{CH}=)$; 126.48 and $126.42(\mathrm{CH}=) ; 69.19$ and $68.6(\mathrm{CH}) ; 68.8$ and $63.1\left(\mathrm{CH}_{2}\right) ; 43.8\left(\mathrm{CH}_{2}\right)$; 21.2 and $20.9\left(\mathrm{CH}_{3}\right) ; 14.1\left(\mathrm{CH}_{3}\right)$. - IR (neat): $v\left(\mathrm{~cm}^{-1}\right)=3496(\mathrm{C}-\mathrm{OH}) ; 1740(\mathrm{C}=\mathrm{O})$; 1235 (C-O). $-\mathrm{MS}\left(\mathrm{Cl}, \mathrm{NH}_{3}\right) \mathrm{m} / \mathrm{z}=\left[\mathrm{MH}+\mathrm{NH}_{3}\right]^{+}=252 ;[\mathrm{MH}]^{+}=235 ;\left[\mathrm{MH}-\mathrm{H}_{2} \mathrm{O}\right]^{+}=217$.

4-[2-Hydroxy-cyclohex-(E)-ylidene]-1,3-diphenyl-butan-1-one (3f): DABCO (48 $\mathrm{mg}, 0.430 \mathrm{mmol}, 1.5 \mathrm{eq}$.) was added to a solution of $1 \mathrm{f}(100 \mathrm{mg}, 0.287 \mathrm{mmol}, 1 \mathrm{eq}$.) in 1,2-dichlorobenzene $(1.6 \mathrm{ml})$. The resultant mixture was heated at reflux for $1 \mathrm{~h}$. The crude product was purified by flash chromatography (PE/EA 6:4 to 5:5) to give $62 \mathrm{mg}(68 \%)$ of the alcohol $3 f$ as a colourless oil. $-{ }^{1} \mathrm{H}$ NMR: $\delta=7.92(\mathrm{~d}, \mathrm{~J}=7.2 \mathrm{~Hz}$, $2 \mathrm{H}, 2 \mathrm{CH}=) ; 7.55(\mathrm{t}, \mathrm{J}=7.2 \mathrm{~Hz}, 1 \mathrm{H}, \mathrm{CH}=) ; 7.45(\mathrm{t}, \mathrm{J}=7.2 \mathrm{~Hz}, 2 \mathrm{H}, 2 \mathrm{CH}=) ; 7.28(\mathrm{~m}$, $4 \mathrm{H}, 4 \mathrm{CH}=) ; 7.19(\mathrm{~m}, 1 \mathrm{H}, \mathrm{CH}=) ; 5.55(\mathrm{~d}, \mathrm{~J}=10 \mathrm{~Hz}, 1 \mathrm{H}, \mathrm{CH}=) ; 4.36(\mathrm{~m}, 1 \mathrm{H} \mathrm{CH}-\mathrm{Ph}) ; 3.98$ (s br, $1 \mathrm{H}, \mathrm{CHO}) ; 3.36\left(\mathrm{~m}, 2 \mathrm{H}, \mathrm{CH}_{2}\right) ; 2.55$ and $1.93\left(\mathrm{~m}, 2 \mathrm{H}, \mathrm{CH}_{2}\right) ; 1.77\left(\mathrm{~m}, 2 \mathrm{H}, \mathrm{CH}_{2}\right)$; $1.41\left(\mathrm{~m}, 4 \mathrm{H}, 2 \mathrm{CH}_{2}\right) .-{ }^{13} \mathrm{C}$ NMR: $\delta=198.7$ (CO); 144.7 (Cq.); 141.9 (Cq.); 137.3 (Cq.); $133.0(\mathrm{CH}=) ; 128.7(2 \mathrm{CH}=) ; 128.6(2 \mathrm{CH}=) ; 128.2(2 \mathrm{CH}=) ; 127.3(2 \mathrm{CH}=) ; 126.3$ $(\mathrm{CH}=) ; 123.1(\mathrm{CH}=) ; 73.4(\mathrm{CHO}) ; 45.8\left(\mathrm{CH}_{2}\right) ; 39.0(\mathrm{CH}) ; 36.3\left(\mathrm{CH}_{2}\right) ; 27.0\left(\mathrm{CH}_{2}\right) ; 26.8$ $\left(\mathrm{CH}_{2}\right) ; 23.2\left(\mathrm{CH}_{2}\right)$. - IR (neat): $v\left(\mathrm{~cm}^{-1}\right)=3464(\mathrm{C}-\mathrm{OH}) ; 1691(\mathrm{C}=\mathrm{O}) .-\mathrm{MS}\left(\mathrm{Cl}, \mathrm{NH}_{3}\right)$ $\mathrm{m} / \mathrm{z}=\left[\mathrm{MH}+\mathrm{NH}_{3}\right]^{+}=338 ;[\mathrm{MH}]^{+}=321 ;\left[\mathrm{MH}-\mathrm{H}_{2} \mathrm{O}\right]^{+}=303$. 
Acetic acid 1-nitro-cyclohex-2-enylmethyl ester (1g): (i) A solution of 1nitrocyclohexene $(500 \mathrm{mg}, 3.932 \mathrm{mmol}, 1$ eq.), formaldehyde $(0.5 \mathrm{ml}$ of a $37 \%$ solution in water) and isopropyldiethylamine $(0.068 \mathrm{ml}, 0.393 \mathrm{mmol}, 0.1 \mathrm{eq}$.) in acetonitrile $(3.9 \mathrm{ml})$ was stirred at room temp. for $3 \mathrm{~h}$. The reaction mixture was poured into water containing $1 \mathrm{~N}$ hydrochloric acid, and extracted with AcOEt. The organic extracts were washed with brine and dried with anhydrous $\mathrm{Na}_{2} \mathrm{SO}_{4}$, filtered and concentrated. The crude product was purified by flash chromatography (PE/EA $8: 2)$ to give $283 \mathrm{mg} \mathrm{(46 \% )}$ of the alcohol.

(ii) $\mathrm{Ac}_{2} \mathrm{O}$ (0.30 ml, $3.210 \mathrm{mmol}, 3$ eq.) and DMAP (13mg, $\left.0.107 \mathrm{mmol}, 10 \%\right)$ were added at $0^{\circ} \mathrm{C}$ to a solution of the alcohol $(168 \mathrm{mg}, 1.070 \mathrm{mmol}, 1$ eq.) in $1,2-$ dichloromethane $(2.2 \mathrm{ml})$ and the reaction mixture was stirred for 2 hours. $\mathrm{MeOH}$ was then added and the reaction mixture was quenched with sat. aq. $\mathrm{NaHCO}_{3}$. The organic layer was washed with brine, dried with anhydrous $\mathrm{Na}_{2} \mathrm{SO}_{4}$, filtered, and concentrated to give a crude oil which was purified using column chromatography (PE/EA $8: 2)$ to give $173 \mathrm{mg}(81 \%)$ of $1 \mathrm{~g}$ as a colourless oil. $-{ }^{1} \mathrm{H}$ NMR: $\delta=6.21(\mathrm{~m}$, $1 \mathrm{H}, \mathrm{CH}=) ; 5.91(\mathrm{~d}, \mathrm{~J}=10.6 \mathrm{~Hz}, 1 \mathrm{H}, \mathrm{CH}=) ; 4.48\left(\mathrm{~d}, \mathrm{~J}_{\mathrm{AB}}=12 \mathrm{~Hz}, 1 \mathrm{H}, \mathrm{CH}_{2} \mathrm{O}\right) ; 4.37(\mathrm{~d}$, $\left.\mathrm{J}_{\mathrm{AB}}=12 \mathrm{~Hz}, 1 \mathrm{H}, \mathrm{CH}_{2} \mathrm{O}\right) ; 2.53\left(\mathrm{~m}, 1 \mathrm{H}, \mathrm{CH}_{2}\right) ; 2.19\left(\mathrm{~m}, 1 \mathrm{H}, \mathrm{CH}_{2}\right) ; 2.07\left(\mathrm{~s}, 3 \mathrm{H}, \mathrm{CH}_{3}\right) ; 1.73$ $\left(\mathrm{m}, 4 \mathrm{H}, 2 \mathrm{CH}_{2}\right) .{ }^{13} \mathrm{C}$ NMR: $\delta=170.2(\mathrm{CO}) ; 136.04(\mathrm{CH}=) ; 121.8(\mathrm{CH}=) ; 87.3(\mathrm{Cq}$.); $68.2\left(\mathrm{CH}_{2} \mathrm{O}\right) ; 29.2\left(\mathrm{CH}_{2}\right) ; 24.8\left(\mathrm{CH}_{2}\right) ; 20.6\left(\mathrm{CH}_{3}\right) ; 18.3\left(\mathrm{CH}_{2}\right)$. I IR (neat): $v\left(\mathrm{~cm}^{-1}\right)=$ $1754(\mathrm{C}=\mathrm{O}) ; 1549\left(\mathrm{C}-\mathrm{NO}_{2}\right) ; 1221(\mathrm{C}-\mathrm{O}) .-\mathrm{MS}\left(\mathrm{Cl}, \mathrm{NH}_{3}\right) \mathrm{m} / \mathrm{z}=\left[\mathrm{MH}+\mathrm{NH}_{3}\right]^{+}=217$; $[\mathrm{MH}]^{+}=200 ;\left[\mathrm{MH}-\mathrm{NO}_{2}\right]^{+}=154$

Acetic acid 3-hydroxy-cyclohex-1-enylmethyl ester (3g): DABCO (62 mg, 0.550 $\mathrm{mmol}, 1.5 \mathrm{eq}$.) was added to a solution of $1 \mathrm{~g}(62 \mathrm{mg}, 0.550 \mathrm{mmol}, 1.5 \mathrm{eq}$.) in $1,2-$ dichlorobenzene $(1.6 \mathrm{ml})$. The resultant mixture was heated at reflux for $30 \mathrm{~min}$. The crude product was purified by flash chromatography (PE/EA 6:4 to 5:5) to give $45 \mathrm{mg}$ $(72 \%)$ of the alcohol $\mathbf{3 g}$ as a colourless oil. $-{ }^{1} \mathrm{H}$ NMR: $\delta=5.78(\mathrm{~s} \mathrm{br}, 1 \mathrm{H}, \mathrm{CH}=) ; 4.47$ (s, 2H, $\left.\mathrm{CH}_{2} \mathrm{O}\right) ; 4.25$ (s br, $\left.1 \mathrm{H}, \mathrm{CH}-\mathrm{OH}\right) ; 2.08\left(\mathrm{~s}, 3 \mathrm{H}, \mathrm{CH}_{3}\right) ; 2.01-1.58\left(\mathrm{M}, 6 \mathrm{H}, 3 \mathrm{CH}_{2}\right)$.${ }^{13} \mathrm{C}$ NMR: $\delta=170.9(\mathrm{CO}) ; 136.5(\mathrm{Cq})$ ); $127.2(\mathrm{CH}=) ; 67.5\left(\mathrm{CH}_{2} \mathrm{O}\right) ; 65.4(\mathrm{CH}-\mathrm{OH})$; $31.6\left(\mathrm{CH}_{2}\right) ; 25.7\left(\mathrm{CH}_{2}\right) ; 20.9\left(\mathrm{CH}_{3}\right) ; 18.7\left(\mathrm{CH}_{2}\right)$. IR (neat): $v\left(\mathrm{~cm}^{-1}\right)=3467(\mathrm{C}-\mathrm{OH})$; $1744(\mathrm{C}=\mathrm{O}) ; 1226(\mathrm{C}-\mathrm{O}) .-\mathrm{MS}\left(\mathrm{Cl}, \mathrm{NH}_{3}\right) \mathrm{m} / \mathrm{z}=\left[\mathrm{MH}+\mathrm{NH}_{3}\right]^{+}=188 ;[\mathrm{MH}]^{+}=171 ;[\mathrm{MH}-$ $\left.\mathrm{H}_{2} \mathrm{O}\right]^{+}=153$.

4-(1-Nitro-cyclohex-2-enyl)-butan-2-one (1h): A solution of 1-nitrocyclohexene 6 (500 mg, $3.932 \mathrm{mmol}, 1$ eq.) , methyl vinyl ketone $(0.64 \mathrm{ml}, 7.864 \mathrm{mmol}$, 2eq.) and DBU $(0.06 \mathrm{ml}, 0.393 \mathrm{mmol}, 0.1$ eq.) in acetonitrile $(6 \mathrm{ml})$ was stirred at room temp. for $4 \mathrm{~h}$. The acetonitrile was evaporated under reduced pressure. The crude product was purified by flash chromatography (PE/EA 8:2 to 6:4) to give $728 \mathrm{mg}(94 \%)$ of $\mathbf{1 h}$ as a colourless oil. $-{ }^{1} \mathrm{H}$ NMR: $\delta=6.10$ (dt, $\mathrm{J}=10.20 \mathrm{~Hz}, \mathrm{~J}=3.2 \mathrm{~Hz}, 1 \mathrm{H}, \mathrm{CH}=$ ); 5.88 (d, $\mathrm{J}=10.20 ; 1 \mathrm{H}, \mathrm{CH}=) ; 2.51\left(\mathrm{~m}, 3 \mathrm{H}, 2 \mathrm{CH}_{2}\right) ; 2.23-2.03\left(\mathrm{~m}, 3 \mathrm{H}, 2 \mathrm{CH}_{2}\right) ; 2.16(\mathrm{~s}, 3 \mathrm{H}$, $\left.\mathrm{CH}_{3}\right) ; 2.05\left(\mathrm{~m}, 2 \mathrm{H}, \mathrm{CH}_{2}\right) ; 1.79-1.52\left(\mathrm{~m}, 4 \mathrm{H}, 2 \mathrm{CH}_{2}\right) .-{ }^{13} \mathrm{C} \mathrm{NMR:} \delta=206.3(\mathrm{CO})$; $134.1(\mathrm{CH}=) ; 124.96(\mathrm{CH}=) ; 88.3(\mathrm{Cq}.) ; 37.5\left(\mathrm{CH}_{2}\right) ; 33.89\left(\mathrm{CH}_{2}\right) ; 31.8\left(\mathrm{CH}_{2}\right) ; 29.9$ $\left(\mathrm{CH}_{3}\right) ; 24.7\left(\mathrm{CH}_{2}\right) ; 18.2\left(\mathrm{CH}_{2}\right)$. - IR (neat): $v\left(\mathrm{~cm}^{-1}\right)=1723(\mathrm{C}=\mathrm{O}) ; 1538\left(\mathrm{C}-\mathrm{NO}_{2}\right)$. $\mathrm{MS}\left(\mathrm{Cl}, \mathrm{NH}_{3}\right) \mathrm{m} / \mathrm{z}=\left[\mathrm{MH}+\mathrm{NH}_{3}\right]^{+}=215 ;[\mathrm{MH}]^{+}=198$. 
4-(3-Hydroxy-cyclohex-1-enyl)-butan-2-one (3h): DABCO (85 mg, $0.761 \mathrm{mmol}, 1.5$ eq.) was added to a solution of $1 \mathrm{~h}(100 \mathrm{mg}, 0.508 \mathrm{mmol}, 1 \mathrm{eq}$.$) in 1,2-$

dichlorobenzene $(1.6 \mathrm{ml})$. The resultant mixture was heated at reflux for $30 \mathrm{~min}$. The crude product was purified by flash chromatography (PE/EA 6:4 to 5:5) to give $62 \mathrm{mg}$ (73\%) of the alcohol $3 \mathrm{~h}$ as a colourless oil. $-{ }^{1} \mathrm{H}$ NMR: $\delta=5.47(\mathrm{~s} \mathrm{br}, 1 \mathrm{H}, \mathrm{CH}=) ; 4.19$ (s br, $1 \mathrm{H}, \mathrm{CHO}) ; 2.57$ (t, J=7.63, 2H, $\left.\mathrm{CH}_{2}\right) ; 2.25$ (t, J=7.63, 2H, $\left.\mathrm{CH}_{2}\right) ; 2.16(\mathrm{~s}, 3 \mathrm{H}$, $\left.\mathrm{CH}_{3}\right) ; 1.95-1.55\left(\mathrm{~m}, 6 \mathrm{H}, 3 \mathrm{CH}_{2}\right) .-{ }^{13} \mathrm{C}$ NMR: $\delta=208.4(\mathrm{CO}) ; 140.7$ (Cq.); 124.0 $(\mathrm{CH}=) ; 65.7(\mathrm{CH}) ; 41.45\left(\mathrm{CH}_{2}\right) ; 31.7\left(\mathrm{CH}_{2}\right) ; 31.00\left(\mathrm{CH}_{2}\right) ; 29.9\left(\mathrm{CH}_{3}\right) ; 28.6\left(\mathrm{CH}_{2}\right) ; 19.0$ $\left(\mathrm{CH}_{2}\right) .-\mathrm{IR}$ (neat): $v\left(\mathrm{~cm}^{-1}\right)=3425(\mathrm{C}-\mathrm{OH}) ; 1720(\mathrm{C}=\mathrm{O}) .-\mathrm{MS}\left(\mathrm{Cl}, \mathrm{NH}_{3}\right) \mathrm{m} / \mathrm{z}=$ $\left[\mathrm{MH}+\mathrm{NH}_{3}\right]^{+}=186 ;[\mathrm{MH}]^{+}=169 ;\left[\mathrm{MH}-\mathrm{H}_{2} \mathrm{O}\right]^{+}=151$

3-(1-Nitro-cyclohex-2-enyl)-propionic acid methyl ester (1i): A solution of 1nitrocyclohexene $6(500 \mathrm{mg}, 3.932 \mathrm{mmol}, 1$ eq. $)$, methyl acrylate $(0.71 \mathrm{ml}, 7.864$ $\mathrm{mmol}$, 2eq.) and $\mathrm{DBU}(0.06 \mathrm{ml}, 0.393 \mathrm{mmol}, 0.1$ eq. $)$ in acetonitrile $(6 \mathrm{ml})$ was stirred at room temp. overnight. The acetonitrile was evaporated under reduced pressure. The crude product was purified by flash chromatography (PE/EA 8:2 to 6:4) to give $586 \mathrm{mg}(70 \%)$ of $1 \mathrm{i}$ as a colourless oil. $-{ }^{1} \mathrm{H}$ NMR: $\delta=6.11(\mathrm{~m}, 1 \mathrm{H}, \mathrm{CH}=) ; 5.89(\mathrm{~m}$, $1 \mathrm{H}, \mathrm{CH}=)$; 3.68(s, 3H, $\left.\mathrm{CH}_{3}\right) ; 2.56-1.99\left(\mathrm{~m}, 6 \mathrm{H}, 3 \mathrm{CH}_{2}\right) ; 1.78-1.55\left(\mathrm{~m}, 4 \mathrm{H}, 2 \mathrm{CH}_{2}\right) .-{ }^{13} \mathrm{C}$ NMR: $\delta=172.5(\mathrm{CO}) ; 134.3(\mathrm{CH}=) ; 124.8(\mathrm{CH}=) ; 88.2(\mathrm{Cq}.) ; 51.9\left(\mathrm{CH}_{3}\right) ; 35.2\left(\mathrm{CH}_{2}\right)$; $31.5\left(\mathrm{CH}_{2}\right) ; 28.5\left(\mathrm{CH}_{2}\right) ; 24.7\left(\mathrm{CH}_{2}\right) ; 18.7\left(\mathrm{CH}_{2}\right)$ - - IR (neat): $v\left(\mathrm{~cm}^{-1}\right)=1743(\mathrm{C}=\mathrm{O})$; $1544\left(\mathrm{C}-\mathrm{NO}_{2}\right) ; 1175(\mathrm{C}-\mathrm{O}) .-\mathrm{MS}\left(\mathrm{Cl}, \mathrm{NH}_{3}\right) \mathrm{m} / \mathrm{z}=\left[\mathrm{MH}+\mathrm{NH}_{3}\right]^{+}=231 ;[\mathrm{MH}]^{+}=214$.

3-(3-Hydroxy-cyclohex-1-enyl)-propionic acid methyl ester (3i): DABCO (79 mg, $0.704 \mathrm{mmol}, 1.5 \mathrm{eq}$.) was added to a solution of $1 \mathrm{i}$ (100 $\mathrm{mg}, 0.469 \mathrm{mmol}, 1 \mathrm{eq}$.) in 1,2-dichlorobenzene $(1.6 \mathrm{ml})$. The resultant mixture was heated at reflux for $30 \mathrm{~min}$. The crude product was purified by flash chromatography (PE/EA 6:4 to 5:5) to give $76 \mathrm{mg}(88 \%)$ of the alcohol $3 \mathbf{i}$ as a colourless oil. $-{ }^{1} \mathrm{H}$ NMR: $\delta=5.46(\mathrm{~s} \mathrm{br}, 1 \mathrm{H}, \mathrm{CH}=)$; 4.15 (s br, $1 \mathrm{H}, \mathrm{CH}-\mathrm{OH}) ; 3.63\left(\mathrm{~s}, 3 \mathrm{H}, \mathrm{CH}_{3}\right) ; 2.41$ (t, J=8 Hz, $\left.2 \mathrm{H}, \mathrm{CH}_{2}\right) ; 2.26(\mathrm{t}, \mathrm{J}=8 \mathrm{~Hz}$, $\left.2 \mathrm{H}, \mathrm{CH}_{2}\right) ; 1.93-1.51\left(\mathrm{~m}, 6 \mathrm{H}, 3 \mathrm{CH}_{2}\right) .-{ }^{13} \mathrm{C} \mathrm{NMR:} \delta=173.7$ (CO); 140.3 (Cq.); 124.3 $(\mathrm{CH}=) ; 65.6(\mathrm{CH}-\mathrm{OH}) ; 51.6\left(\mathrm{CH}_{3}\right) ; 32.2\left(\mathrm{CH}_{2}\right) ; 32.1\left(\mathrm{CH}_{2}\right) ; 31.7\left(\mathrm{CH}_{2}\right) ; 28.4\left(\mathrm{CH}_{2}\right)$; $18.9\left(\mathrm{CH}_{2}\right) .-\mathrm{IR}$ (neat): $v\left(\mathrm{~cm}^{-1}\right)=3466.9(\mathrm{C}-\mathrm{OH}) ; 1741.5(\mathrm{C}=\mathrm{O}), 1166.3(\mathrm{C}-\mathrm{O}) .-\mathrm{MS}$ $\left(\mathrm{Cl}, \mathrm{NH}_{3}\right) \mathrm{m} / \mathrm{z}=\left[\mathrm{MNH}_{4}\right]^{+}=202 ;[\mathrm{MH}]^{+}=185 ;\left[\mathrm{MH}-\mathrm{H}_{2} \mathrm{O}\right]^{+}=167$.

[2-(1-Nitro-cyclohex-2-enyl)-ethanesulfonyl]-benzene (1j): A solution of 1-

nitrocyclohexene 6 (282 mg, $2.218 \mathrm{mmol}, 1 \mathrm{eq}$.), phenyl vinyl sulfone (746 mg, 4.436 $\mathrm{mmol}$, 2eq.) and $\mathrm{DBU}(0.03 \mathrm{ml}, 0.222 \mathrm{mmol}, 0.1$ eq.) in acetonitrile ( $3 \mathrm{ml})$ was stirred at room temp. overnight. The acetonitrile was evaporated under reduced pressure. The crude product was purified by flash chromatography (PE/EA 8:2 to 6:4) to give $619 \mathrm{mg}(95 \%)$ of $1 \mathrm{j}$ as a colourless oil. $-{ }^{1} \mathrm{H}$ NMR: $\delta=7.89(\mathrm{~d}, \mathrm{~J}=8 \mathrm{~Hz}, 2 \mathrm{H}, 2 \mathrm{CH}=)$; $7.70(\mathrm{t}, \mathrm{J}=8 \mathrm{~Hz}, 1 \mathrm{H}, \mathrm{CH}=) ; 7.60(\mathrm{t}, \mathrm{J}=8 \mathrm{~Hz}, 2 \mathrm{H}, 2 \mathrm{CH}=) ; 6.14(\mathrm{~m}, 1 \mathrm{H}, \mathrm{CH}=) ; 5.78(\mathrm{~m}$,

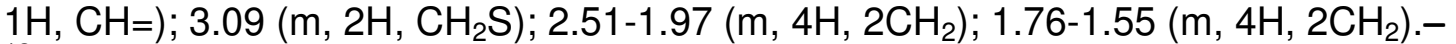
${ }^{13} \mathrm{C}$ NMR: $\delta=138.3$ (Cq.); $135.4(\mathrm{CH}=) ; 134.1(\mathrm{CH}=) ; 129.4(2 \mathrm{CH}=) ; 127.9(2 \mathrm{CH}=) ;$ $123.8(\mathrm{CH}=)$; 87.3 (Cq.); $51.01\left(\mathrm{CH}_{2}-\mathrm{S}\right) ; 32.8\left(\mathrm{CH}_{2}\right) ; 31.6\left(\mathrm{CH}_{2}\right) ; 24.5\left(\mathrm{CH}_{2}\right) ; 18.5$ $\left(\mathrm{CH}_{2}\right) .-\mathrm{IR}($ neat $): v\left(\mathrm{~cm}^{-1}\right)=1543\left(\mathrm{C}-\mathrm{NO}_{2}\right) ; 1327\left(\mathrm{C}-\mathrm{SO}_{2}\right) ; 1154\left(\mathrm{C}-\mathrm{SO}_{2}\right) .-\mathrm{MS}(\mathrm{Cl}$, $\left.\mathrm{NH}_{3}\right) \mathrm{m} / \mathrm{z}=\left[\mathrm{MH}+\mathrm{NH}_{3}\right]^{+}=313 ;[\mathrm{MH}]^{+}=296$. 
3-(2-Benzenesulfonyl-ethyl)-cyclohex-2-enol (3j): DABCO (57 mg, $0.508 \mathrm{mmol}$, 1.5 eq.) was added to a solution of $1 \mathbf{j}(100 \mathrm{mg}, 0.339 \mathrm{mmol}, 1$ eq.) in $1,2-$

dichlorobenzene $(1.6 \mathrm{ml})$. The resultant mixture was heated at reflux for $30 \mathrm{~min}$. The crude product was purified by flash chromatography (PE/EA 6:4 to 5:5) to give $58 \mathrm{mg}$ (64\%) of the alcohol $3 \mathbf{j}$ as a colourless oil. ${ }^{1} \mathrm{H}$ NMR: $\delta=7.93(\mathrm{~d}, \mathrm{~J}=8 \mathrm{~Hz}, 2 \mathrm{H}, 2 \mathrm{CH}=$ ); $7.69(\mathrm{t}, \mathrm{J}=8 \mathrm{~Hz}, 1 \mathrm{H}, \mathrm{CH}=) ; 7.60(\mathrm{t}, \mathrm{J}=8 \mathrm{~Hz}, 2 \mathrm{H}, 2 \mathrm{CH}=) ; 5.48(\mathrm{~s} \mathrm{br}, 1 \mathrm{H}, \mathrm{CH}=) ; 4.16(\mathrm{~s}$ br, $1 \mathrm{H}, \mathrm{CHO}) ; 3.23\left(\mathrm{~m}, 2 \mathrm{H}, \mathrm{CH}_{2} \mathrm{~S}\right) ; 2.41\left(\mathrm{~m}, 2 \mathrm{H}, \mathrm{CH}_{2}\right) ; 1.92-1.49\left(\mathrm{~m}, 6 \mathrm{H}, 3 \mathrm{CH}_{2}\right) .{ }^{13} \mathrm{C}$ NMR: $\delta=138.9$ (Cq.); 137.7 (Cq.); $133.8(\mathrm{CH}=) ; 129.5(2 \mathrm{CH}=) ; 128.0(2 \mathrm{CH}=) ; 125.9$ $(\mathrm{CH}=) ; 65.5(\mathrm{CH}-\mathrm{O}) ; 54.3\left(\mathrm{CH}_{2} \mathrm{~S}\right) ; 31.5\left(\mathrm{CH}_{2}\right) ; 29.9\left(\mathrm{CH}_{2}\right) ; 28.3\left(\mathrm{CH}_{2}\right) ; 18.9\left(\mathrm{CH}_{2}\right)$. $-\mathrm{IR}$ (neat): $v\left(\mathrm{~cm}^{-1}\right)=3527(\mathrm{C}-\mathrm{OH}) ; 1324\left(\mathrm{C}-\mathrm{SO}_{2}\right) ; 1153\left(\mathrm{C}^{-\mathrm{SO}_{2}}\right) .-\mathrm{MS}\left(\mathrm{Cl}, \mathrm{NH}_{3}\right) \mathrm{m} / \mathrm{z}=$ $\left[\mathrm{MH}+\mathrm{NH}_{3}\right]^{+}=284 ;[\mathrm{MH}]^{+}=267 ;\left[\mathrm{MH}-\mathrm{H}_{2} \mathrm{O}\right]^{+}=249$.

Trans-2(1,3-Isopropylidendiacetat)-5-phenyl-cyclohexanol (3k): DABCO (139 $\mathrm{mg}, 1.241 \mathrm{mmol}, 1.5 \mathrm{eq}$.) was added to a solution of $1 \mathbf{k}(299 \mathrm{mg}, 0.827 \mathrm{mmol}, 1 \mathrm{eq}$.) in 1,2-dichlorobenzene (2 ml). The resultant mixture was heated at reflux for $2 \mathrm{~h} 1 / 2$. The crude product was purified by flash chromatography (PE/EA 8:2 to 6:4) to give $178 \mathrm{mg}(65 \%)$ of the alcohol 3k as a colourless oil. ${ }^{1} \mathrm{H}$ NMR: $\delta=7.4-7.1(\mathrm{~m}, 5 \mathrm{H}$, $5 \mathrm{CH}=) ; 5.10\left(\mathrm{~d}, \mathrm{~J}_{\mathrm{AB}}=11.9 \mathrm{~Hz}, 1 \mathrm{H}, \mathrm{CH} 2 \mathrm{O}\right) ; 5.06$ (s br, $\left.1 \mathrm{~h}, \mathrm{CHO}\right) ; 4.71$ (d, $\mathrm{J}=2.5 \mathrm{~Hz}$, $\left.2 \mathrm{H}, \mathrm{CH}_{2} \mathrm{O}\right) ; 4.56\left(\mathrm{~d}, \mathrm{~J}_{\mathrm{AB}}=11.9,2 \mathrm{H}, \mathrm{CH}_{2} \mathrm{O}\right) ; 3.4-3.1(\mathrm{~m}, 1 \mathrm{H}, \mathrm{CH}) ; 2.8-2.5(\mathrm{~m}, 2 \mathrm{H}$, $\left.\mathrm{CH}_{2}\right)$; 2.3-1.9 (m, 2H, $\left.\mathrm{CH}_{2}\right)$; $2.05\left(\mathrm{~s}, 6 \mathrm{H}, 2 \mathrm{CH}_{3}\right) ; 1.8-1.5\left(\mathrm{~m}, 2 \mathrm{H}, \mathrm{CH}_{2}\right) .-{ }^{13} \mathrm{C} \mathrm{NMR:} \delta=$ 171.5 (CO); 171.1 (CO); 147.4 (Cq.); 145.9 (Cq.); $128.5(2 \mathrm{CH}=) ; 127.0(2 \mathrm{CH}=) ; 126.3$ $(\mathrm{CH}=) ; 122.9$ (Cq.); $65.8(\mathrm{CHO}) ; 61.9\left(\mathrm{CH}_{2} \mathrm{O}\right) ; 61.7\left(\mathrm{CH}_{2} \mathrm{O}\right) ; 41.0(\mathrm{CH}) ; 37.4\left(\mathrm{CH}_{2}\right)$; $34.4\left(\mathrm{CH}_{2}\right) ; 25.8\left(\mathrm{CH}_{2}\right) ; 21.2\left(\mathrm{CH}_{3}\right) ; 21.1\left(\mathrm{CH}_{3}\right)$. IR (neat): $v\left(\mathrm{~cm}^{-1}\right)=3495(\mathrm{C}-\mathrm{OH})$; $1737(\mathrm{C}=\mathrm{O})$; $1234(\mathrm{C}-\mathrm{OH})$.- Anal Calcd. for $\mathrm{C}_{18} \mathrm{H}_{24} \mathrm{O}_{5} \mathrm{C}$ (68.66), $\mathrm{H}$ (7.28); found $\mathrm{C}$ (68.27) $\mathrm{H}(7.19)$.

4-(1-Nitro-6-phenyl-cyclohex-2-enyl)-butan-2-one (11): A solution of 9 (113 mg, $0.557 \mathrm{mmol}, 1$ eq.), methyl vinyl ketone $(0.091 \mathrm{ml}, 1.113 \mathrm{mmol}, 2 \mathrm{eq}$.) and DBU $(0.008 \mathrm{ml}, 0.056 \mathrm{mmol}, 0.1$ eq. $)$ in acetonitrile $(0.84 \mathrm{ml})$ was stirred at room temp. for $3 \mathrm{~h}$. The acetonitrile was evaporated under reduced pressure. The crude product was purified by flash chromatography (PE/EA 8:2) to give $79 \mathrm{mg}(52 \%)$ of $1 \mathrm{I}$ as a colourless oil. $-{ }^{1} \mathrm{H}$ NMR: $\delta=7.27(\mathrm{~m}, 3 \mathrm{H}, 3 \mathrm{CH}=) ; 7.13(\mathrm{~m}, 2 \mathrm{H}, 2 \mathrm{CH}=) ; 6.26(\mathrm{dt}, \mathrm{J}=$ $10.0 \mathrm{~Hz}, \mathrm{~J}=3.8 \mathrm{~Hz}, 1 \mathrm{H}, \mathrm{CH}=) ; 5.93(\mathrm{~m}, 1 \mathrm{H}, \mathrm{CH}=) ; 3.19(\mathrm{dd}, \mathrm{J}=9.2 \mathrm{~Hz}, \mathrm{~J}=3.0 \mathrm{~Hz}, 1 \mathrm{H}$, $\mathrm{CH}) ; 2.59-1.93\left(\mathrm{~m}, 8 \mathrm{H}, 4 \mathrm{CH}_{2}\right) ; 2.16\left(\mathrm{~s}, 3 \mathrm{H}, \mathrm{CH}_{3}\right) .{ }^{13} \mathrm{C} \mathrm{NMR:} \delta=206.5(\mathrm{CO}) ; 139.2$ (Cq.); $134.7(\mathrm{CH}=) ; 128.5(2 \mathrm{CH}=) ; 127.9(2 \mathrm{CH}=) ; 127.6(\mathrm{CH}=) ; 124.01(\mathrm{CH}=) ; 91.4$ (Cq.); $48.6(\mathrm{CH}) ; 37.9\left(\mathrm{CH}_{2}\right) ; 32.3\left(\mathrm{CH}_{2}\right) ; 30.1\left(\mathrm{CH}_{3}\right) ; 24.9\left(\mathrm{CH}_{2}\right) ; 24.1\left(\mathrm{CH}_{2}\right)$. I IR (neat): $v\left(\mathrm{~cm}^{-1}\right)=1723(\mathrm{C}=\mathrm{O}) ; 1540\left(\mathrm{C}-\mathrm{NO}_{2}\right) .-\mathrm{MS}\left(\mathrm{Cl}, \mathrm{NH}_{3}\right) \mathrm{m} / \mathrm{z}=\left[\mathrm{MH}+\mathrm{NH}_{3}\right]^{+}=373$; $[\mathrm{MH}]^{+}=356 ;\left[\mathrm{MH}-\mathrm{NO}_{2}\right]^{+}=310$.

4-(3-Hydroxy-6-phenyl-cyclohex-1-enyl)-butan-2-one (3I): DABCO (69 mg, 0.615 $\mathrm{mmol}, 1.5$ eq.) was added to a solution of $1 \mathrm{l}(112 \mathrm{mg}, 0.410 \mathrm{mmol}, 1 \mathrm{eq}$.) in $1,2-$ dichlorobenzene $(1.6 \mathrm{ml})$. The resultant mixture was heated at reflux for $30 \mathrm{~min}$. The crude product was purified by flash chromatography (PE/EA 6:4 to 5:5) to give $84 \mathrm{mg}$ $(84 \%)$ of the alcohol $3 \mathrm{l}$ as a colourless oil. $-{ }^{1} \mathrm{H}$ NMR: $\delta=7.27(\mathrm{~m}, 5 \mathrm{H}, 5 \mathrm{CH}=) ; 5.77$ ( 
br, $1 \mathrm{H}, \mathrm{CH}=$ ); 4.34 (s br, $1 \mathrm{H}, \mathrm{CHO}) ; 3.35$ (s br, $1 \mathrm{H}, \mathrm{CH}) ; 2.53\left(\mathrm{~m}, 2 \mathrm{H}, \mathrm{CH}_{2}\right) ; 2.18-$ $1.59(\mathrm{~m}, 6 \mathrm{H}, 3 \mathrm{CH} 2) ; 2.12\left(\mathrm{~s}, 3 \mathrm{H}, \mathrm{CH}_{3}\right) .-{ }^{13} \mathrm{C} \mathrm{NMR:} \delta=208.1$ (CO); 143.6 (Cq.); 141.2 (Cq.); $128.4(4 \mathrm{CH}=) ; 127.1(\mathrm{CH}=) ; 126.4(\mathrm{CH}=) ; 66.6(\mathrm{CHO}) ; 44.5(\mathrm{CH}) ; 41.6\left(\mathrm{CH}_{2}\right)$; $29.8\left(\mathrm{CH}_{3}\right) ; 29.5\left(\mathrm{CH}_{2}\right) ; 29.3\left(\mathrm{CH}_{2}\right) ; 28.2\left(\mathrm{CH}_{2}\right)$ - IR (neat): $v\left(\mathrm{~cm}^{-1}\right)=3618(\mathrm{C}-\mathrm{OH})$; $1721(\mathrm{C}=\mathrm{O}) .-\left[\mathrm{MH}+\mathrm{NH}_{3}\right]^{+}=262 ;[\mathrm{MH}]^{+}=245 ;\left[\mathrm{MH}-\mathrm{H}_{2} \mathrm{O}\right]^{+}=227$.

(S)-6-Methoxymethoxy-1-nitro-cyclohexene (10): Dimethoxymethane (5.45 ml, $61.534 \mathrm{mmol}$, 22eq.) and $\mathrm{P}_{2} \mathrm{O}_{5}(2.774 \mathrm{~g}, 19.540 \mathrm{mmol}, 6.97 \mathrm{eq})$ were added to a solution of 8 (400 mg, $2.797 \mathrm{mmol}, 1$ eq.) in anhydrous $\mathrm{CHCl}_{3}(16.4 \mathrm{ml})$. The mixture was stirred at room temp. for $1 \mathrm{~h}$. The resulting solution was neutralised by addition of sat. aq. $\mathrm{NaHCO}_{3}$ at $0^{\circ} \mathrm{C}$. The solution was extracted with 1,2-dichloromethane, dried over anhydrous $\mathrm{Na}_{2} \mathrm{SO}_{4}$ and filtered. The solvent was removed in vacuo. The crude product was purified by flash chromatography $\left(\mathrm{CH}_{2} \mathrm{Cl}_{2} / \mathrm{EA} \mathrm{9:1)}\right.$ to give $326 \mathrm{mg}$ $(63 \%)$ of 10 as a colourless oil. $-{ }^{1} \mathrm{H}$ NMR: $\delta=7.44(\mathrm{dd}, \mathrm{J}=5.2 \mathrm{~Hz}, \mathrm{~J}=2.8 \mathrm{~Hz}, 1 \mathrm{H}$, $\mathrm{CH}=$ ); 4.82 (s br, $1 \mathrm{H}, \mathrm{CHO}) ; 4.83\left(\mathrm{~d}, \mathrm{~J}_{\mathrm{AB}}=6.8 \mathrm{~Hz}, 1 \mathrm{H}, \mathrm{CH}_{2}\right) ; 4.71\left(\mathrm{~d}, \mathrm{~J}_{\mathrm{AB}}=6.8 \mathrm{~Hz}, 1 \mathrm{H}\right.$, $\left.\mathrm{CH}_{2}\right) ; 3.96\left(\mathrm{~s}, 3 \mathrm{H}, \mathrm{CH}_{3}\right) ; 2.45\left(\mathrm{dtd}, \mathrm{J}=20.3 \mathrm{~Hz}, \mathrm{~J}=5.2 \mathrm{~Hz}, \mathrm{~J}=2.0 \mathrm{~Hz}, 1 \mathrm{H}, \mathrm{CH}_{2}\right) ; 2.23$ $\left(\mathrm{m}, 1 \mathrm{H}, \mathrm{CH}_{2}\right) ; 2.13\left(\mathrm{~m}, 1 \mathrm{H}, \mathrm{CH}_{2}\right) ; 2.13\left(\mathrm{~m}, 1 \mathrm{H}, \mathrm{CH}_{2}\right) ; 1.73\left(\mathrm{~m}, 2 \mathrm{H}, \mathrm{CH}_{2}\right) ; 1.58(\mathrm{tt}, \mathrm{J}=$ $\left.13.6 \mathrm{~Hz}, \mathrm{~J}=3.6 \mathrm{~Hz}, 1 \mathrm{H}, \mathrm{CH}_{2}\right) .-{ }^{13} \mathrm{C} \mathrm{NMR}: \delta=149.6(\mathrm{Cq}.) ; 138.1(\mathrm{CH}=) ; 96.6$ $\left(\mathrm{OCH}_{2} \mathrm{O}\right) ; 67.2(\mathrm{CHO}) ; 55.7\left(\mathrm{CH}_{3}\right) ; 28.6\left(\mathrm{CH}_{2}\right) ; 24.9\left(\mathrm{CH}_{2}\right) ; 15.7\left(\mathrm{CH}_{2}\right)$. - IR (neat): $v$ $\left(\mathrm{cm}^{-1}\right)=1527\left(\mathrm{C}-\mathrm{NO}_{2}\right), 1343(\mathrm{C}-\mathrm{O}) .-\mathrm{MS}\left(\mathrm{Cl}, \mathrm{NH}_{3}\right) \mathrm{m} / \mathrm{z}=\left[\mathrm{MH}+\mathrm{NH}_{3}\right]^{+}=205 ;[\mathrm{MH}]^{+}=$ 188.

[(Z)-(R)-3-((S)-1-Methoxymethoxy-ethyl)-3-nitro-hex-4-ene-1-sulfonyl]-benzene (1m): A solution of 1-nitrocyclohexene 10 (164 mg, $0.877 \mathrm{mmol}, 1$ eq.), phenyl vinyl sulfone $295 \mathrm{mg}, 1.754 \mathrm{mmol}$, 2eq.) and DBU (0.026 ml, $0.175 \mathrm{mmol}, 0.1 \mathrm{eq}$.) in acetonitrile $(1.3 \mathrm{ml})$ was stirred at room temp. overnight. The acetonitrile was evaporated under reduced pressure. The crude product was purified by flash chromatography (PE/EA 7:3) to give $193 \mathrm{mg} \mathrm{(62 \% )} \mathrm{of} 1 \mathrm{~m}$ as a colourless oil. $-{ }^{1} \mathrm{H}$ NMR: $\delta=7.91(\mathrm{~d}, 2 \mathrm{H}, \mathrm{J}=7.6 \mathrm{~Hz}, 2 \mathrm{CH}=) ; 7.71(\mathrm{t}, 1 \mathrm{H}, \mathrm{J}=7.6 \mathrm{HZ}, \mathrm{CH}=) ; 7.61(\mathrm{t}, 2 \mathrm{H}$, $\mathrm{J}=7.6 \mathrm{~Hz}, 2 \mathrm{CH}=) ; 6.13(\mathrm{dt}, 1 \mathrm{H}, \mathrm{J}=10.4 \mathrm{~Hz}, \mathrm{~J}=3.6 \mathrm{~Hz}, \mathrm{CH}=) ; 5.84(\mathrm{~d} \mathrm{br}, 1 \mathrm{H}, \mathrm{J}=$ $10.4 \mathrm{~Hz}, \mathrm{CH}=)$; $4.64\left(\mathrm{~d}, \mathrm{~J}_{\mathrm{AB}}=7.2 \mathrm{~Hz}, 1 \mathrm{H}, \mathrm{CH}_{2} \mathrm{O}\right) ; 4.55\left(\mathrm{~d}, \mathrm{~J}_{\mathrm{AB}}=7.2 \mathrm{~Hz}, 1 \mathrm{H}, \mathrm{CH}_{2} \mathrm{O}\right.$ ); 4.09 (d br, $1 \mathrm{H}, \mathrm{J}=5.2 \mathrm{~Hz}, \mathrm{CHO}) ; 3.29\left(\mathrm{~s}, 3 \mathrm{H}, \mathrm{CH}_{3} \mathrm{O}\right) ; 3.21(\mathrm{dt}, 1 \mathrm{H}, \mathrm{J}=13.2 \mathrm{~Hz}, \mathrm{~J}=$ $4.4 \mathrm{~Hz}, \mathrm{CH}_{2} \mathrm{~S}$ ); 2.97 (dt, $\left.1 \mathrm{H}, \mathrm{J}=13.2 \mathrm{~Hz}, \mathrm{~J}=4.0 \mathrm{~Hz}, \mathrm{CH}_{2} \mathrm{~S}\right) ; 2.61(\mathrm{dt}, 1 \mathrm{H}, \mathrm{J}=13.6$ $\left.\mathrm{Hz}, \mathrm{J}=4.0 \mathrm{~Hz}, \mathrm{CH}_{2}\right) ; 2.35\left(\mathrm{~m}, 2 \mathrm{H}, \mathrm{CH}_{2}\right) ; 2.14\left(\mathrm{~m}, 2 \mathrm{H}, \mathrm{CH}_{2}\right) ; 1.85\left(\mathrm{~m}, 1 \mathrm{H}, \mathrm{CH}_{2}\right) .-{ }^{13} \mathrm{C}$ NMR: $\delta=138.3$ (Cq.); $134.1(\mathrm{CH}=) ; 134.0(\mathrm{CH}=) ; 129.4(2 \mathrm{CH}=) ; 137.9(2 \mathrm{CH}=)$; $131.0(\mathrm{CH}=) ; 95.7$ (Cq.); $89.6\left(\mathrm{OCH}_{2} \mathrm{O}\right), 75.4(\mathrm{CHO}) ; 55.9\left(\mathrm{OCH}_{3}\right) ; 50.9\left(\mathrm{CH}_{2} \mathrm{~S}\right) ; 31.5$ $\left(\mathrm{CH}_{2}\right) ; 23.3\left(\mathrm{CH}_{2}\right) ; 21.4\left(\mathrm{CH}_{2}\right)$. - IR (neat): $v\left(\mathrm{~cm}^{-1}\right)=1551\left(\mathrm{C}-\mathrm{NO}_{2}\right) ; 1327(\mathrm{C}-\mathrm{O}) ; 1327$ $\left(\mathrm{C}-\mathrm{SO}_{2}\right) ; 1155.0\left(\mathrm{C}-\mathrm{SO}_{2}\right) .-\mathrm{MS}\left(\mathrm{Cl}, \mathrm{NH}_{3}\right) \mathrm{m} / \mathrm{z}=\left[\mathrm{MH}+\mathrm{NH}_{3}\right]^{+}=373 ;[\mathrm{MH}]^{+}=356 ;[\mathrm{MH}-$ $\left.\mathrm{NO}_{2}\right]^{+}=310$.

(1R,4S)-3-(2-Benzenesulfonyl-ethyl)-4-methoxymethoxy-cyclohex-2-enol (3m): DABCO (67 mg, $0.363 \mathrm{mmol}, 1.5$ eq.) was added to a solution of $1 \mathrm{~m} \mathrm{(86} \mathrm{mg,}$ $0.242 \mathrm{mmol}, 1$ eq.) in 1,2-dichlorobenzene $(1.6 \mathrm{ml})$. The resultant mixture was heated at reflux for $30 \mathrm{~min}$. The crude product was purified by flash chromatography (PE/EA $8: 2$ to $6: 4)$ to give $54 \mathrm{mg}(68 \%)$ of the alcohol $3 \mathrm{~m}$ as a colourless oil. $-{ }^{1} \mathrm{H}$ NMR: $\delta=$ 
$7.92(\mathrm{~d}, 2 \mathrm{H}, \mathrm{J}=7.2 \mathrm{~Hz}, 2 \mathrm{CH}=) ; 7.67(\mathrm{t}, 1 \mathrm{H}, \mathrm{J}=7.6 \mathrm{~Hz}, \mathrm{CH}=) ; 7.58(\mathrm{t}, 2 \mathrm{H}, \mathrm{J}=7.6 \mathrm{~Hz}$, $2 \mathrm{CH}=) ; 5.61(\mathrm{~s}, 1 \mathrm{H}, \mathrm{CH}=) ; 4.69\left(\mathrm{~d}, \mathrm{~J}_{\mathrm{AB}}=7.2 \mathrm{~Hz}, 1 \mathrm{H}, \mathrm{CH}_{2} \mathrm{O}\right) ; 4.54\left(\mathrm{~d}, \mathrm{~J}_{\mathrm{AB}}=7.2 \mathrm{~Hz}\right.$, $1 \mathrm{H}, \mathrm{CH}_{2} \mathrm{O}$ ); 4.08 (s br, $\left.1 \mathrm{H}, \mathrm{CHO}\right) ; 3.84(\mathrm{~s}, 1 \mathrm{H}, \mathrm{CHO}) ; 3.33\left(\mathrm{~s}, 3 \mathrm{H}, \mathrm{CH}_{3} \mathrm{O}\right) ; 3.28(\mathrm{~m}$, $\left.2 \mathrm{H}, \mathrm{CH}_{2} \mathrm{~S}\right) ; 2.55\left(\mathrm{~m}, 2 \mathrm{H}, \mathrm{CH}_{2}\right) ; 1.84\left(\mathrm{~m}, 2 \mathrm{H}, \mathrm{CH}_{2}\right) ; 1.63-1.41\left(\mathrm{~m}, 4 \mathrm{H}, 2 \mathrm{CH}_{2}\right) .-{ }^{13} \mathrm{C}$ NMR: $\delta=139.1$ (Cq.); 137.0 (Cq.); $133.7(\mathrm{CH}=) ; 130.9(\mathrm{CH}=) ; 129.3(2 \mathrm{CH}=) ; 128.1$ $(2 \mathrm{CH}=) ; 95.6\left(\mathrm{CH}_{2}\right) ; 71.9(\mathrm{CHO}) ; 66.3(\mathrm{CHO}) ; 55.9\left(\mathrm{OCH}_{3}\right) ; 54.8\left(\mathrm{CH}_{2}\right) ; 27.7\left(\mathrm{CH}_{2}\right)$; $26.9\left(\mathrm{CH}_{2}\right) ; 25.3\left(\mathrm{CH}_{2}\right)$ - - IR (neat): $v\left(\mathrm{~cm}^{-1}\right)=3460(\mathrm{C}-\mathrm{OH}) ; 1323(\mathrm{C}-\mathrm{O}) ; 1323(\mathrm{C}-$ $\left.\mathrm{SO}_{2}\right) ; 1152\left(\mathrm{C}-\mathrm{SO}_{2}\right) .-\mathrm{MS}\left(\mathrm{Cl}, \mathrm{NH}_{3}\right) \mathrm{m} / \mathrm{z}=\left[\mathrm{MH}+\mathrm{NH}_{3}\right]^{+}=344 ;[\mathrm{MH}]^{+}=327 ;\left[\mathrm{MH}-\mathrm{H}_{2} \mathrm{O}\right]^{+}$ $=309 ;[\mathrm{MH}-\mathrm{OMOM}]^{+}=266$.

Dithiocarbonic acid [2,2-diethoxy-1-(2-oxo-cyclohexylmethyl)-ethyl] ester ethyl ester (12) : Lauroyl peroxide (DLP) $(91 \mathrm{mg}, 0.229 \mathrm{mmol}, 5 \%)$, was added to a refluxing degassed solution of xanthate 11 (1 g, $4.587 \mathrm{mmol}, 1 \mathrm{eq}$.), 3,3-diethoxypropene (1.05 ml, $6.881 \mathrm{mmol}, 1.5 \mathrm{eq}$.) in 1,2-dichloroethane (9.2 ml). Additional DLP (91 $\mathrm{mg}, 0.229 \mathrm{mmol}, 5 \%$ ) was added every $1 \mathrm{~h}$ to the refluxing solution until no starting material was observed by TLC. After cooling to room temp., the solvent was removed in vacuo. The crude product was purified by flash chromatography (PE/EA 8:2) to give $978 \mathrm{mg}$ of $12(61 \%)$ as a colourless oil and as a mixture of 2 diastereomers in a 1:1 ratio. $-{ }^{1} \mathrm{H}$ NMR: $\delta=4.57\left(\mathrm{~m}, 2 \mathrm{H}, \mathrm{CH}_{2} \mathrm{O}\right) ; 4.49$ and 4,47 (d, J $=2.8 \mathrm{~Hz}, 1 \mathrm{H}, \mathrm{CH}) ; 4.13$ and $4.01(\mathrm{dt}, \mathrm{J}=10.8 \mathrm{~Hz}, \mathrm{~J}=2.9 \mathrm{~Hz}, 1 \mathrm{H}, \mathrm{CHS}) ; 3.64(\mathrm{~m}, 2 \mathrm{H}$, $\left.\mathrm{CH}_{2} \mathrm{O}\right) ; 3.45\left(\mathrm{~m}, 2 \mathrm{H}, \mathrm{CH}_{2} \mathrm{O}\right) ; 2.52(\mathrm{~m}, 1 \mathrm{H}, \mathrm{CH}) ; 2.34-1.55\left(\mathrm{M}, 10 \mathrm{H}, 5 \mathrm{CH}_{2}\right) ; 1.41$ and $1.38\left(\mathrm{t}, \mathrm{J}=7.0 \mathrm{~Hz}, 3 \mathrm{H}, \mathrm{CH}_{3}\right) ; 1.22-1.10\left(\mathrm{~m}, 6 \mathrm{H}, 2 \mathrm{CH}_{3}\right) .-{ }^{13} \mathrm{C} \mathrm{NMR}: \delta=215.1$ and 214.8 (CS) ; 212.5 and $212.1(\mathrm{CO}) ; 104.1$ and $103.7(\mathrm{CH}) ; 69.9$ and $69.8\left(\mathrm{CH}_{2} \mathrm{O}\right)$; 64.4 and $63.9\left(\mathrm{CH}_{2} \mathrm{O}\right) ; 63.5$ and $63.2\left(\mathrm{CH}_{2} \mathrm{O}\right) ; 51.8$ and $51.1(\mathrm{CHS}) ; 47.6$ and 47.4 $(\mathrm{CH}) ; 42.1$ and $41.8\left(\mathrm{CH}_{2}\right) ; 35,3$ and $33.7\left(\mathrm{CH}_{2}\right) ; 28.3$ and $27.7\left(\mathrm{CH}_{2}\right) ; 28.2$ and $27.2\left(\mathrm{CH}_{2}\right) ; 25.1$ and $24.7\left(\mathrm{CH}_{2}\right) ; 15.0$ and $14.9\left(\mathrm{CH}_{3}\right) ; 14.9$ and $14.8\left(\mathrm{CH}_{3}\right) ; 13.6$ and $13.5\left(\mathrm{CH}_{3}\right)$.- IR (neat): $v\left(\mathrm{~cm}^{-1}\right)=1713(\mathrm{C}=\mathrm{O}) ; 1217(\mathrm{C}-\mathrm{S}) ; 1055(\mathrm{C}=\mathrm{S}) .-\mathrm{MS}(\mathrm{Cl}$, $\left.\mathrm{NH}_{3}\right) \mathrm{m} / \mathrm{z}=\left[\mathrm{MH}+\mathrm{NH}_{3}\right]^{+}=366 ;[\mathrm{MH}]^{+}=349 ;[\mathrm{MH}-\mathrm{OEt}]^{+}=304$.

2-(3,3-Diethoxy-propyl)-cyclohexanone (13) : A solution of 12 (978 mg, 2.810 $\mathrm{mmol}, 1$ eq.) in toluene $(28.1 \mathrm{ml})$ was heated at reflux for $30 \mathrm{~min}$ under nitrogen. The reaction mixture was allowed to cool to $100^{\circ} \mathrm{C}$ and tributylstannane $(1.13 \mathrm{ml}, 4.216$ mmol, 1.5 eq.) and AIBN (46 mg, $0.281 \mathrm{mmol}, 0.1$ eq.) were added. The reaction mixture was then stirred at $100^{\circ} \mathrm{C}$ for $1 \mathrm{~h}$. The solvent was removed in vacuo and the crude product was purified by flash chromatography (Pentane/EA 9.5:0.5 to 8:2) to ) to give $450 \mathrm{mg}$ of $13(70 \%)$ as a colourless oil. $-{ }^{1} \mathrm{H}$ NMR: $\delta=4.46(\mathrm{t}, \mathrm{J}=5.6 \mathrm{~Hz}, 1 \mathrm{H}$, $\mathrm{CH}) ; 3.63\left(\mathrm{~m}, 2 \mathrm{H}, \mathrm{CH}_{2} \mathrm{O}\right) ; 3.47\left(\mathrm{~m}, 2 \mathrm{H}, \mathrm{CH}_{2} \mathrm{O}\right) ; 2.40-2.24\left(\mathrm{~m}, 3 \mathrm{H}, \mathrm{CH} / \mathrm{CH}_{2}\right) ; 2.12$ $2.02\left(\mathrm{~m}, 2 \mathrm{H}, \mathrm{CH}_{2}\right) ; 1.81\left(\mathrm{~m}, 2 \mathrm{H}, \mathrm{CH}_{2}\right) ; 1.67\left(\mathrm{~m}, 4 \mathrm{H}, 2 \mathrm{CH}_{2}\right) ; 1.42$ - $1.22\left(\mathrm{~m}, 2 \mathrm{H}, \mathrm{CH}_{2}\right)$; $1.18\left(\mathrm{t}, \mathrm{J}=7.0 \mathrm{~Hz}, 6 \mathrm{H}, 2 \mathrm{CH}_{3}\right) \cdot-{ }^{13} \mathrm{C}$ NMR: $\delta=213.2(\mathrm{CO}) ; 102.9(\mathrm{CH}) ; 61.0\left(\mathrm{CH}_{2} \mathrm{O}\right)$; $60.8\left(\mathrm{CH}_{2} \mathrm{O}\right) ; 50.4(\mathrm{CH}) ; 42.0\left(\mathrm{CH}_{2}\right) ; 33.9\left(\mathrm{CH}_{2}\right) ; 31.2\left(\mathrm{CH}_{2}\right) ; 28.0\left(\mathrm{CH}_{2}\right) ; 24.9\left(\mathrm{CH}_{2}\right)$; $24.6\left(\mathrm{CH}_{2}\right) ; 15.3\left(\mathrm{CH}_{3}\right)$ - - IR (neat): $v\left(\mathrm{~cm}^{-1}\right)=1712(\mathrm{C}=\mathrm{O}) ; 1124(\mathrm{C}-\mathrm{O}) .-\mathrm{MS}\left(\mathrm{Cl}, \mathrm{NH}_{3}\right)$ $\mathrm{m} / \mathrm{z}=\left[\mathrm{MH}+\mathrm{NH}_{3}\right]^{+}=366 ;[\mathrm{MH}]^{+}=349 ;[\mathrm{MH}-\mathrm{OEt}]^{+}=184$.

6-(3,3-Diethoxy-propyl)-5-nitromethyl-cyclohexa-1,2,4-triene (14) : N,Ndimethylethylenediamine (2 drops) was added to a solution of 13 (400 mg, 1.754 
$\mathrm{mmol}, 1$ eq.) in nitromethane $(8.8 \mathrm{ml})$. The solution was heated at reflux for $12 \mathrm{~h}$. The mixture was concentrated and the crude product was purified by flash chromatography (PE/EA 8:2) to give $310 \mathrm{mg}$ of 14 (65\%) as a transparent oil and as a mixture of 2 compounds.

(a) $-{ }^{1} \mathrm{H}$ NMR: $\delta=5.95$ (t, J = $\left.3.4 \mathrm{~Hz}, 1 \mathrm{H}, \mathrm{CH}=\right) ; 5.03\left(\mathrm{~d}, \mathrm{~J}_{\mathrm{AB}}=12.8 \mathrm{~Hz}, 1 \mathrm{H}, \mathrm{CH}_{2} \mathrm{~N}\right)$; $4.72\left(\mathrm{~d}, \mathrm{~J}_{\mathrm{AB}}=12.8 \mathrm{~Hz}, 1 \mathrm{H}, \mathrm{CH}_{2} \mathrm{~N}\right) ; 4.45(\mathrm{~m}, 1 \mathrm{H}, \mathrm{CH}) ; 3.63\left(\mathrm{~m}, 2 \mathrm{H}, \mathrm{CH}_{2} \mathrm{O}\right) ; 3.49(\mathrm{~m}$, $\left.2 \mathrm{H}, \mathrm{CH}_{2} \mathrm{O}\right) ; 2.20(\mathrm{~m}, 1 \mathrm{H}, \mathrm{CH}) ; 2.08\left(\mathrm{~m}, 2 \mathrm{H}, \mathrm{CH}_{2}\right) ; 1.72-1.32\left(\mathrm{~m}, 8 \mathrm{H}, 4 \mathrm{CH}_{2}\right) ; 1.20$ (t, J $\left.=7.2 \mathrm{~Hz}, 3 \mathrm{H}, \mathrm{CH}_{3}\right) .-{ }^{13} \mathrm{C}$ NMR: $\delta=134.8(\mathrm{CH}=) ; 131.9(\mathrm{Cq}.) ; 102.8(\mathrm{CH}) ; 80.6$ $\left(\mathrm{CH}_{2} \mathrm{~N}\right) ; 61.3\left(\mathrm{CH}_{2} \mathrm{O}\right) ; 61.2\left(\mathrm{CH}_{2} \mathrm{O}\right) ; 34.6(\mathrm{CH}) ; 30.9\left(\mathrm{CH}_{2}\right) ; 26.9\left(\mathrm{CH}_{2}\right) ; 26.5\left(\mathrm{CH}_{2}\right)$; $25.7\left(\mathrm{CH}_{2}\right) ; 18.6\left(\mathrm{CH}_{2}\right) ; 15.3\left(\mathrm{CH}_{3}\right)$. - IR (neat): $v\left(\mathrm{~cm}^{-1}\right)=1555\left(\mathrm{C}-\mathrm{NO}_{2}\right) ; 1371(\mathrm{C}-$ O). $-\mathrm{MS}\left(\mathrm{Cl}, \mathrm{NH}_{3}\right) \mathrm{m} / \mathrm{z}=\left[\mathrm{MH}+\mathrm{NH}_{3}\right]^{+}=289 ;[\mathrm{MH}]^{+}=272 ;\left[\mathrm{MH}-\mathrm{NO}_{2}\right]^{+}=226$.

(b) - ${ }^{1} \mathrm{H}$ NMR: $\delta=4.99\left(\mathrm{~s}, 2 \mathrm{H}, \mathrm{CH}_{2} \mathrm{~N}\right) ; 4.42(\mathrm{~m}, 1 \mathrm{H}, \mathrm{OCHO}) ; 3.62\left(\mathrm{~m}, 2 \mathrm{H}, \mathrm{CH}_{2} \mathrm{O}\right)$; $3.48\left(\mathrm{~m}, 2 \mathrm{H}, \mathrm{CH}_{2} \mathrm{O}\right) ; 2.17(\mathrm{~m}, 1 \mathrm{H}, \mathrm{CH}) ; 2.08\left(\mathrm{~m}, 2 \mathrm{H}, \mathrm{CH}_{2}\right) ; 1.72-1.32\left(\mathrm{~m}, 8 \mathrm{H}, 4 \mathrm{CH}_{2}\right)$; 1.19 (t, J = 7.2 Hz, 3H, $\mathrm{CH}_{3}$ ).- ${ }^{13} \mathrm{C}$ NMR: $\delta=134.8$ (Cq.); 121.5 (Cq.); 102.1 (CH); $77.7\left(\mathrm{CH}_{2} \mathrm{~N}\right) ; 61.3\left(\mathrm{CH}_{2} \mathrm{O}\right) ; 61.12\left(\mathrm{CH}_{2} \mathrm{O}\right) ; 34.6(\mathrm{CH}) ; 31.8\left(\mathrm{CH}_{2}\right) ; 29.6\left(\mathrm{CH}_{2}\right) ; 28.6$ $\left(\mathrm{CH}_{2}\right) ; 28.4\left(\mathrm{CH}_{2}\right) ; 22.6\left(\mathrm{CH}_{2}\right) ; 22.6\left(\mathrm{CH}_{2}\right) ; 15.3\left(\mathrm{CH}_{3}\right)$. - IR (neat): $v\left(\mathrm{~cm}^{-1}\right)=1555(\mathrm{C}-$ $\left.\mathrm{NO}_{2}\right) ; 1371(\mathrm{C}-\mathrm{O}) .-\mathrm{MS}\left(\mathrm{Cl}, \mathrm{NH}_{3}\right) \mathrm{m} / \mathrm{z}=\left[\mathrm{MH}+\mathrm{NH}_{3}\right]^{+}=289 ;[\mathrm{MH}]^{+}=272 ;\left[\mathrm{MH}-\mathrm{NO}_{2}\right]^{+}=$ 226.

\section{4-[6-(3,3-Diethoxy-propyl)-cyclohexa-1,3,4-trienyl]-4-nitro-butyric acid methyl ester}

(15): A solution of 14 (904 mg, $3.324 \mathrm{mmol}, 1$ eq.), acrylic acid methyl ester $(0.9 \mathrm{ml}$, $9.972 \mathrm{mmol}$, 3eq.) and $\mathrm{NEt}_{3}(0.14 \mathrm{ml}, 0.997 \mathrm{mmol}, 0.3$ eq.) in acetonitrile $(5 \mathrm{ml})$ was heated at reflux for $8 \mathrm{~h}$. The acetonitrile was evaporated under reduced pressure. The crude product was purified by flash chromatography (PE/EA 9:1) to give $750 \mathrm{mg}$ $(59 \%(82 \%))$ of 15 as a colourless oil and as a mixture of 2 diastereomers in a 6:4 radio. $-{ }^{1} \mathrm{H}$ NMR: $\delta=6.07$ and $5.94(\mathrm{t} \mathrm{br}, \mathrm{J}=3.6 \mathrm{~Hz}, 1 \mathrm{H}, \mathrm{CH}=) ; 9.95(\mathrm{~m}, 1 \mathrm{H}, \mathrm{CHN})$; $4.49(\mathrm{~m}, 1 \mathrm{H}, \mathrm{OCHO}) ; 3.70\left(\mathrm{~s} \mathrm{br}, 3 \mathrm{H}, \mathrm{OCH}_{3}\right) ; 3.65\left(\mathrm{~m}, 2 \mathrm{H}, \mathrm{CH}_{2} \mathrm{O}\right) ; 3.51(\mathrm{~m}, 2 \mathrm{H}$, $\left.\mathrm{CH}_{2} \mathrm{O}\right) ; 2.55$ - $1.98\left(\mathrm{~m}, 7 \mathrm{H}, \mathrm{CH} / 3 \mathrm{CH}_{2}\right) ; 1.75-1.29\left(\mathrm{~m}, 8 \mathrm{H}, 4 \mathrm{CH}_{2}\right) ; 1.22\left(\mathrm{~m}, 6 \mathrm{H}, 2 \mathrm{CH}_{3}\right)$. $-{ }^{13} \mathrm{C}$ NMR: $\delta=172.4$ and $172.3(\mathrm{CO}) ; 136.4$ and 135.3 (Cq.); 129.9 and 127.5 $(\mathrm{CH}=) ; 102.8(\mathrm{CH}) ; 89.2$ and $88.6(\mathrm{CHN}) ; 61.4$ and $61.1\left(\mathrm{CH}_{2} \mathrm{O}\right) ; 61.1$ and 60.9 $\left(\mathrm{CH}_{2} \mathrm{O}\right) ; 51.8\left(\mathrm{OCH}_{3}\right) ; 36.9$ and $35.5(\mathrm{CH}) ; 31.5$ and $31.3\left(\mathrm{CH}_{2}\right) ; 30.3$ and $30.3\left(\mathrm{CH}_{2}\right)$; 29.1 and $27.2\left(\mathrm{CH}_{2}\right) ; 26.9$ and $26.6\left(\mathrm{CH}_{2}\right) ; 26.0$ and $25.9\left(\mathrm{CH}_{2}\right) ; 25.4$ and $25.1\left(\mathrm{CH}_{2}\right)$; 17.6 and $17.4\left(\mathrm{CH}_{2}\right) ; 15.3\left(2 \mathrm{CH}_{3}\right) .-\mathrm{IR}$ (neat): $v\left(\mathrm{~cm}^{-1}\right)=1741(\mathrm{C}=\mathrm{O}) ; 1551\left(\mathrm{C}-\mathrm{NO}_{2}\right)$; $1372(\mathrm{C}-\mathrm{O}) .-\mathrm{MS}\left(\mathrm{Cl}, \mathrm{NH}_{3}\right) \mathrm{m} / \mathrm{z}=\left[\mathrm{MH}+\mathrm{NH}_{3}\right]^{+}=375 ;[\mathrm{MH}]^{+}=358 ;\left[\mathrm{MH}-\mathrm{NO}_{2}\right]^{+}=312$; $[\mathrm{MH}-2 \mathrm{OEt}]^{+}=268,[\mathrm{MH}-\mathrm{OMe}]^{+}=327$.

\section{4-Nitro-4-[6-(3-oxo-propyl)-cyclohexa-1,3,4-trienyl]-butyric acid methyl ester} (16): PTSA (115 mg $0.605 \mathrm{mmol}, 0.36$ eq.) was added to a solution of 15 (600 $\mathrm{mg}$ $, 1.681 \mathrm{mmol}, 1 \mathrm{eq}$.$) in acetone/water (2: 1,35 \mathrm{ml})$ and the resultant mixture was stirred for $2 \mathrm{~h}$ at room temp. The resulting solution was neutralised by addition of sat. aq. $\mathrm{Na}_{2} \mathrm{CO}_{3}$ and extracted with AcOEt. The mixture was then concentrated under reduced pressure and the crude product was purified by flash chromatography (PE/EA $7: 3)$ to give $280 \mathrm{mg}(59 \%)$ of 16 as a transparent oil and as a mixture of 2 diastereomers in a 6:4 radio. $-{ }^{1} \mathrm{H}$ NMR: $\delta=9.81(\mathrm{~s} b r, 1 \mathrm{H}, \mathrm{CH}=0) ; 6.11$ and $5.98(\mathrm{t}$, 
$\mathrm{J}=3.6 \mathrm{~Hz}, 1 \mathrm{H}, \mathrm{CH}=) ; 5.03(\mathrm{~m}, 1 \mathrm{H}, \mathrm{CHN}) ; 3.70\left(\mathrm{~s} \mathrm{br}, 3 \mathrm{H}, \mathrm{OCH}_{3}\right) ; 2.58-1.80(\mathrm{~m}, 9 \mathrm{H}$, $\left.\mathrm{CH} / 4 \mathrm{CH}_{2}\right) ; 1.56\left(\mathrm{~m}, 6 \mathrm{H}, 3 \mathrm{CH}_{2}\right) .-{ }^{13} \mathrm{C}$ NMR: $\delta=201.9$ and $201.7(\mathrm{CO}) ; 172.5$ and $172.4(\mathrm{CO}) ; 136.1$ and 134.9 (Cq.); 130.5 and $127.7(\mathrm{CH}=)$; 88.7 and $88.5(\mathrm{CHN})$; $51.9\left(\mathrm{OCH}_{3}\right) ; 41.8$ and $41.6\left(\mathrm{CH}_{2}\right) ; 36.4$ and $35.4(\mathrm{CH}) ; 30.3$ and $30.3\left(\mathrm{CH}_{2}\right) ; 29.7$ and $26.6\left(\mathrm{CH}_{2}\right) ; 25.8$ and $25.8\left(\mathrm{CH}_{2}\right) ; 25.4$ and $25.0\left(\mathrm{CH}_{2}\right) ; 24.2$ and $23.9\left(\mathrm{CH}_{2}\right) ; 17.4$ and $17.3\left(\mathrm{CH}_{2}\right)$. - IR (neat): $v\left(\mathrm{~cm}^{-1}\right)=1749(\mathrm{C}=\mathrm{O}) ; 1444\left(\mathrm{C}-\mathrm{NO}_{2}\right) ; 1252(\mathrm{C}-\mathrm{O}) .-\mathrm{MS}$ $\left(\mathrm{Cl}, \mathrm{NH}_{3}\right) \mathrm{m} / \mathrm{z}=\left[\mathrm{MH}+\mathrm{NH}_{3}\right]^{+}=301 ;[\mathrm{MH}]^{+}=284 ;\left[\mathrm{MH}-\mathrm{NO}_{2}\right]^{+}=238$.

\section{3-((1S,2S,4aS)-2-Acetoxy-1-nitro-1,2,3,4,4a,5,6,7-octahydro-naphthalen-1-yl)-} propionic acid methyl ester (1n): DBU $(0.03 \mathrm{ml}, 0.177 \mathrm{mmol}, 0.2 \mathrm{eq}$.) was added to a solution of 16 (250 mg, $0.883 \mathrm{mmol}, 1 \mathrm{eq}$.) in $1.3 \mathrm{ml}$ of acetonitrile and the resulting solution was stirred at room temp. for $30 \mathrm{~min}$. and then concentrated under reduced pressure.

$\mathrm{Ac}_{2} \mathrm{O}(0.4 \mathrm{ml}, 4.415 \mathrm{mmol}, 5$ eq.) and DMAP (11 mg, $0.088 \mathrm{mmol}, 10 \%)$ were added at $0^{\circ} \mathrm{C}$ to a solution of the alcohol in 1,2-dichloromethane $(2 \mathrm{ml})$ and the reaction mixture was stirred for 2 hours. $\mathrm{MeOH}$ was then added and the reaction mixture was quenched with sat. aq. $\mathrm{NaHCO}_{3}$. The organic layer was washed with brine, dried with anhydrous $\mathrm{Na}_{2} \mathrm{SO}_{4}$, filtered, and concentrated to give a crude oil which was purified using column chromatography (PE/EA $8: 2)$ to give $126 \mathrm{mg}(60 \%, 2$ steps) of $1 \mathrm{n}$ as a transparent oil. $-{ }^{1} \mathrm{H}$ NMR: $\delta=5.63(\mathrm{dd}, \mathrm{J}=11.6 \mathrm{~Hz}, \mathrm{~J}=4.8 \mathrm{~Hz}, 1 \mathrm{H}, \mathrm{CHO}) ; 5.33$ $(\mathrm{m}, 1 \mathrm{H}, \mathrm{CH}=) ; 3.71\left(\mathrm{~s}, 3 \mathrm{H}, \mathrm{OCH}_{3}\right) ; 2.79\left(\mathrm{~m}, 1 \mathrm{H}, \mathrm{CH}_{2}\right) ; 2.63\left(\mathrm{~m}, 1 \mathrm{H}, \mathrm{CH}_{2}\right) ; 2.40-2.01$ $\left(\mathrm{m}, 5 \mathrm{H}, \mathrm{CH} / 2 \mathrm{CH}_{2}\right) ; 1.99\left(\mathrm{~s}, 3 \mathrm{H}, \mathrm{CH}_{3}\right) ; 1.99-1.24\left(\mathrm{~m}, 8 \mathrm{H}, \mathrm{CH} / 4 \mathrm{CH}_{2}\right) .-{ }^{13} \mathrm{C} \mathrm{NMR}: \delta=$ 173.7 (CO); 169.3 (CO); 134.3 (Cq.); 125.3 (CH=); 98.6 (Cq.); 76.3 (CHO); 51.8 $\left(\mathrm{OCH}_{3}\right) ; 34.4(\mathrm{CH}) ; 30.4\left(\mathrm{CH}_{2}\right) ; 30.2\left(\mathrm{CH}_{2}\right) ; 28.9\left(\mathrm{CH}_{2}\right) ; 27.4\left(\mathrm{CH}_{2}\right) ; 25.3\left(\mathrm{CH}_{2}\right) ; 24.8$ $\left(\mathrm{CH}_{2}\right) ; 20.9\left(\mathrm{CH}_{3}\right) ; 20.3\left(\mathrm{CH}_{2}\right) .-\mathrm{IR}$ (neat): $v\left(\mathrm{~cm}^{-1}\right)=1750(\mathrm{C}=\mathrm{O}) ; 1547\left(\mathrm{C}-\mathrm{NO}_{2}\right) ; 1226$ (C-O).- MS $\left(\mathrm{Cl}, \mathrm{NH}_{3}\right) \mathrm{m} / \mathrm{z}=\left[\mathrm{MH}+\mathrm{NH}_{3}\right]^{+}=343 ;[\mathrm{MH}]^{+}=326 ;\left[\mathrm{MH}-\mathrm{NO}_{2}\right]^{+}=280$.

\section{3-((2S,8S, 4aS)-2-Acetoxy-8-hydroxy-2,3,4,4a,5,6,7,8-octahydro-naphthalen-1-} yl)-propionic acid methyl ester (3n): DABCO (62 mg, $0.554 \mathrm{mmol}, 1.5$ eq.) was added to a solution of $1 \mathrm{n}$ (120 mg, $0.369 \mathrm{mmol}, 1$ eq.) in 1,2-dichlorobenzene (1.6 $\mathrm{ml})$. The resultant mixture was heated at reflux for $30 \mathrm{~min}$. The crude product was purified by flash chromatography (PE/EA 6:4 to 5:5) to give $61 \mathrm{mg}(56 \%)$ of the

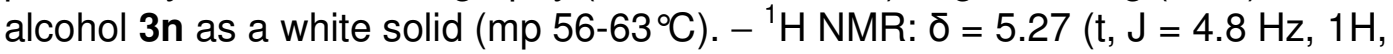
$\mathrm{CHO}) ; 4.38(\mathrm{~m}, 1 \mathrm{H}, \mathrm{CHO}) ; 3.68\left(\mathrm{~s}, 3 \mathrm{H}, \mathrm{CH}_{3}\right) ; 3.01-2.45\left(\mathrm{~m}, 4 \mathrm{H}, 2 \mathrm{CH}_{2}\right) ; 2.06(\mathrm{~s}, 3 \mathrm{H}$, $\left.\mathrm{CH}_{3}\right)$; 2.07-1.24 (m, 11H, CH/5CH $\mathrm{CH}_{2}-{ }^{13} \mathrm{C} \mathrm{NMR:} \delta=175.1$ (CO); 170.9 (CO); 144.7 (Cq.) 125.9 (Cq.); 72.1 (CHO); $70.8(\mathrm{CHO}) ; 51.8\left(\mathrm{OCH}_{3}\right) ; 36.4(\mathrm{CH}) ; 33.4\left(\mathrm{CH}_{2}\right)$; $32.1\left(\mathrm{CH}_{2}\right) ; 31.1\left(\mathrm{CH}_{2}\right) ; 26.5\left(\mathrm{CH}_{2}\right) ; 25.6\left(\mathrm{CH}_{2}\right) ; 23.5\left(\mathrm{CH}_{2}\right) ; 21.4\left(\mathrm{CH}_{2}\right) ; 21.3\left(\mathrm{CH}_{3}\right)$.IR (neat): $v\left(\mathrm{~cm}^{-1}\right)=3477(\mathrm{C}-\mathrm{OH}) ; 1734(\mathrm{C}=\mathrm{O}) ; 1239(\mathrm{C}-\mathrm{O}) .-\mathrm{MS}\left(\mathrm{Cl}, \mathrm{NH}_{3}\right) \mathrm{m} / \mathrm{z}=$ $\left[\mathrm{MH}+\mathrm{NH}_{3}\right]^{+}=314 ;[\mathrm{MH}]^{+}=297 ;\left[\mathrm{MH}-\mathrm{H}_{2} \mathrm{O}\right]^{+}=279 ;\left[\mathrm{MH}-\mathrm{Ac}_{2} \mathrm{O}\right]^{+}=238$. 


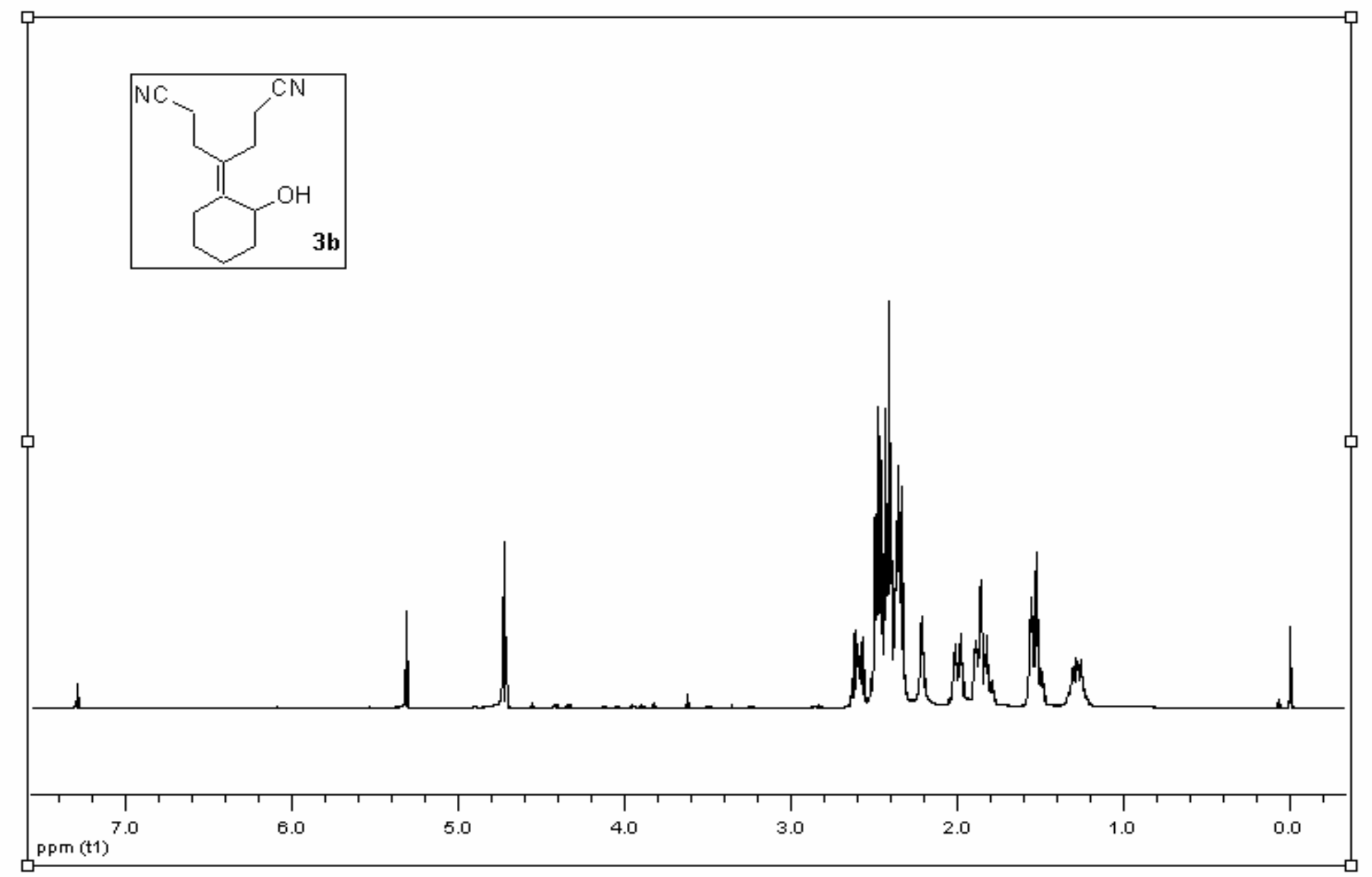

SI11 


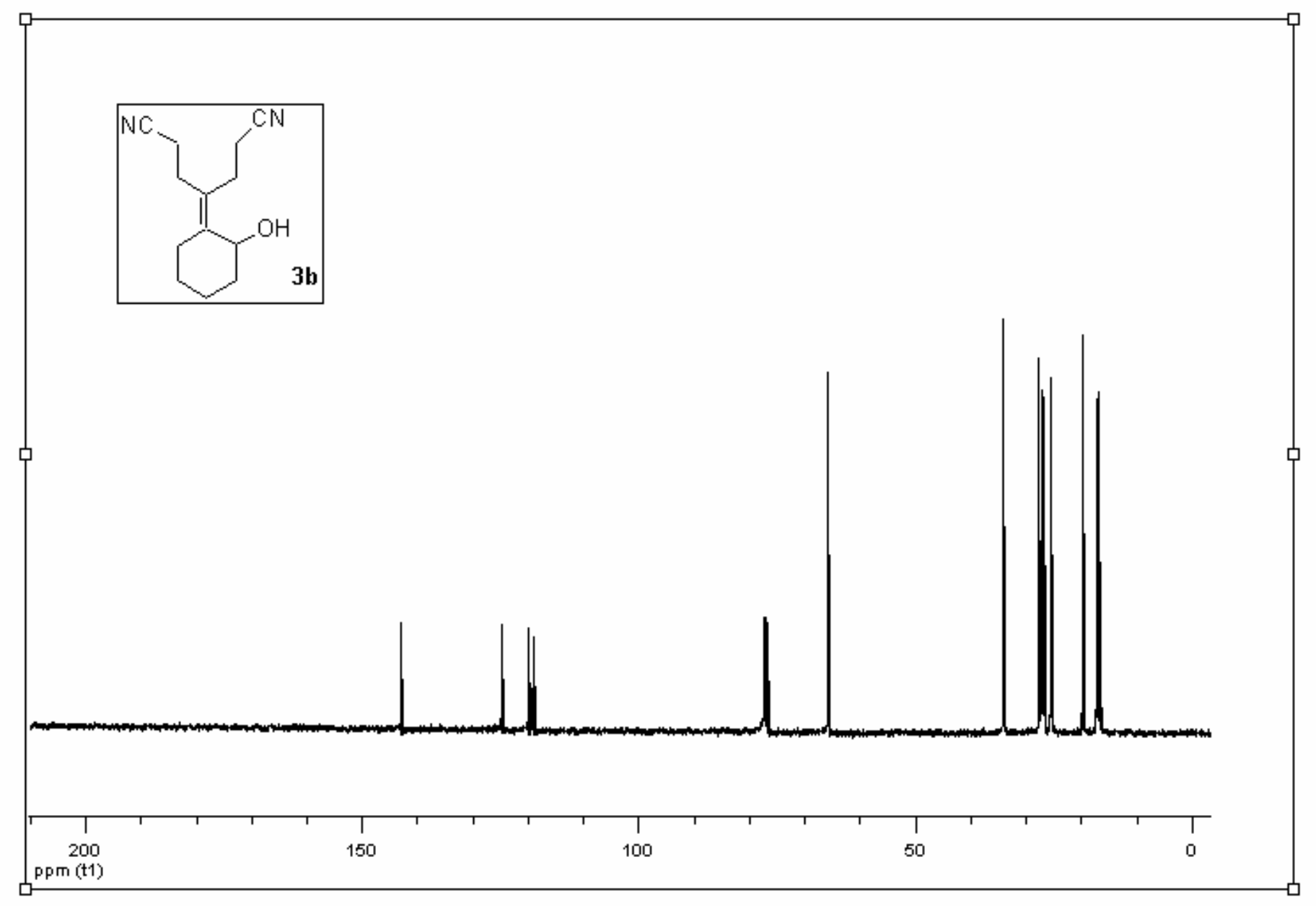

SI12 


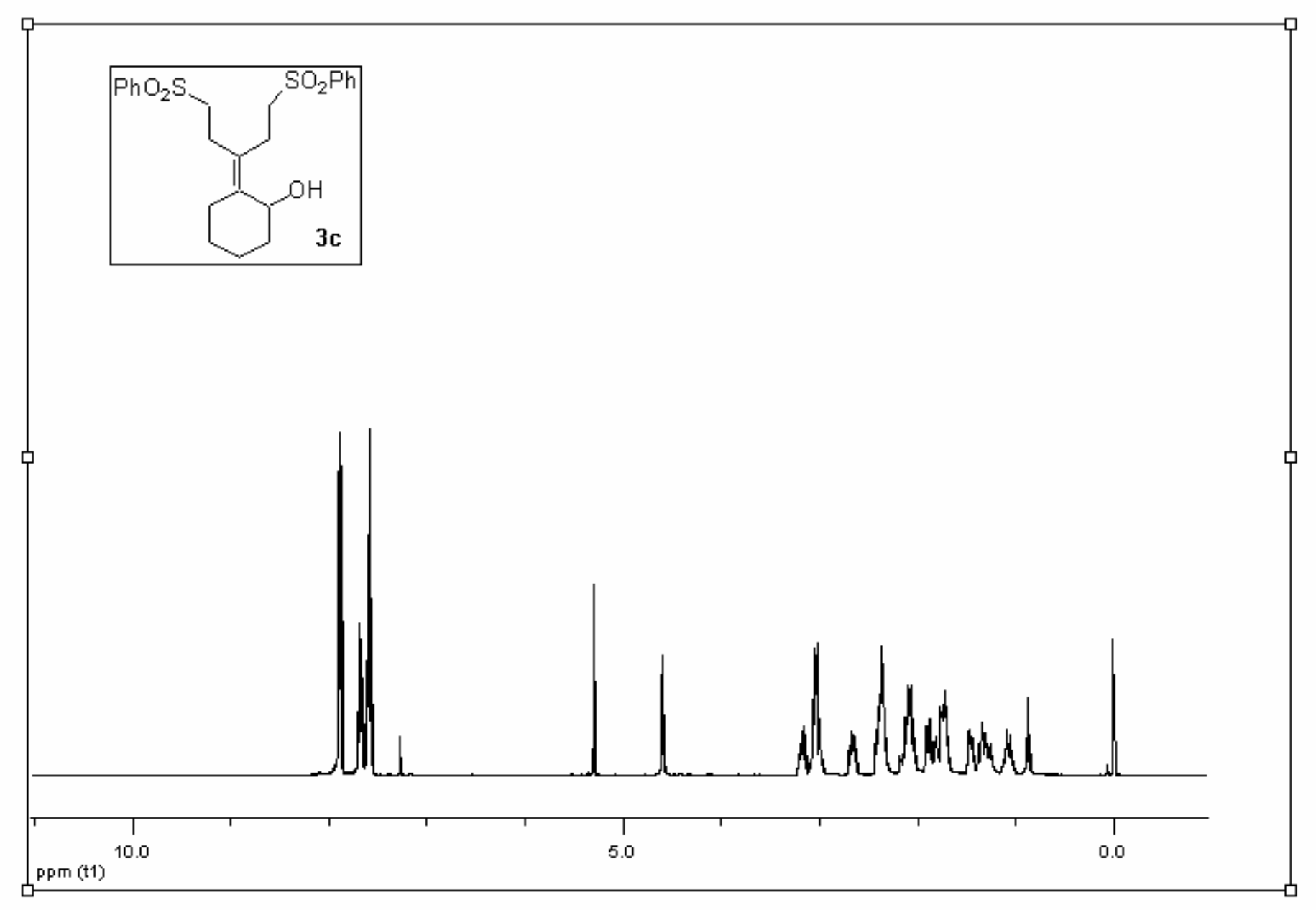

Sl13 


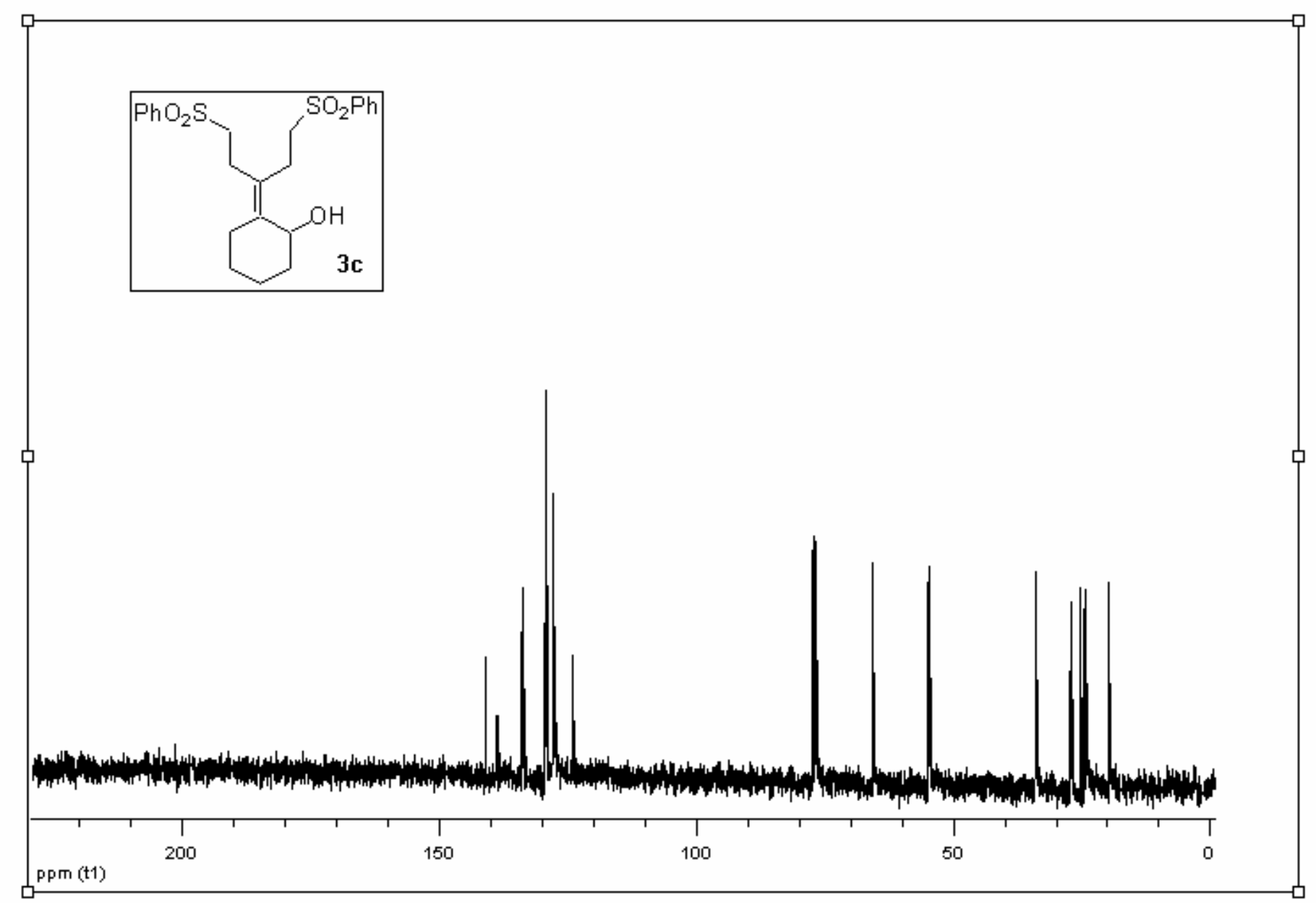

SI14 


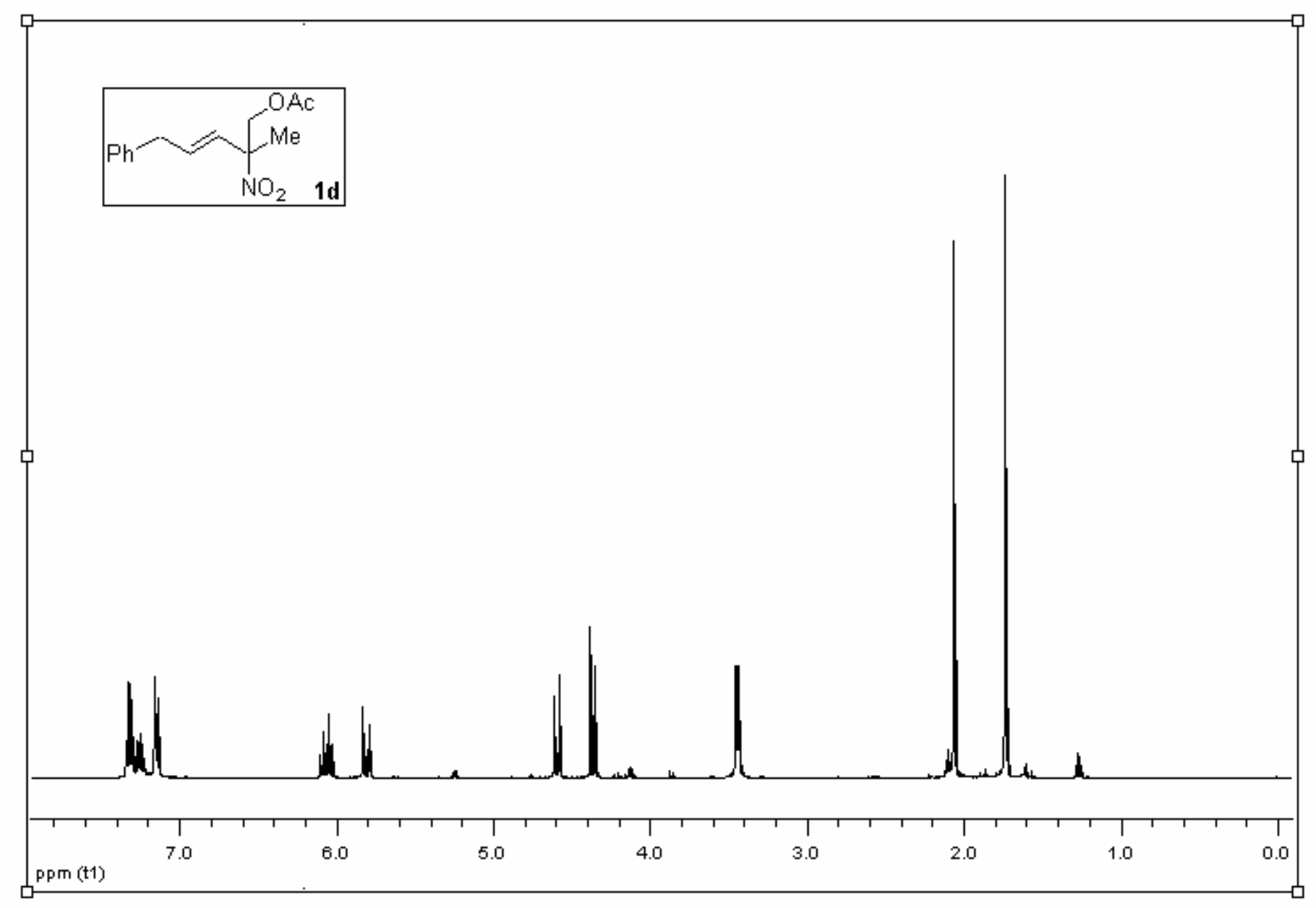




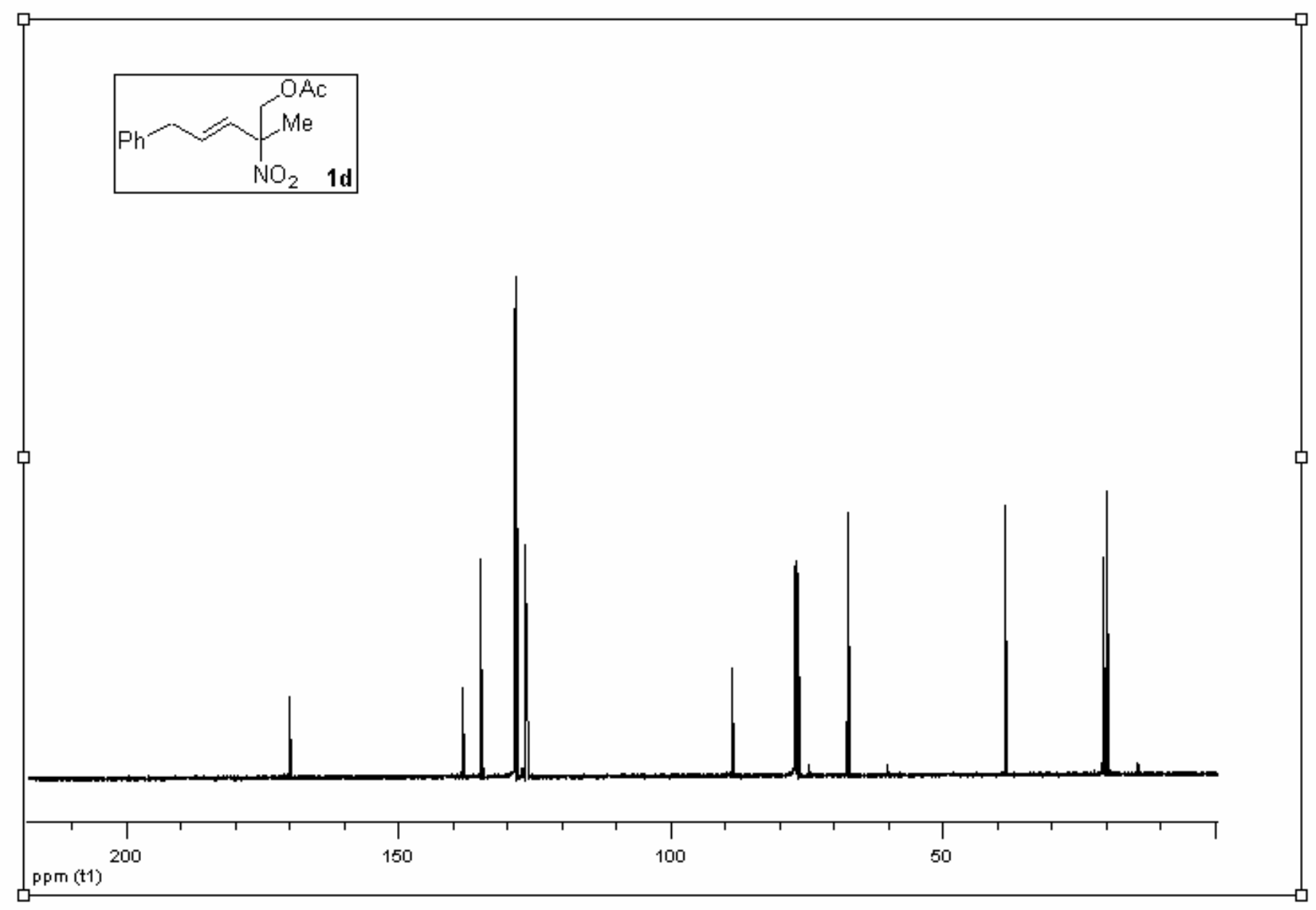

SI16 


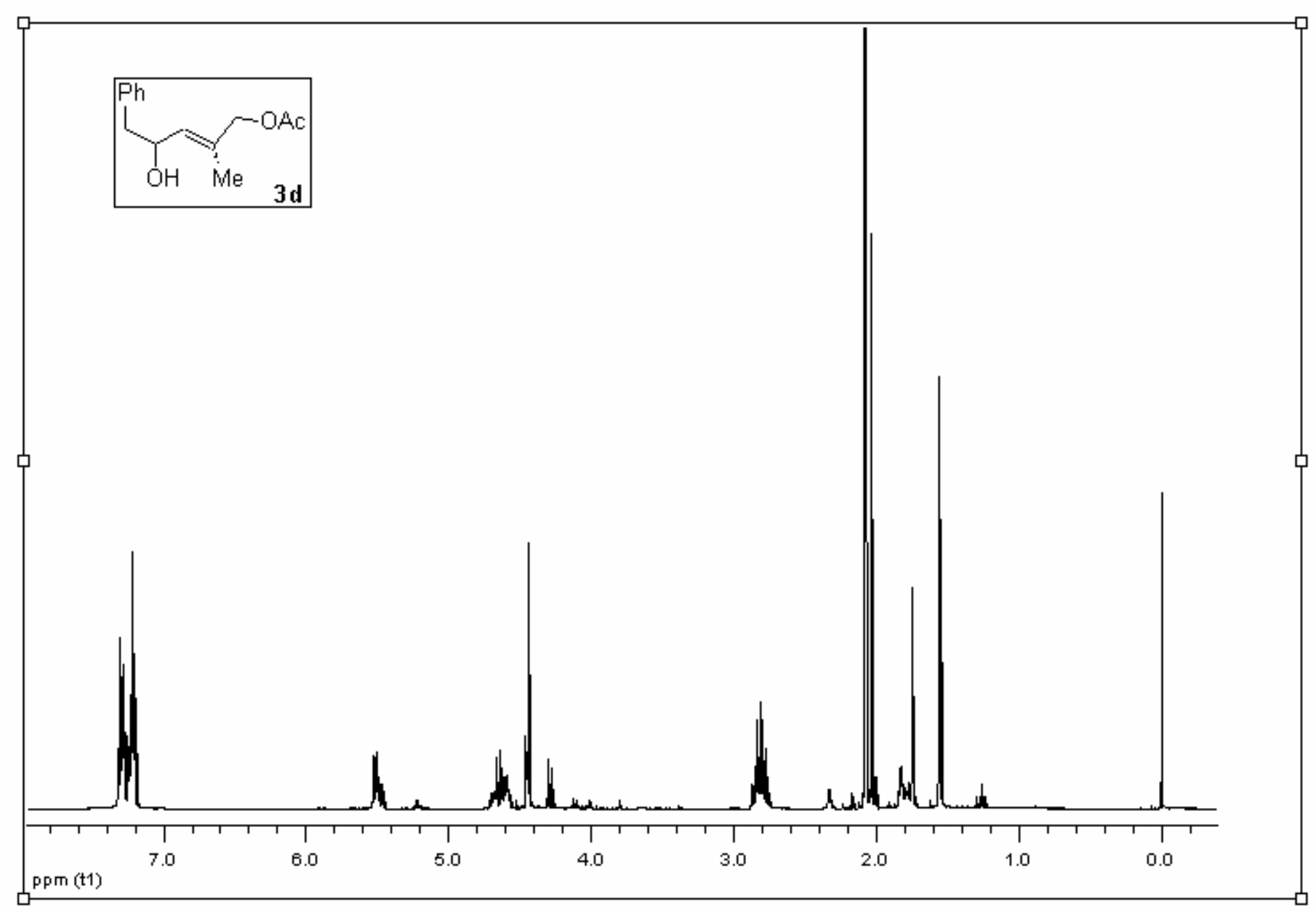




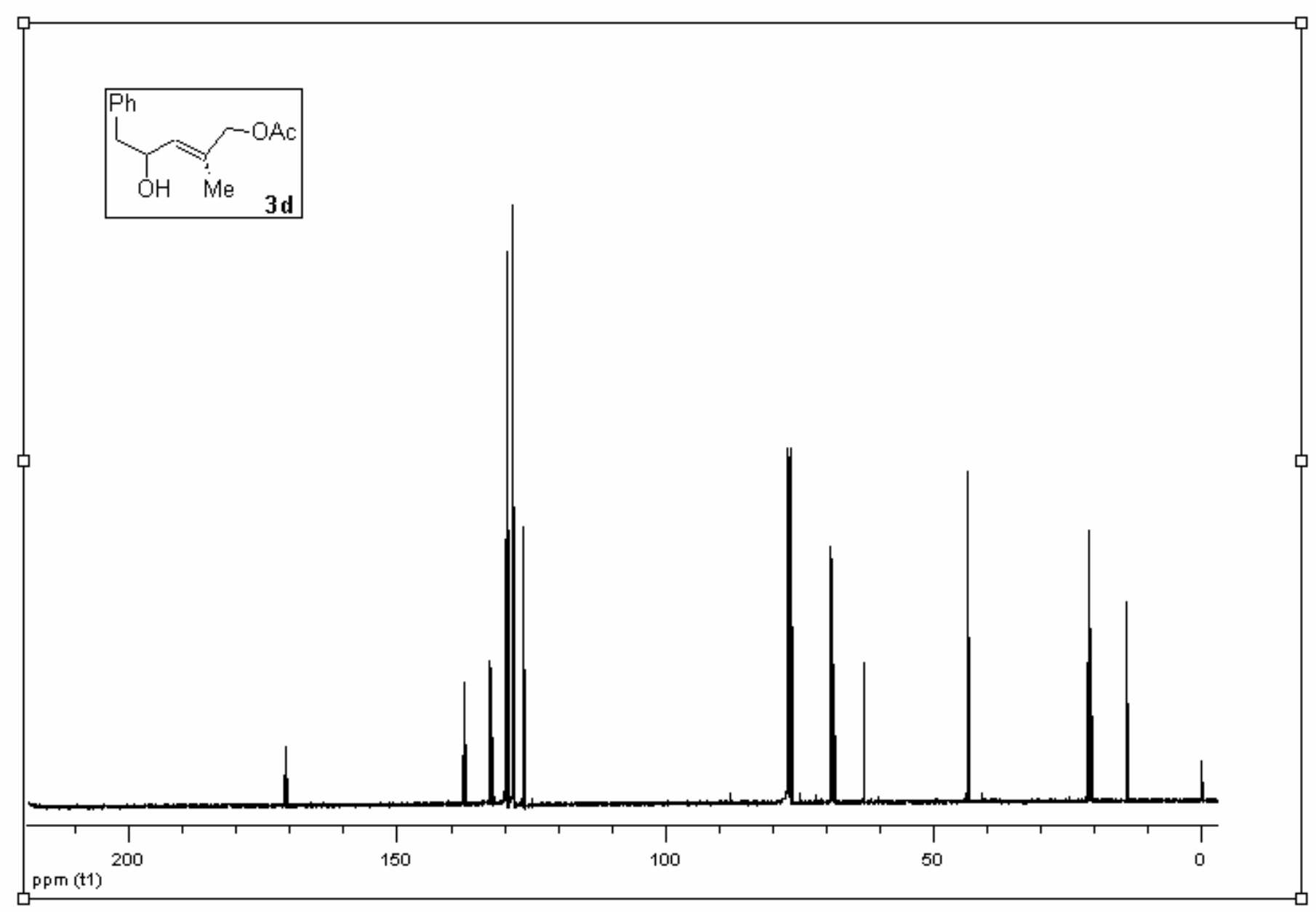

SI18 


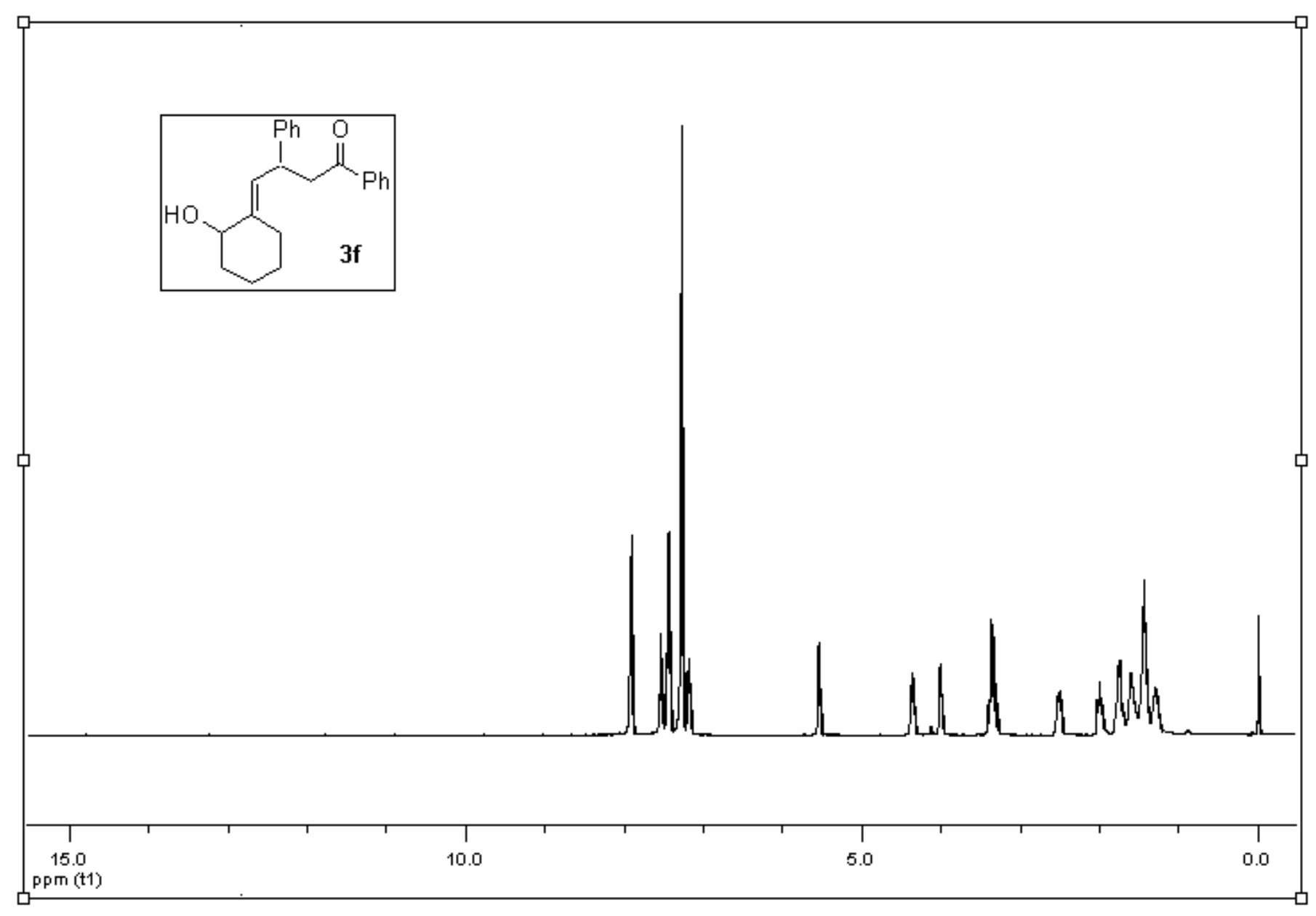

SI19 


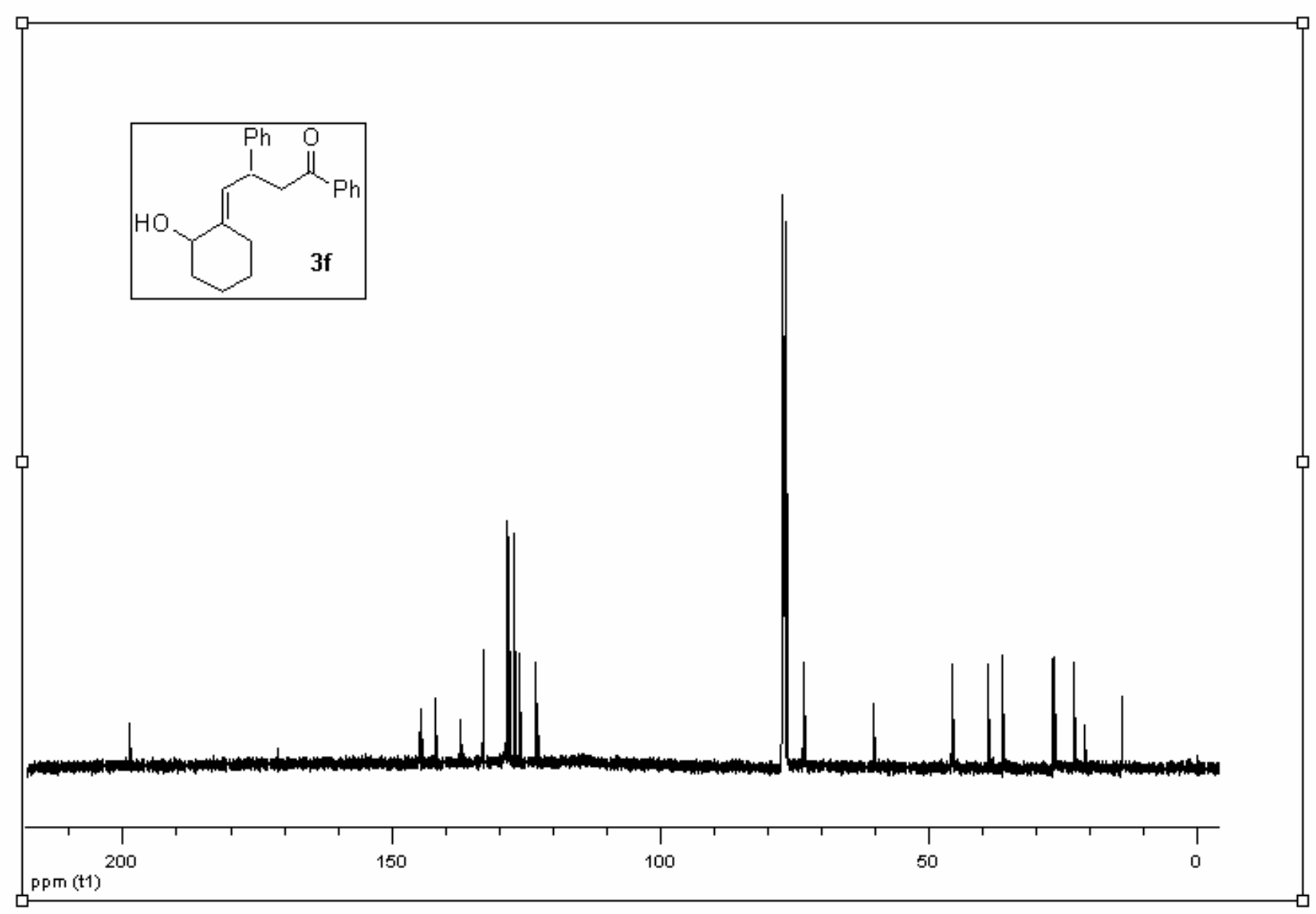




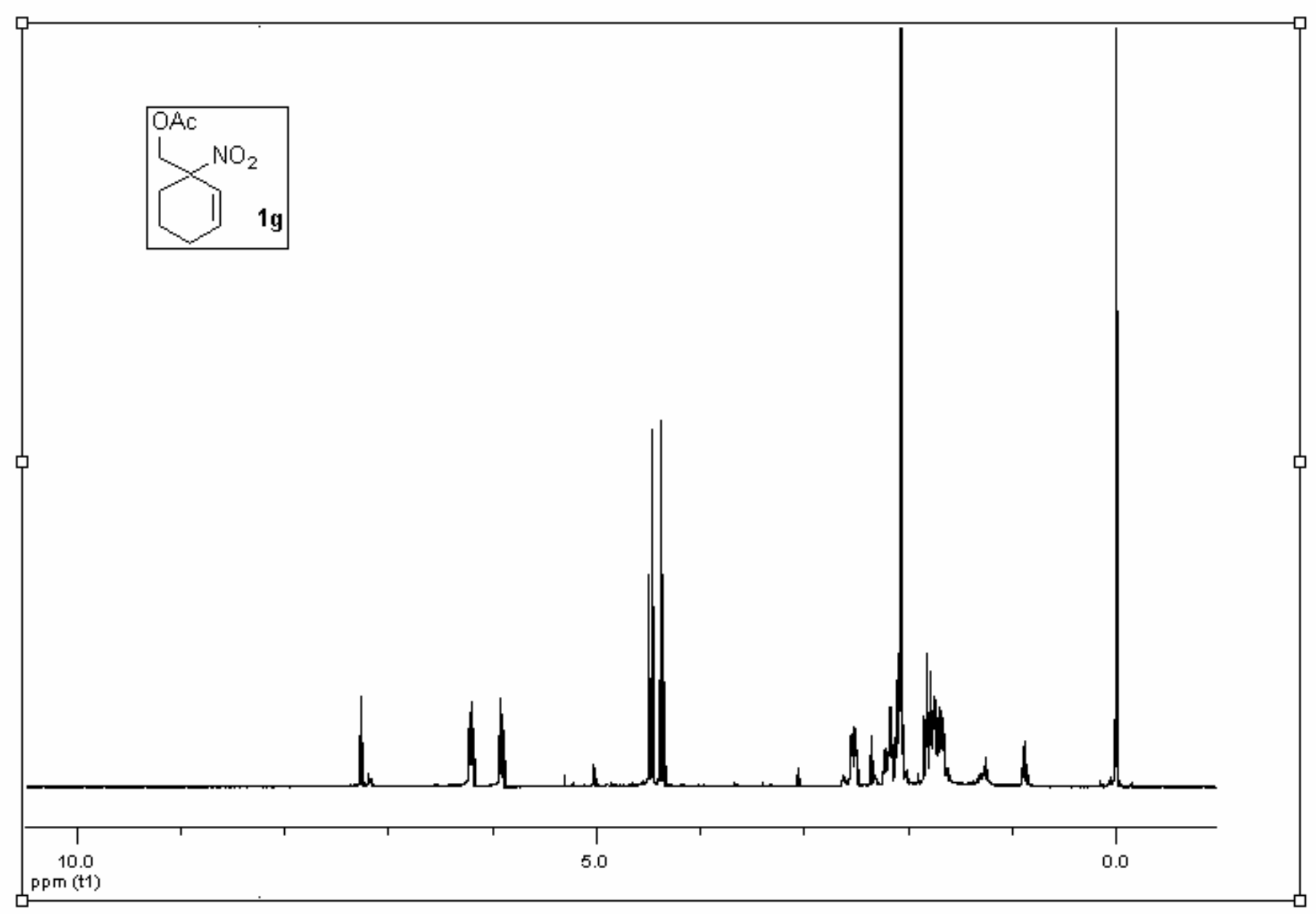




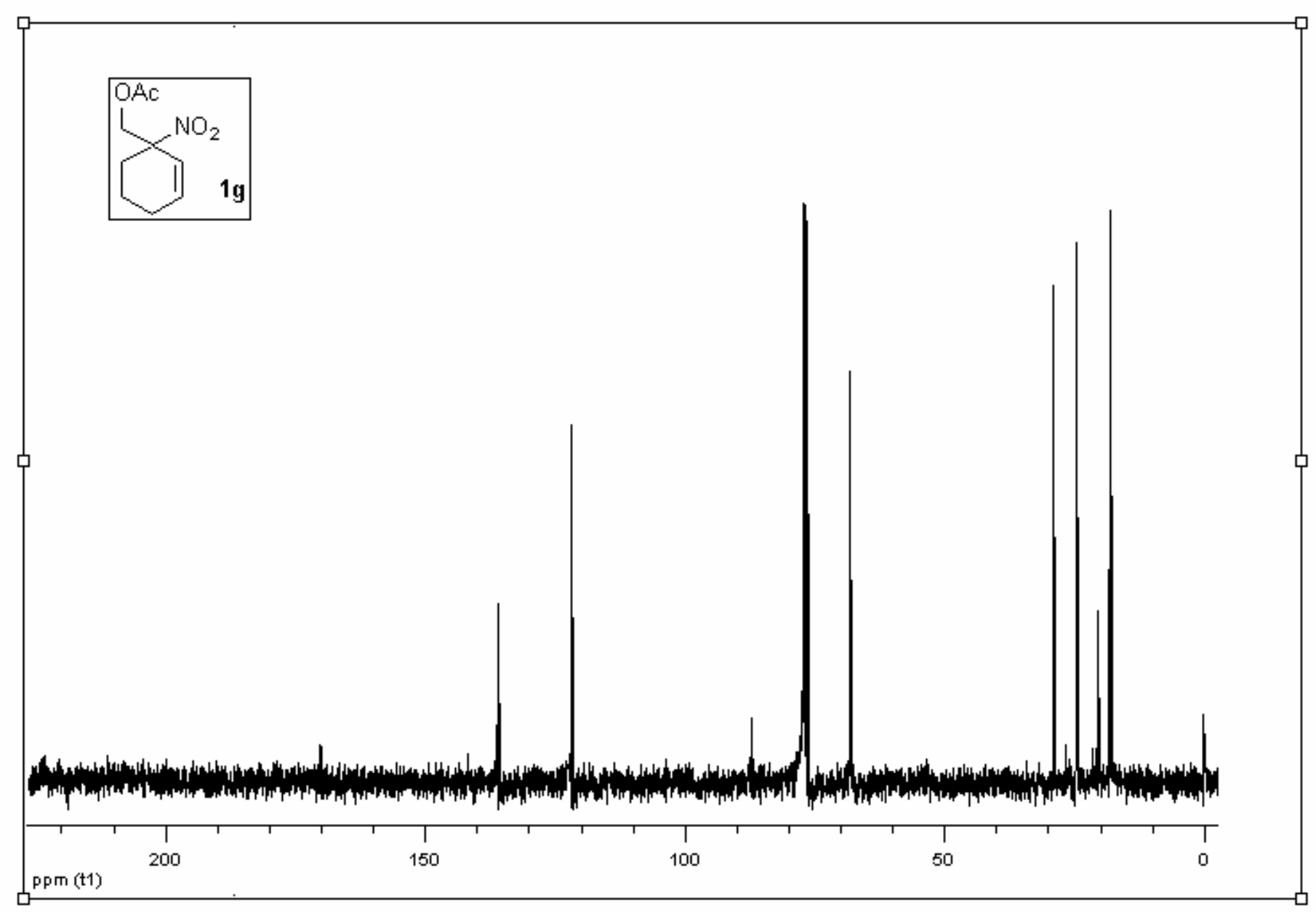




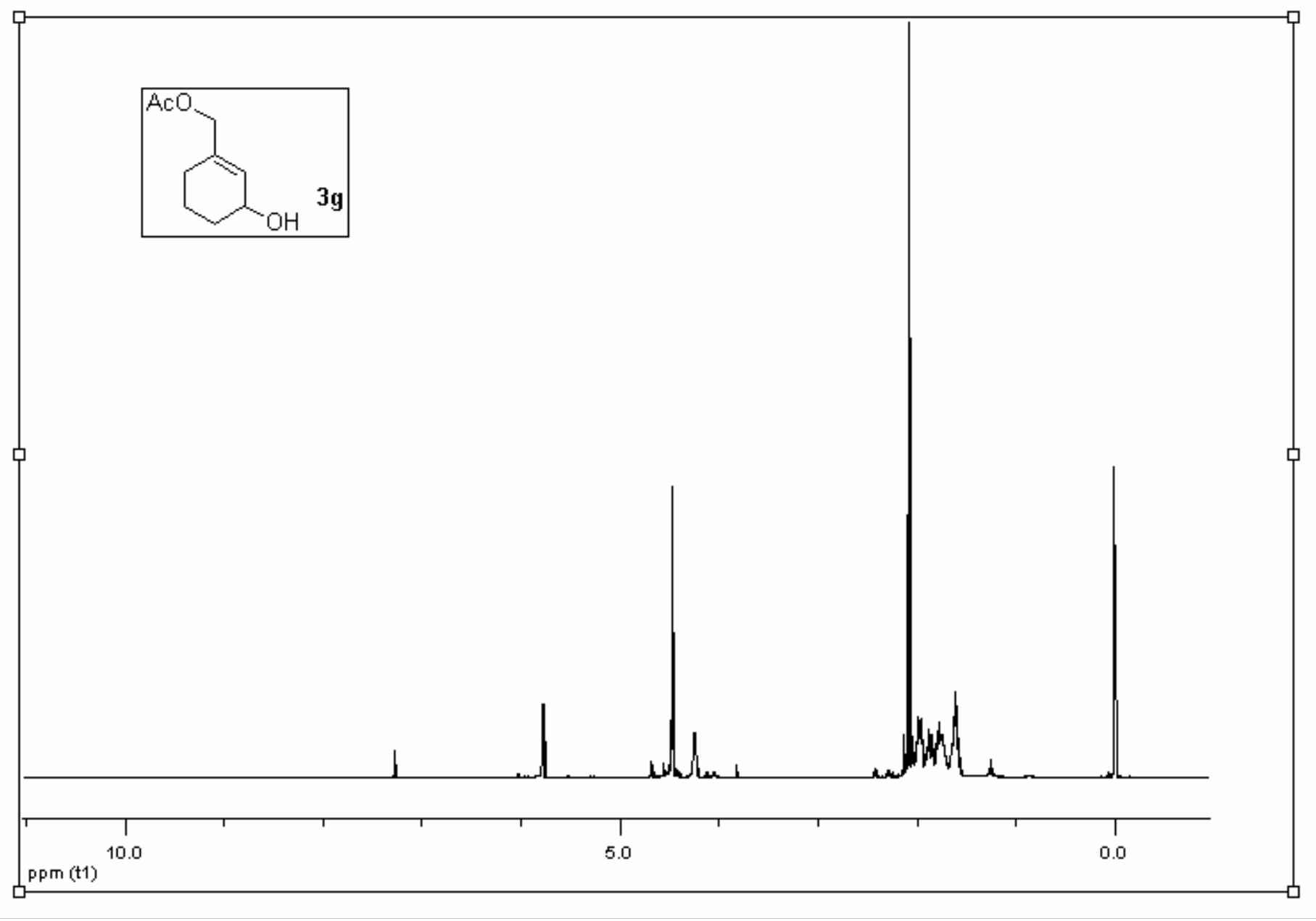

SI23 


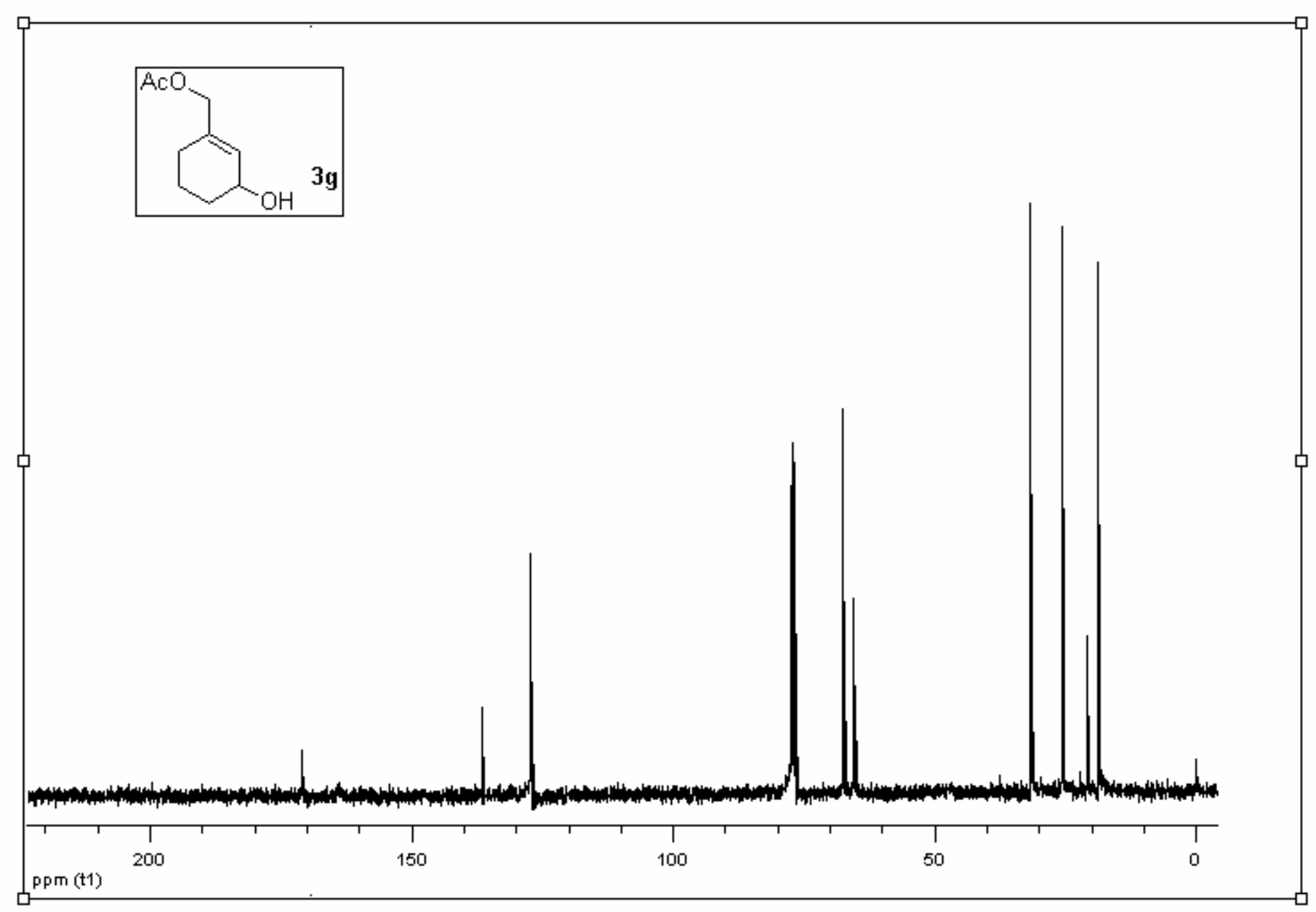




$$
\text { I }
$$




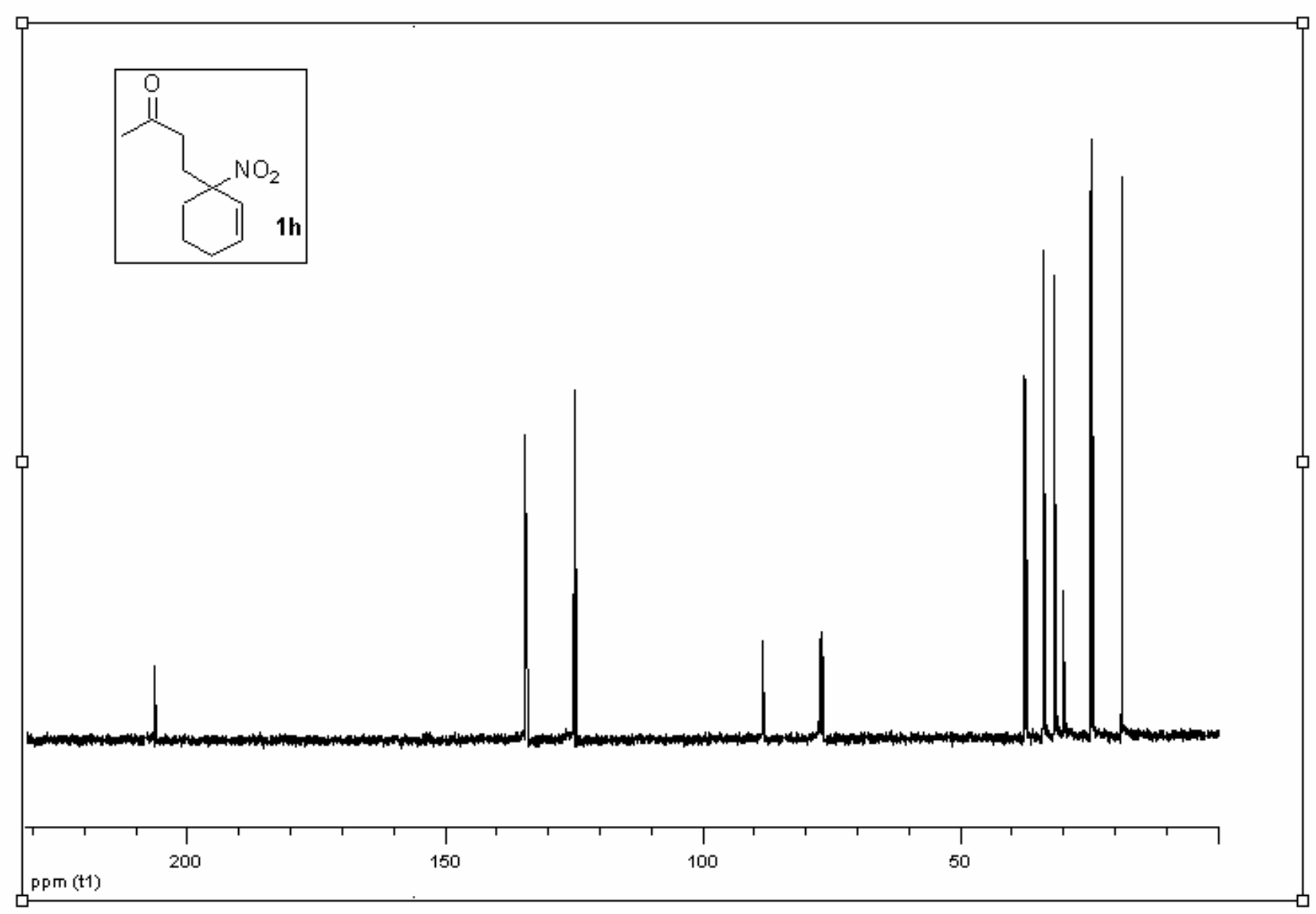




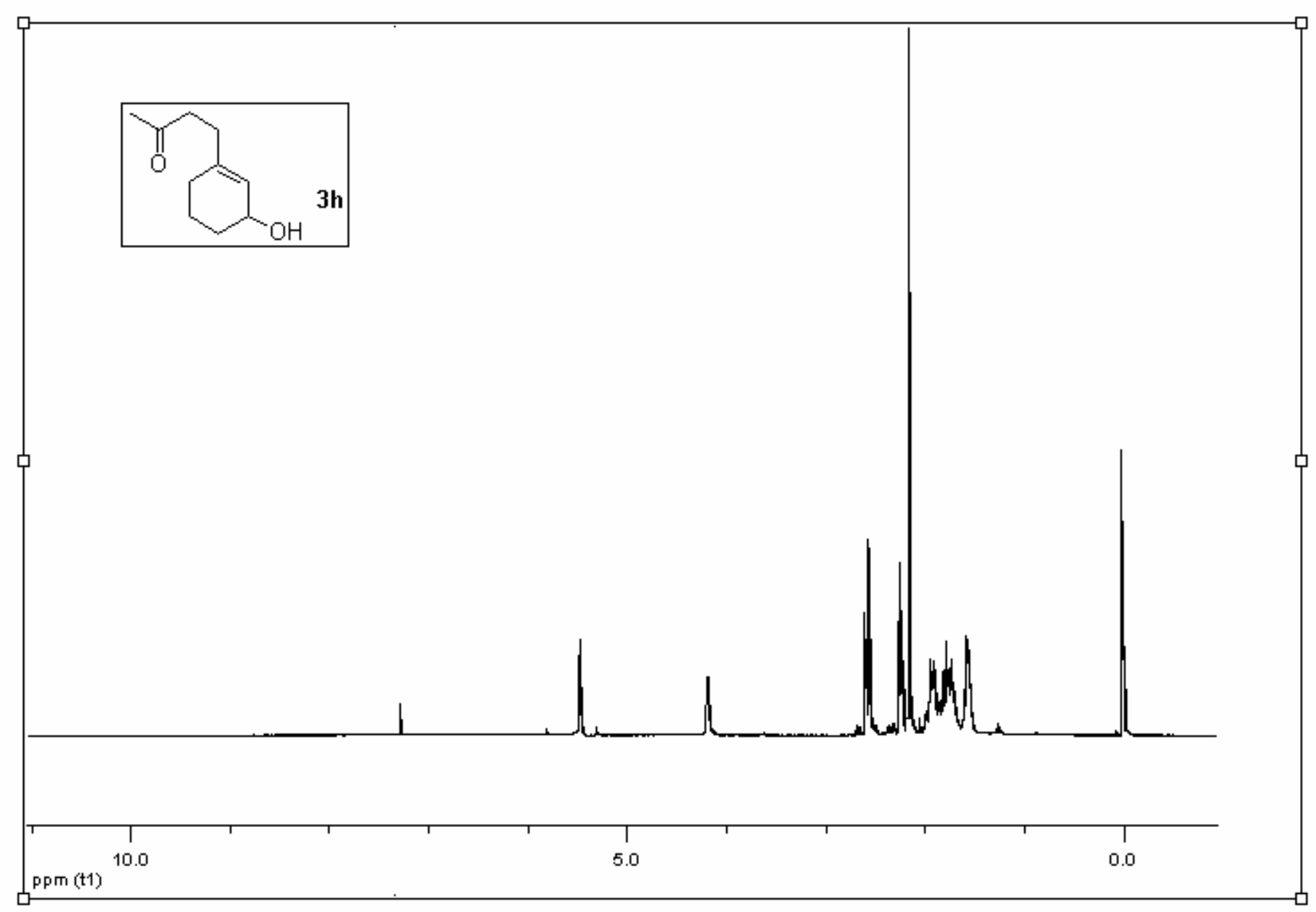




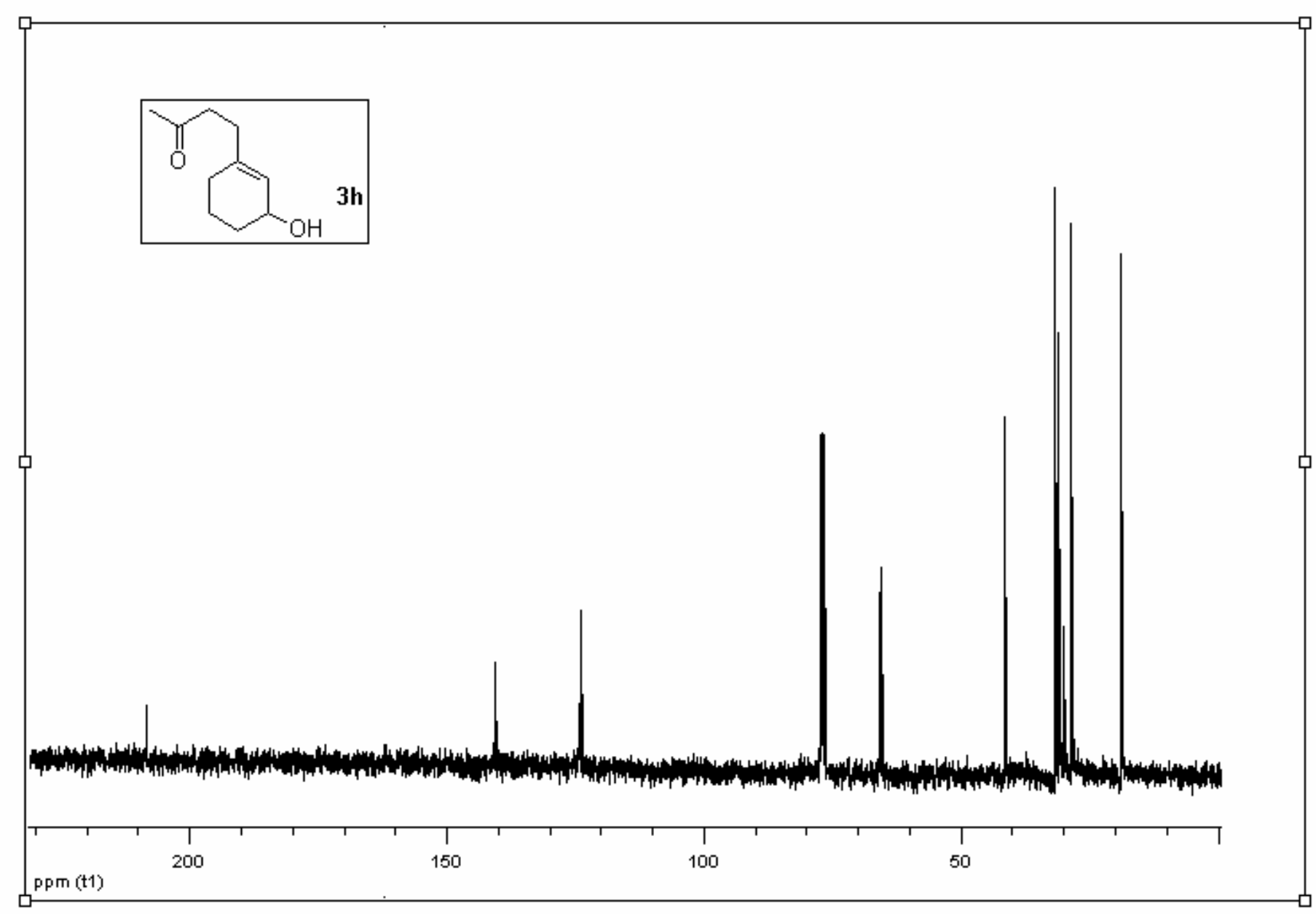




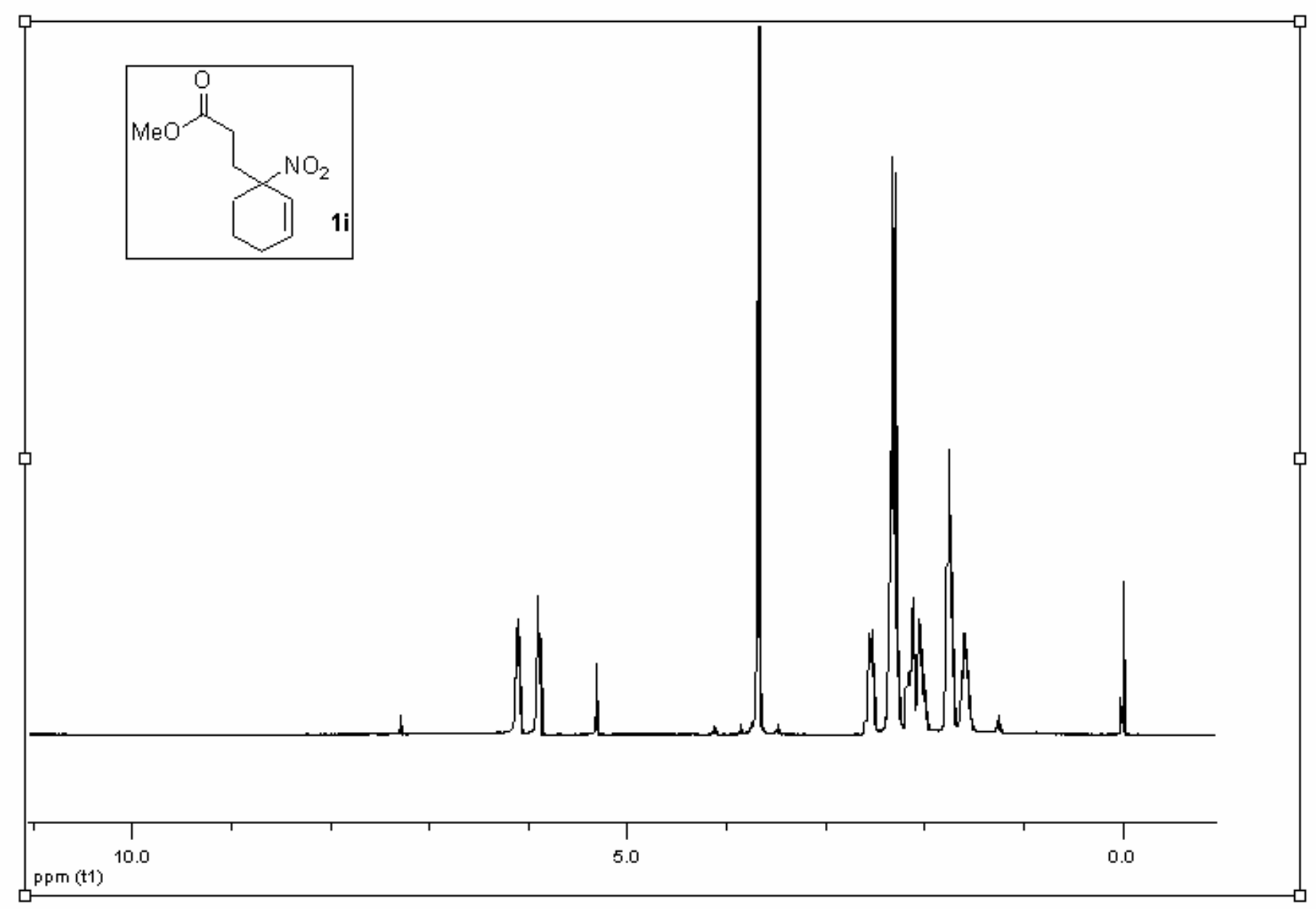

SI29 


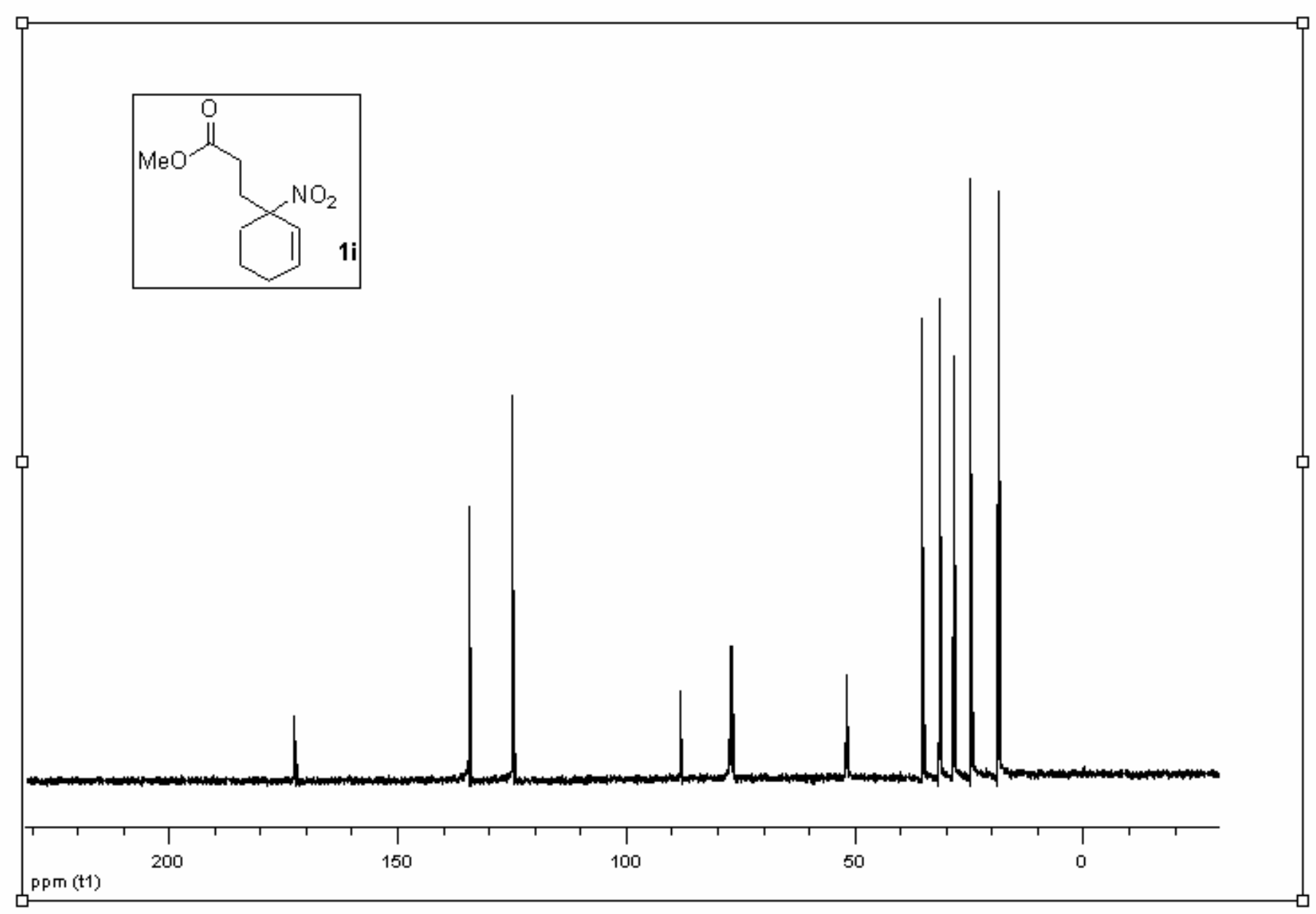




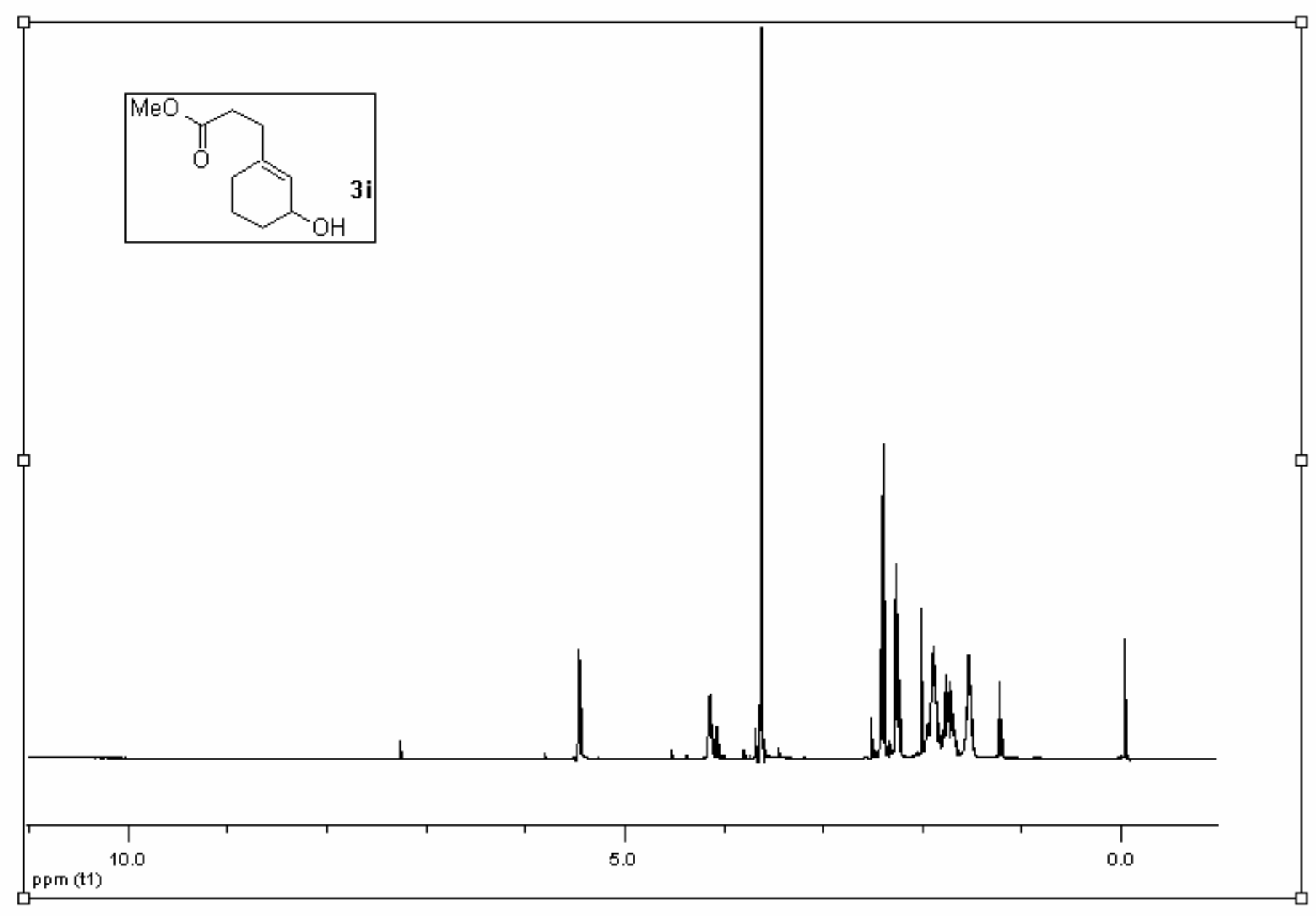




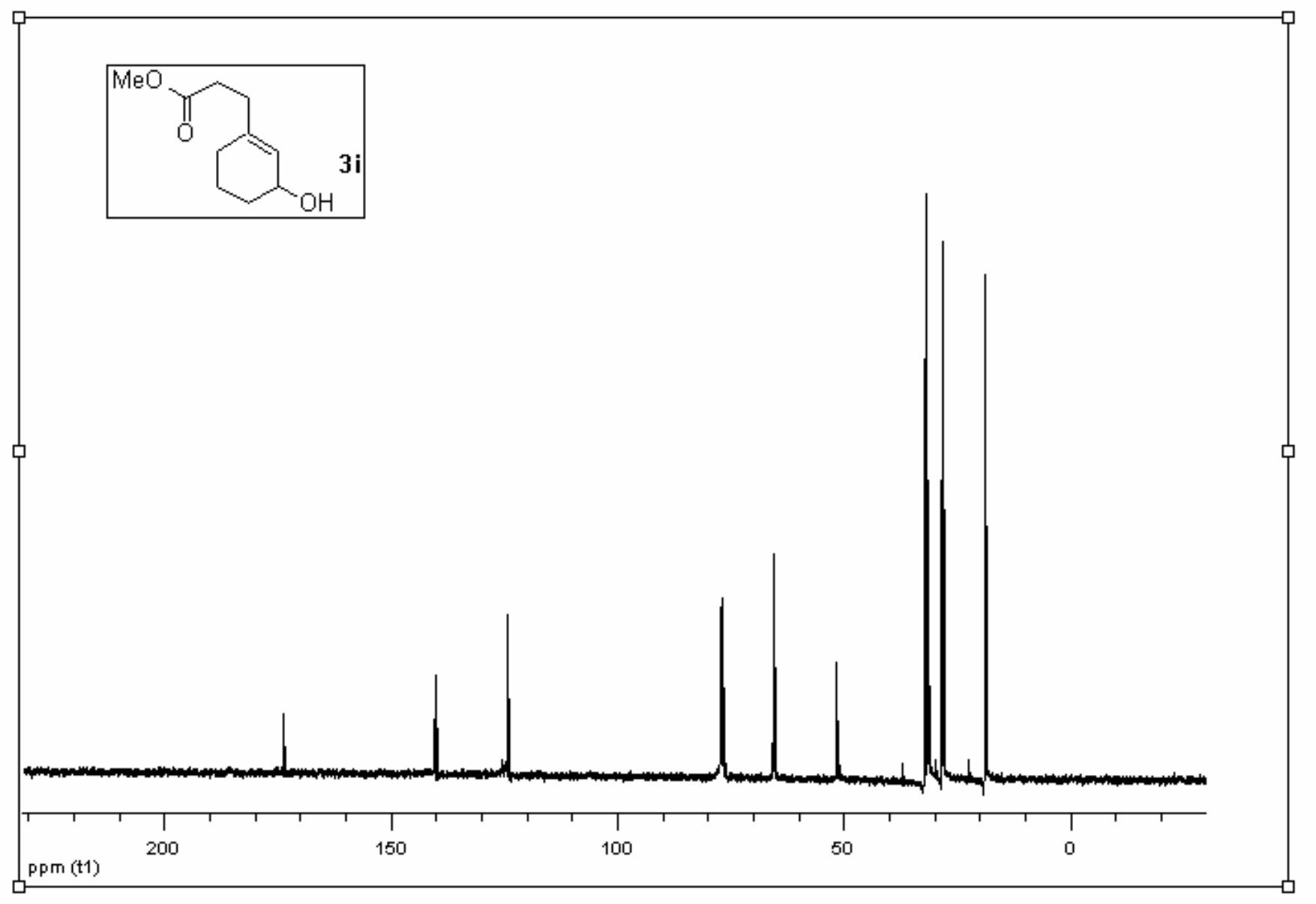

SI32 


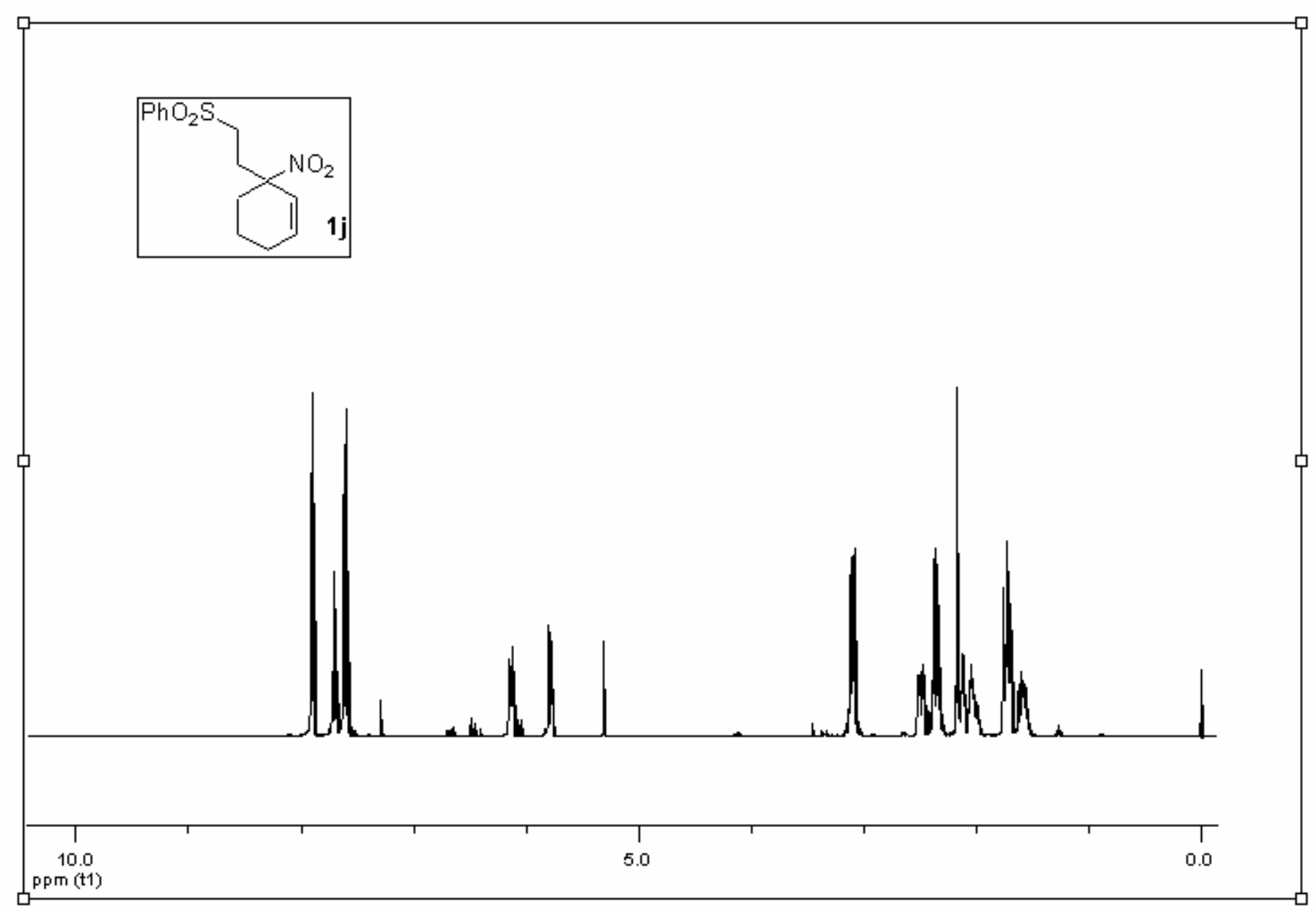




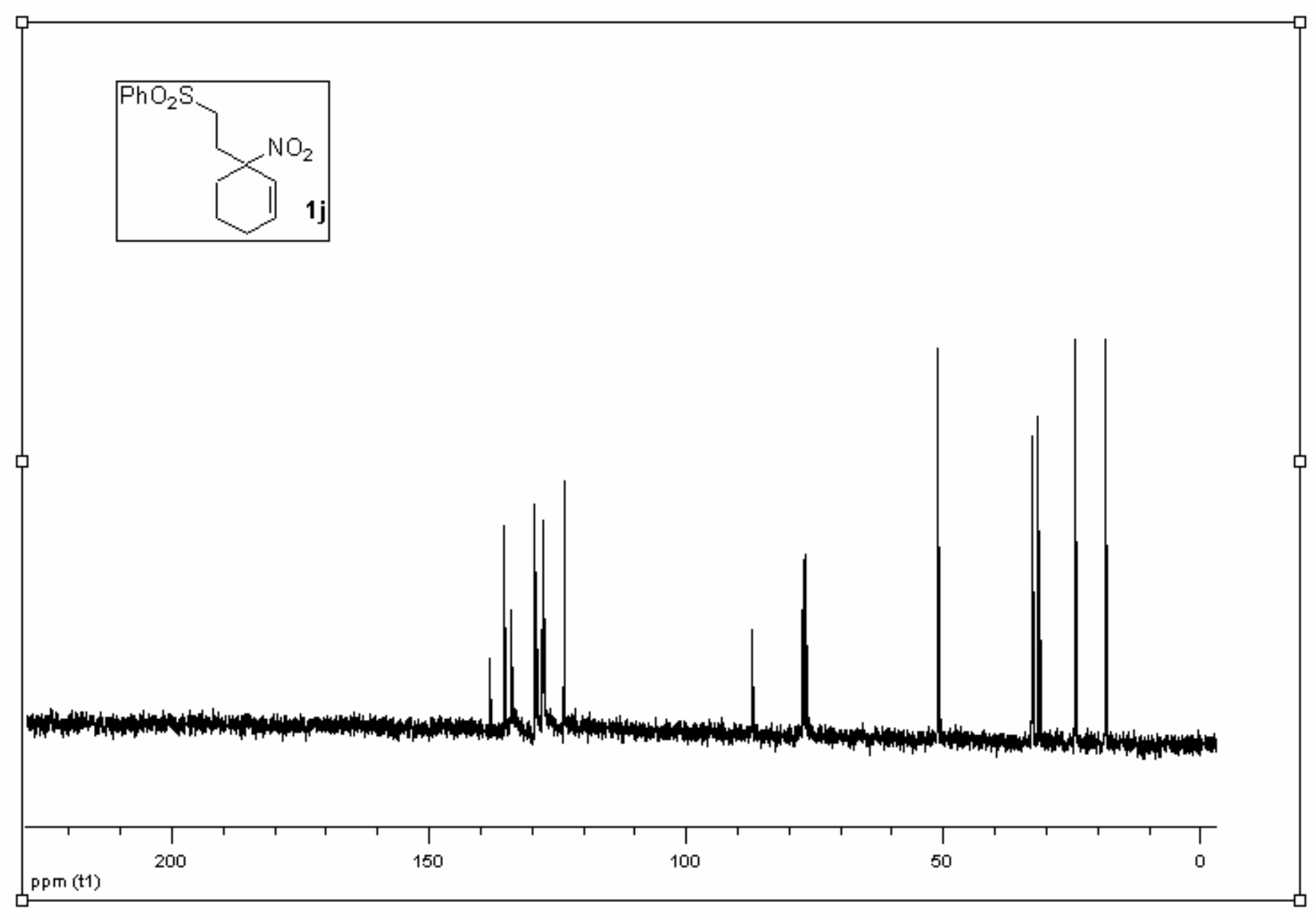




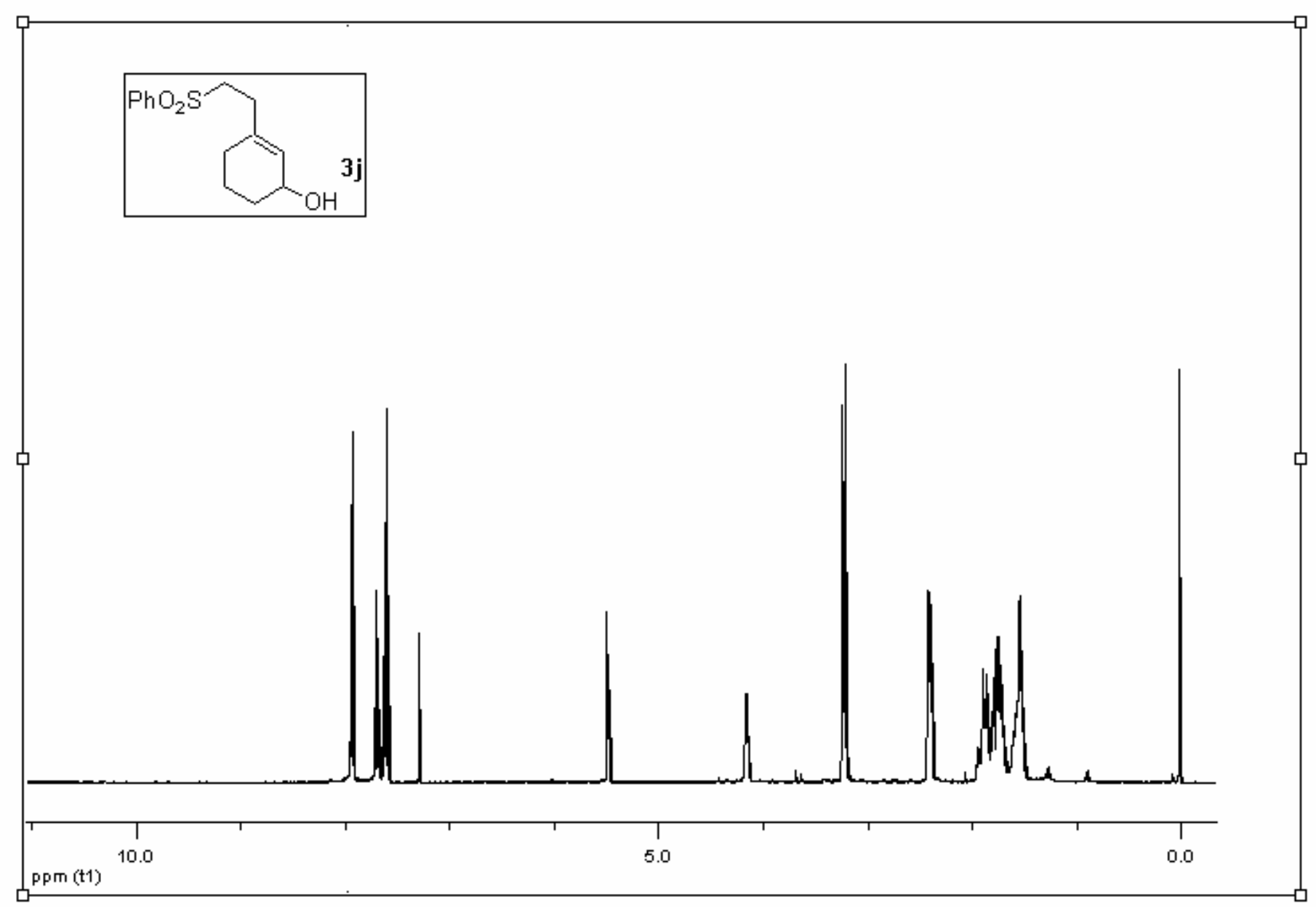




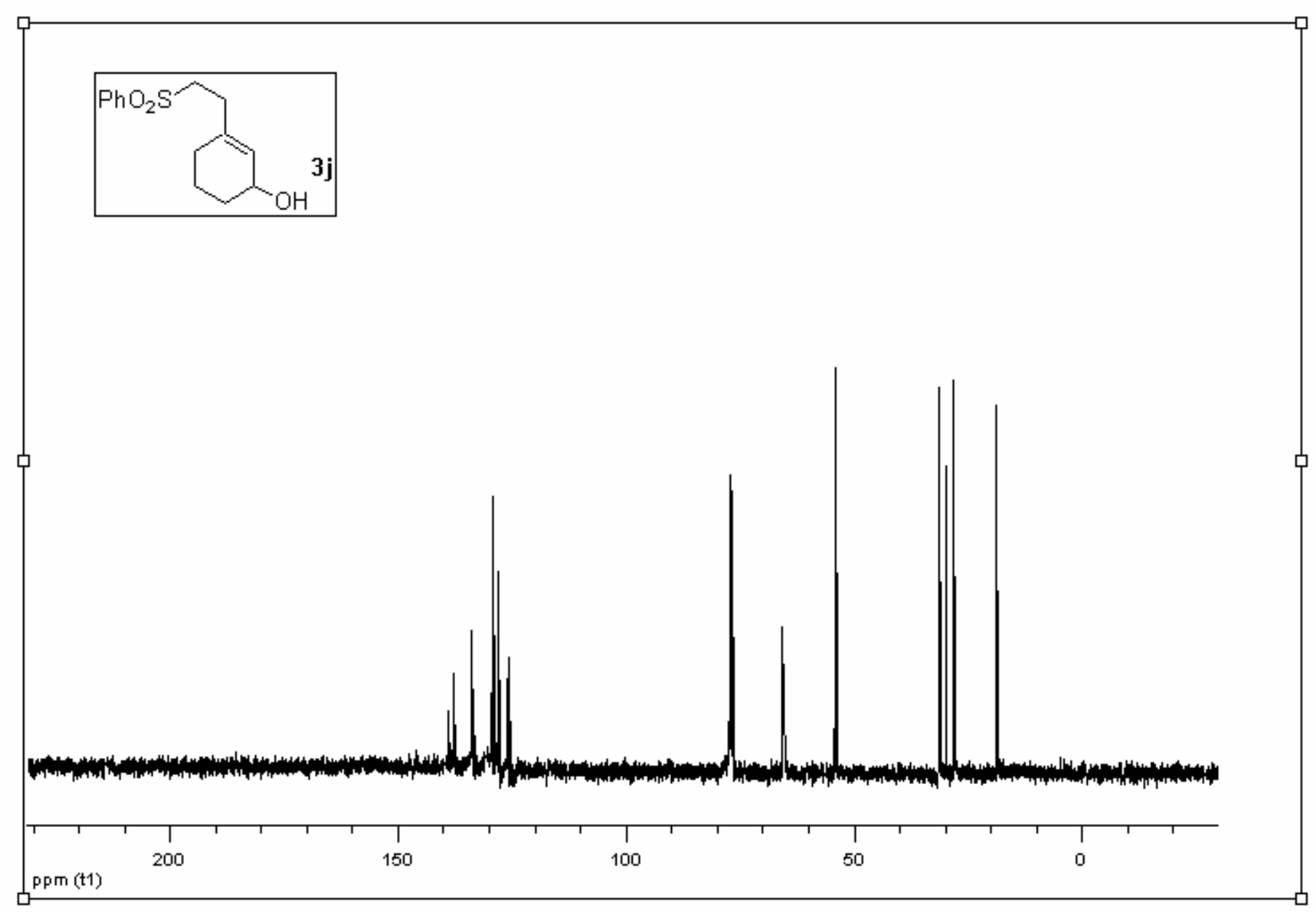




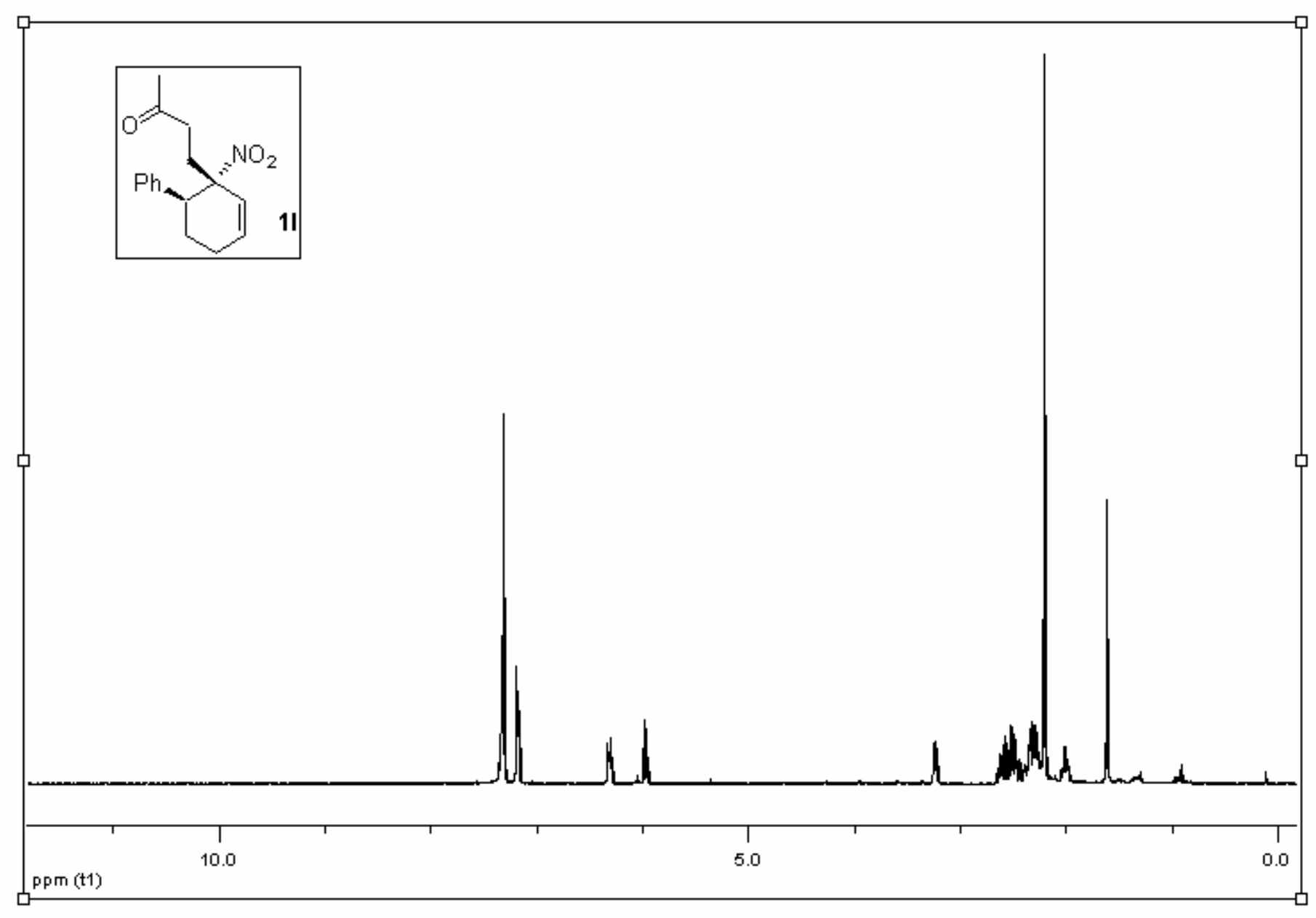




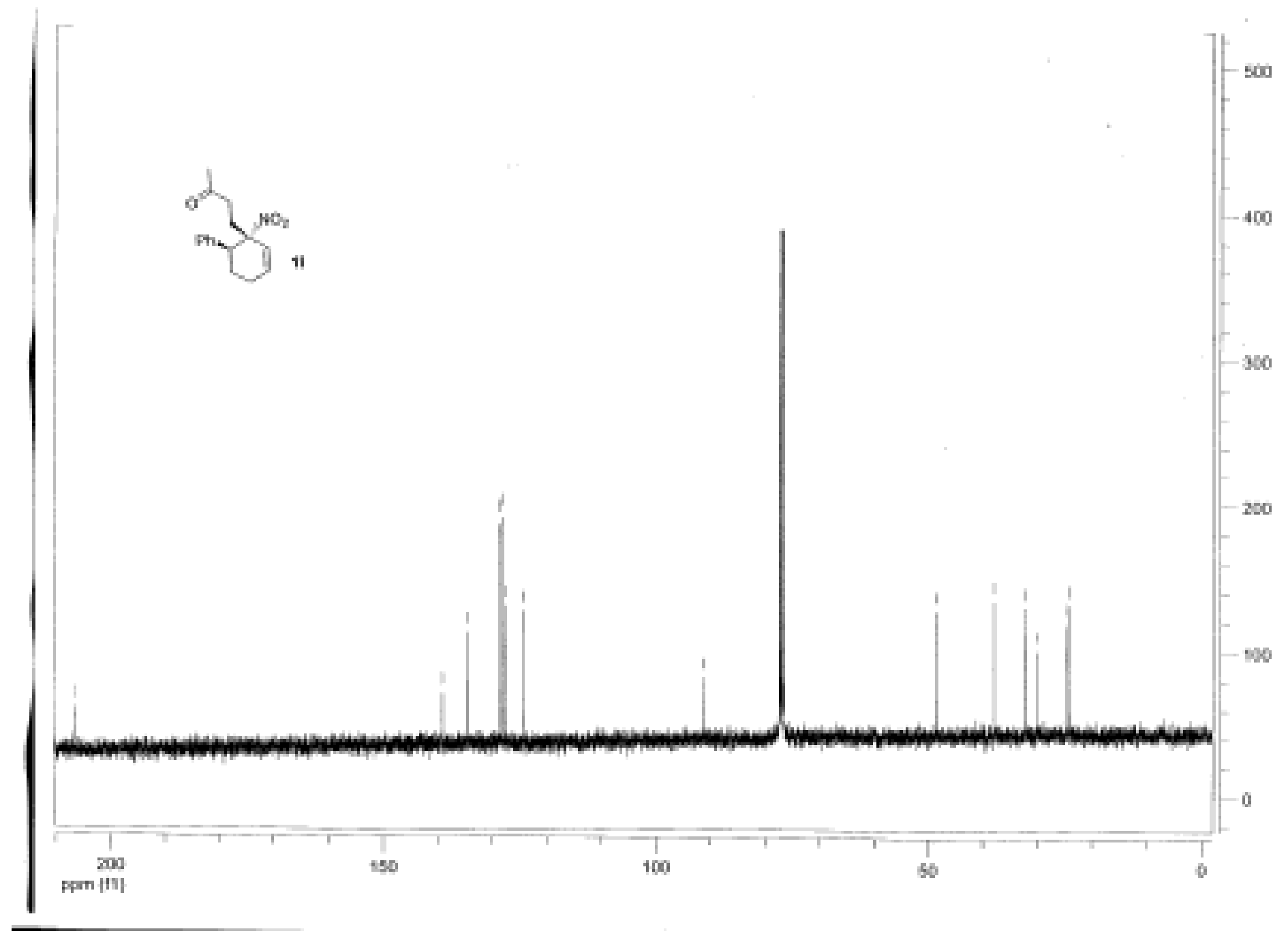




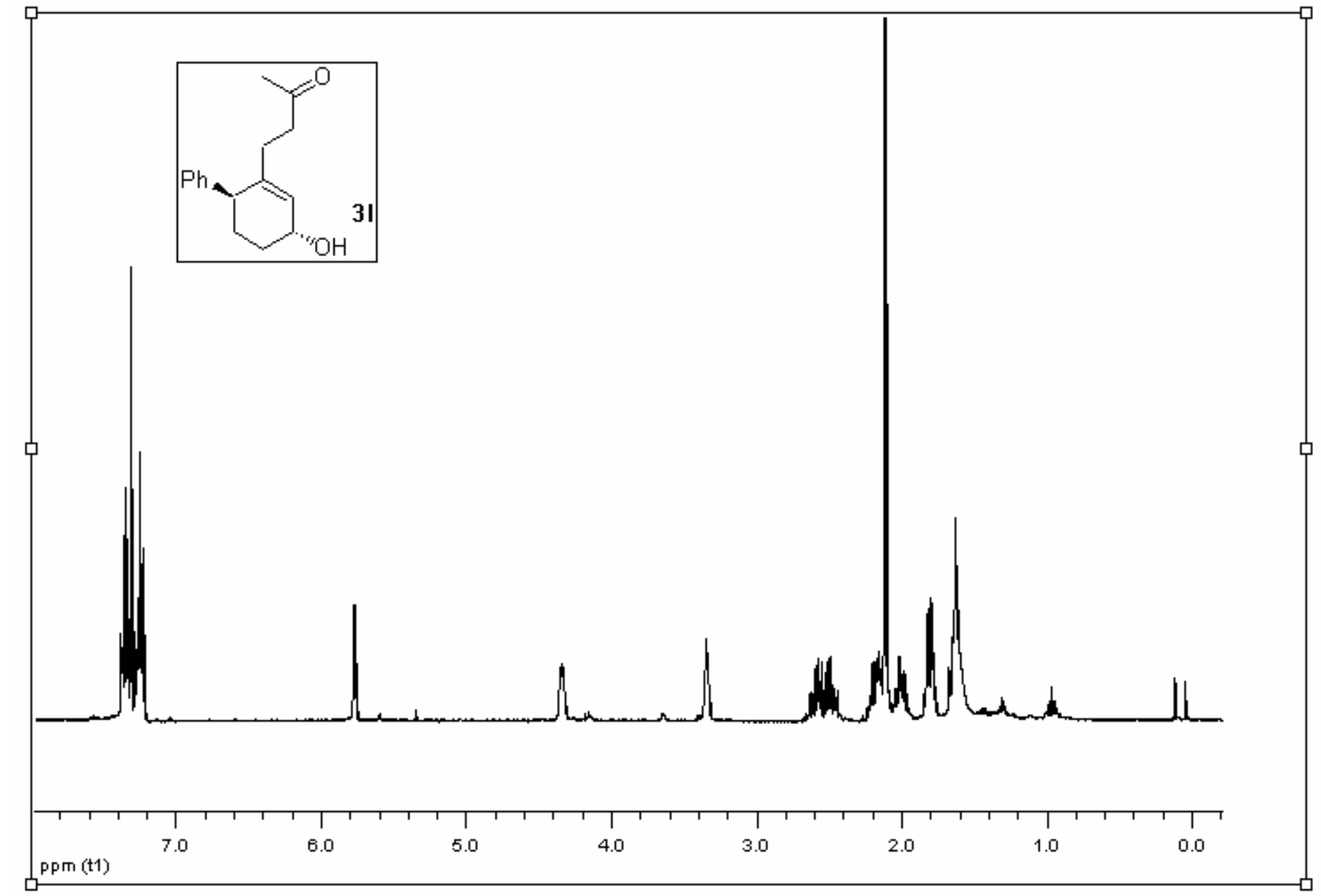




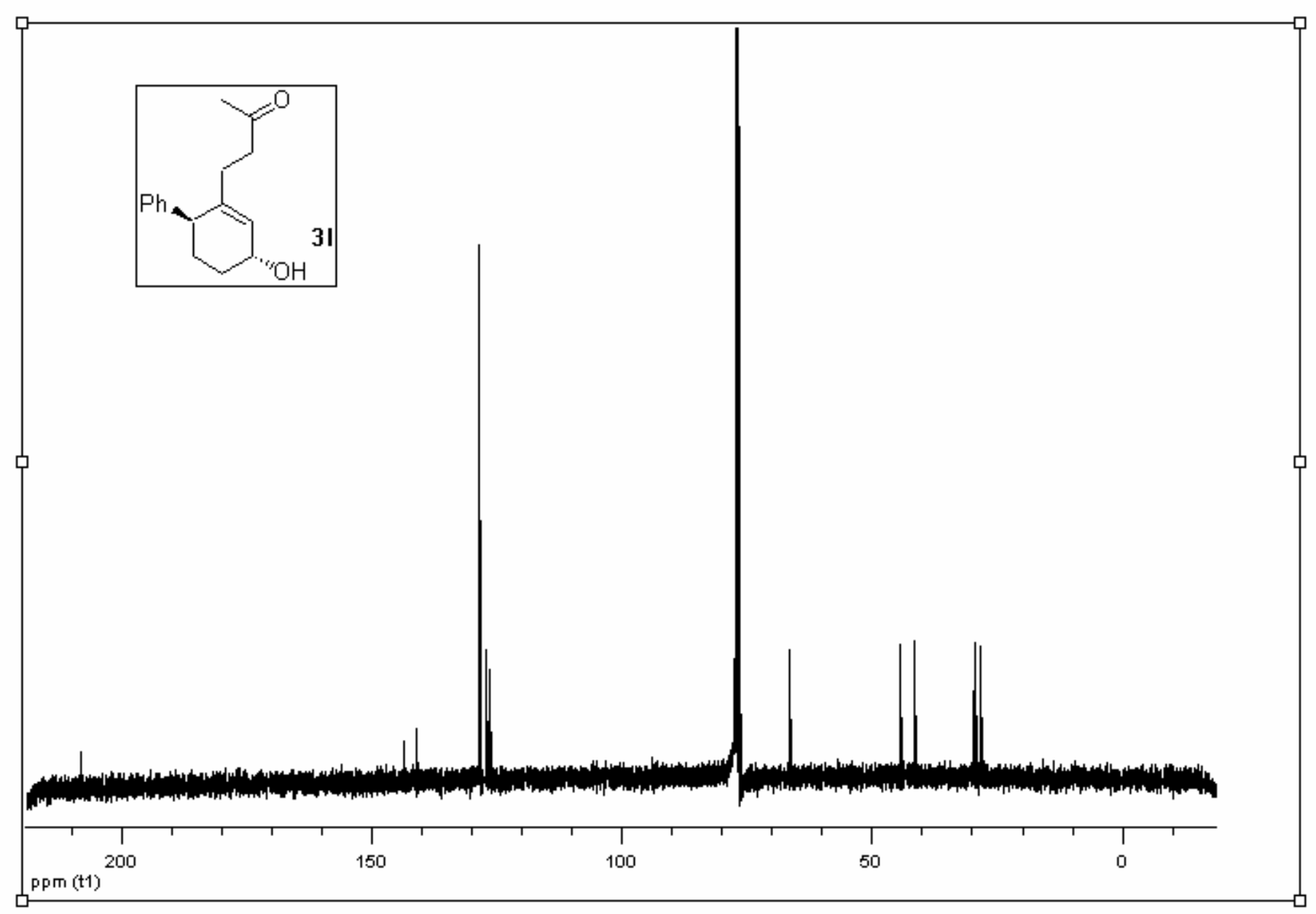

SI40 


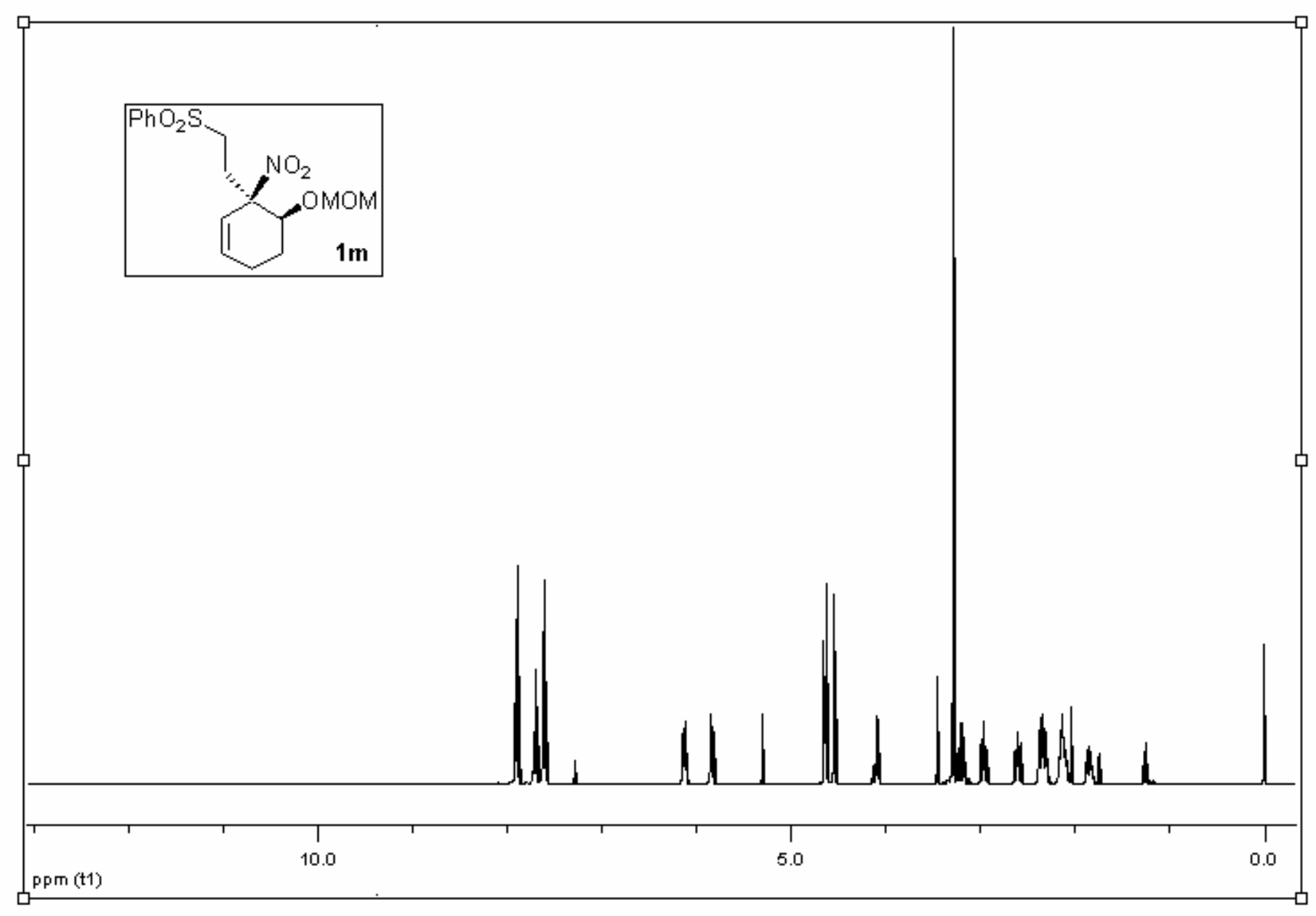




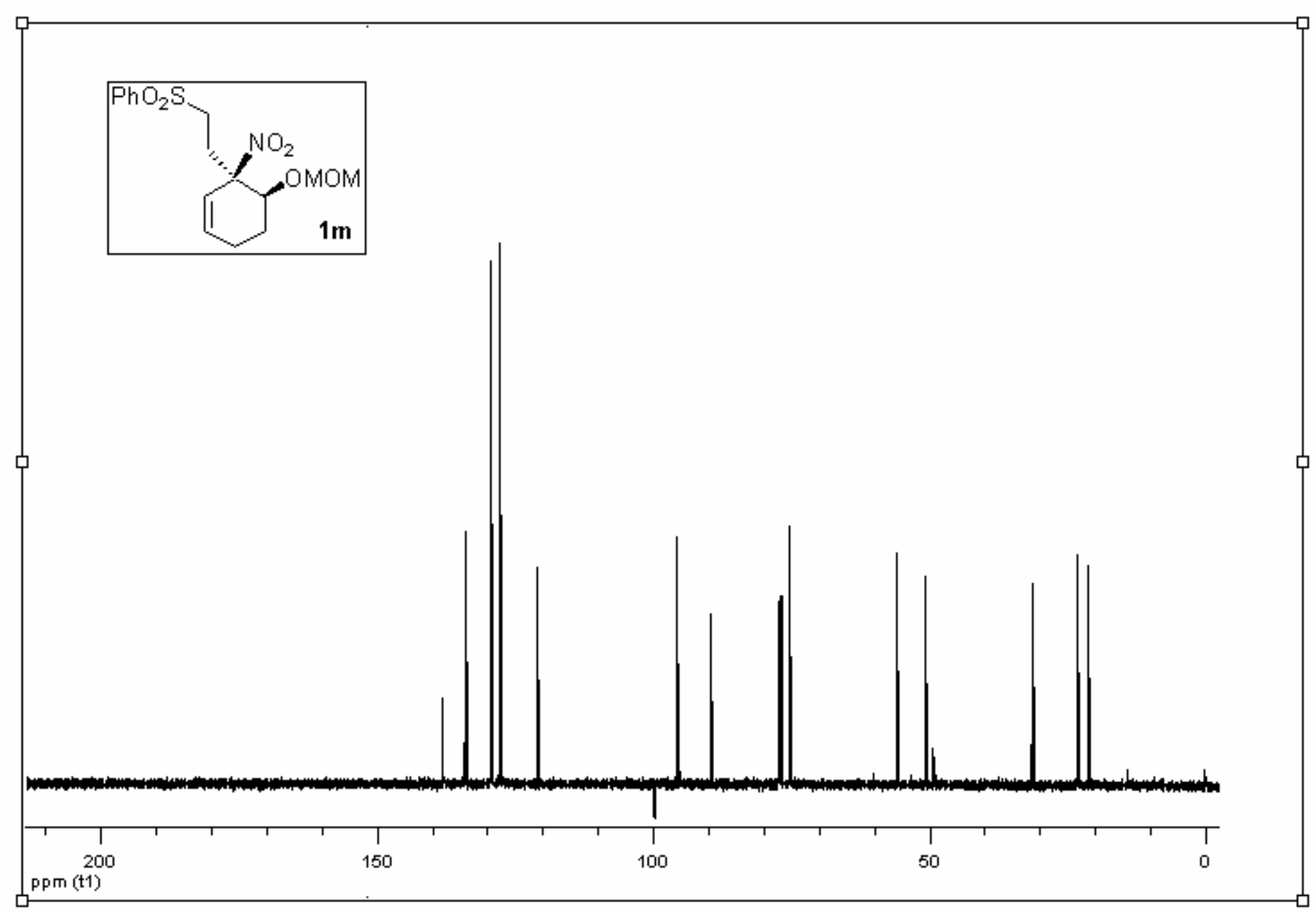




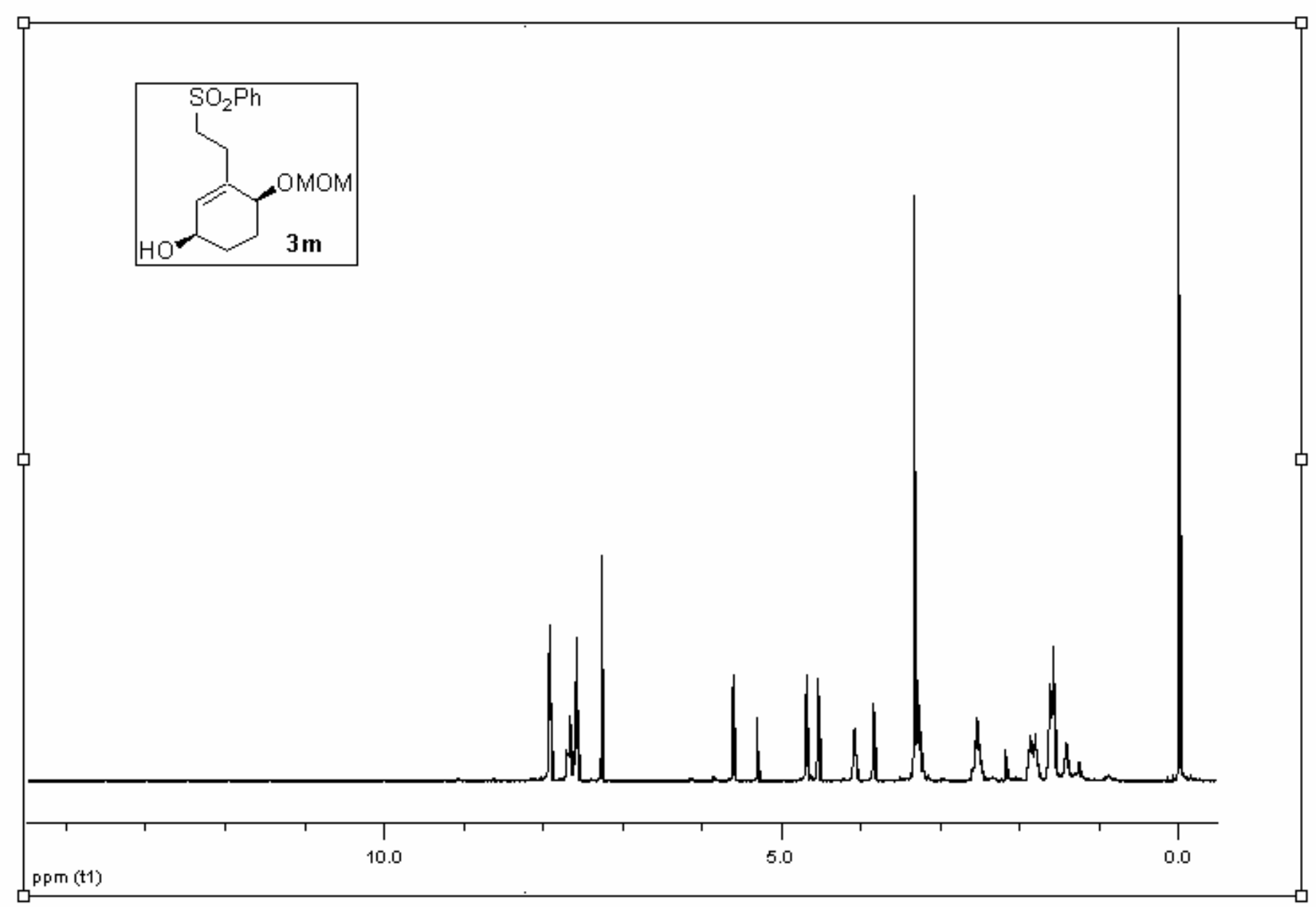




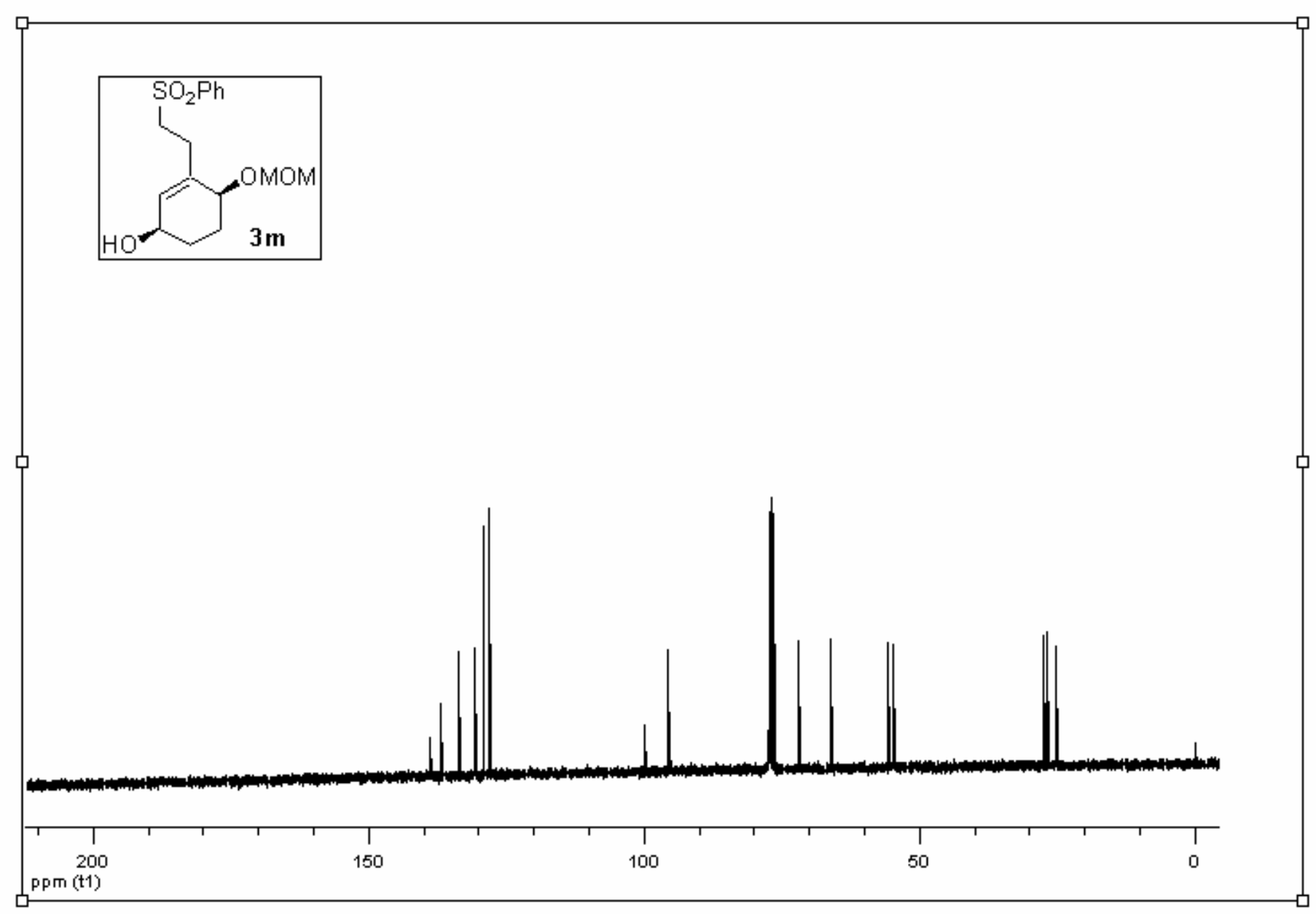

SI44 


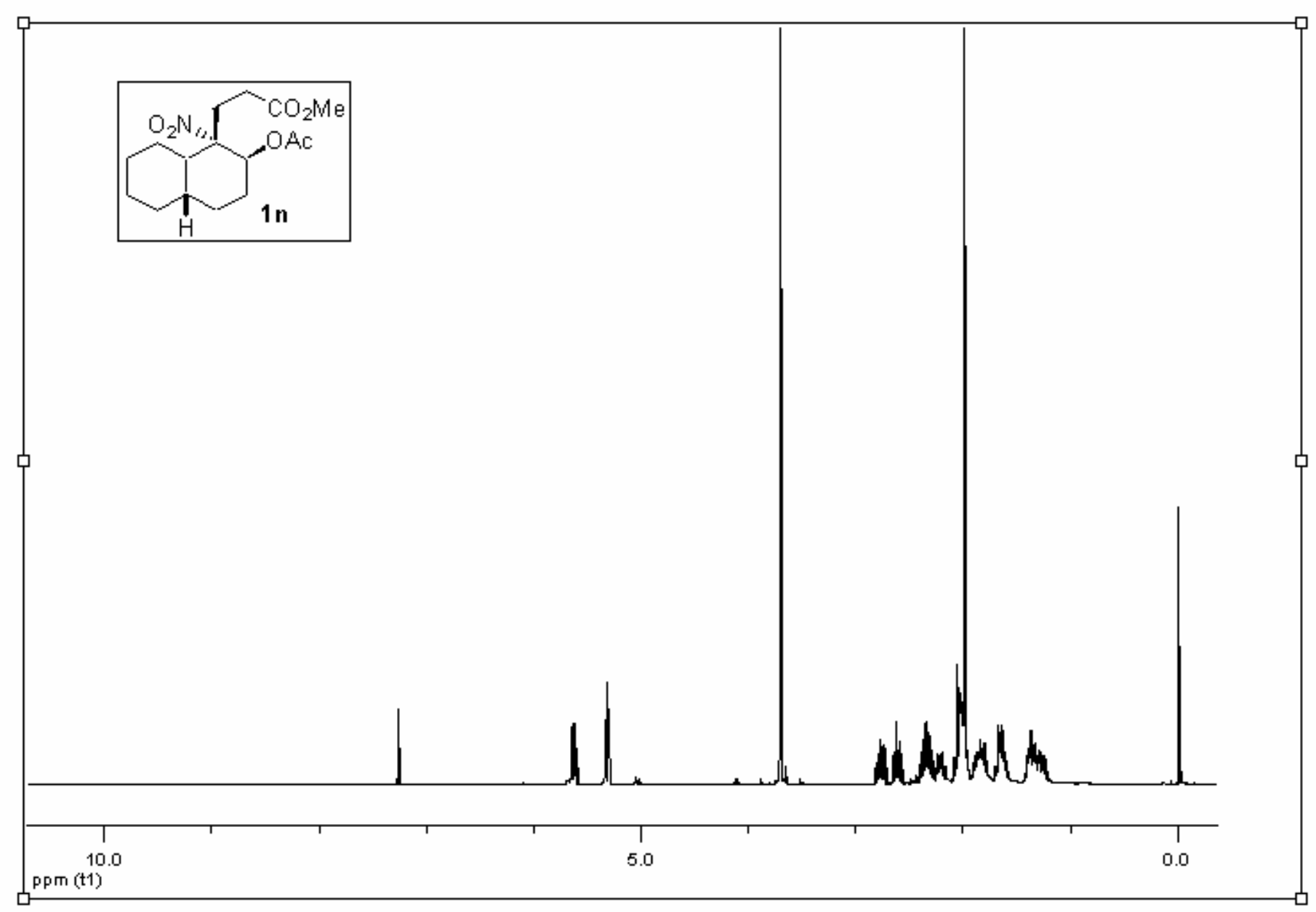




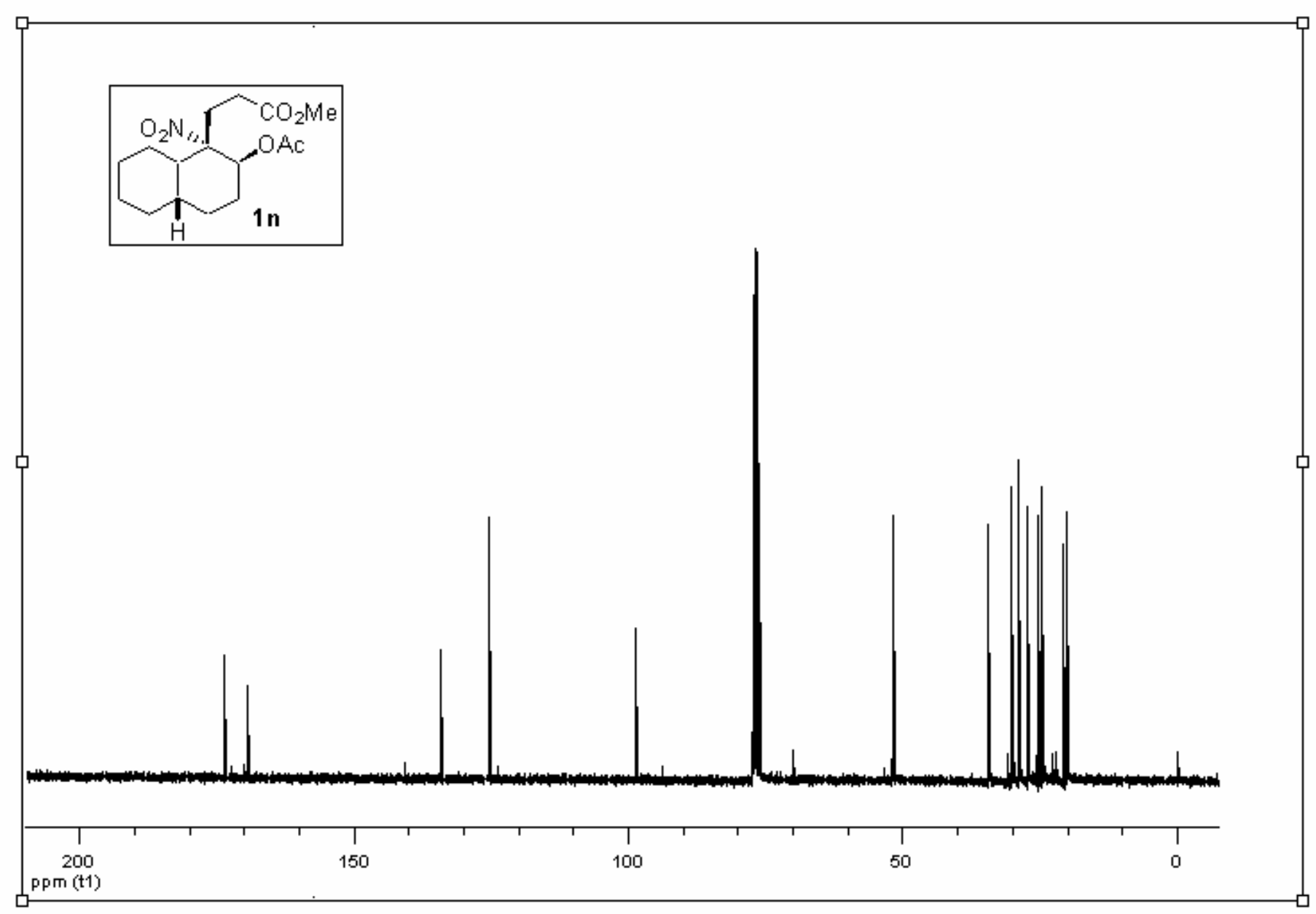




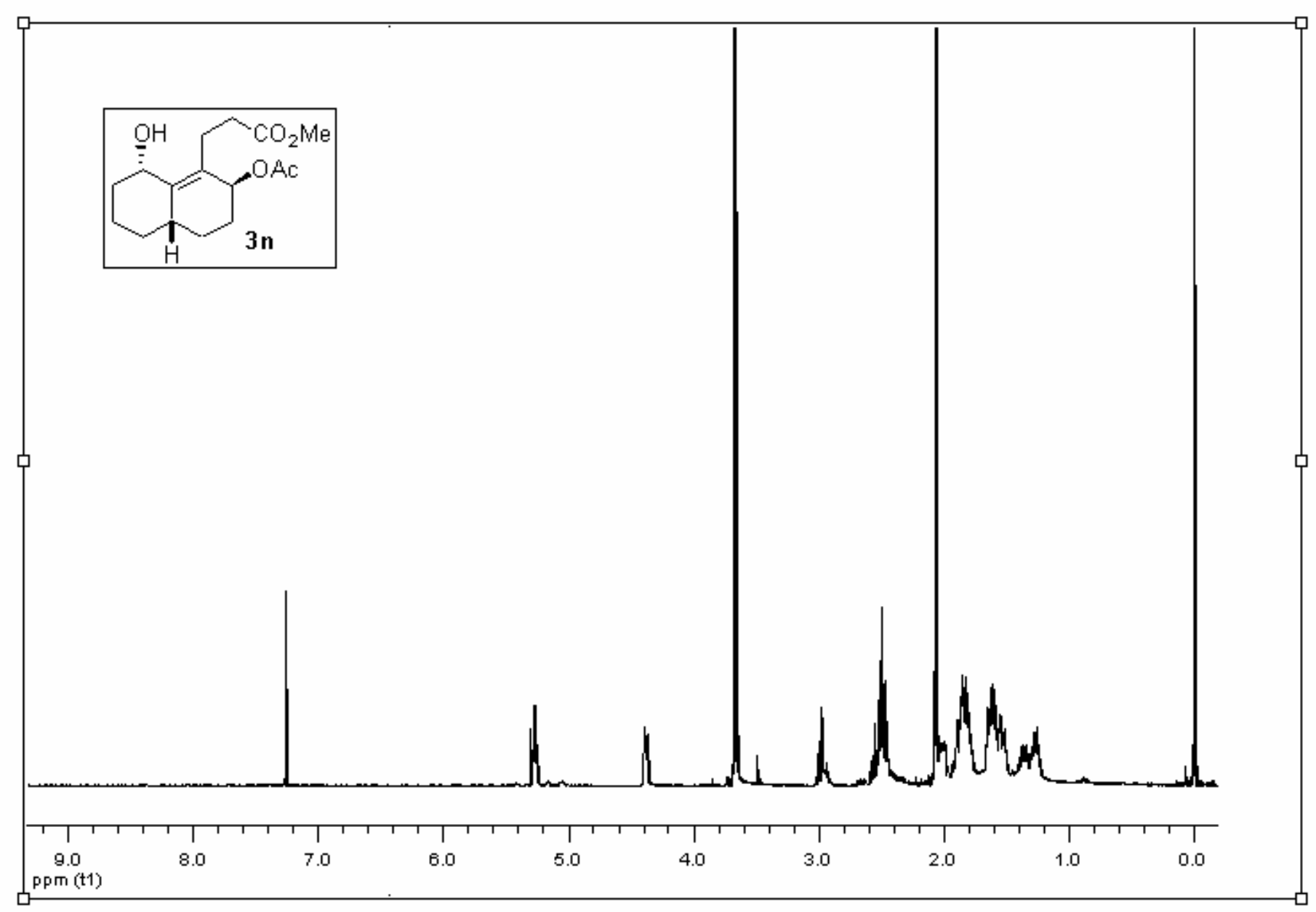




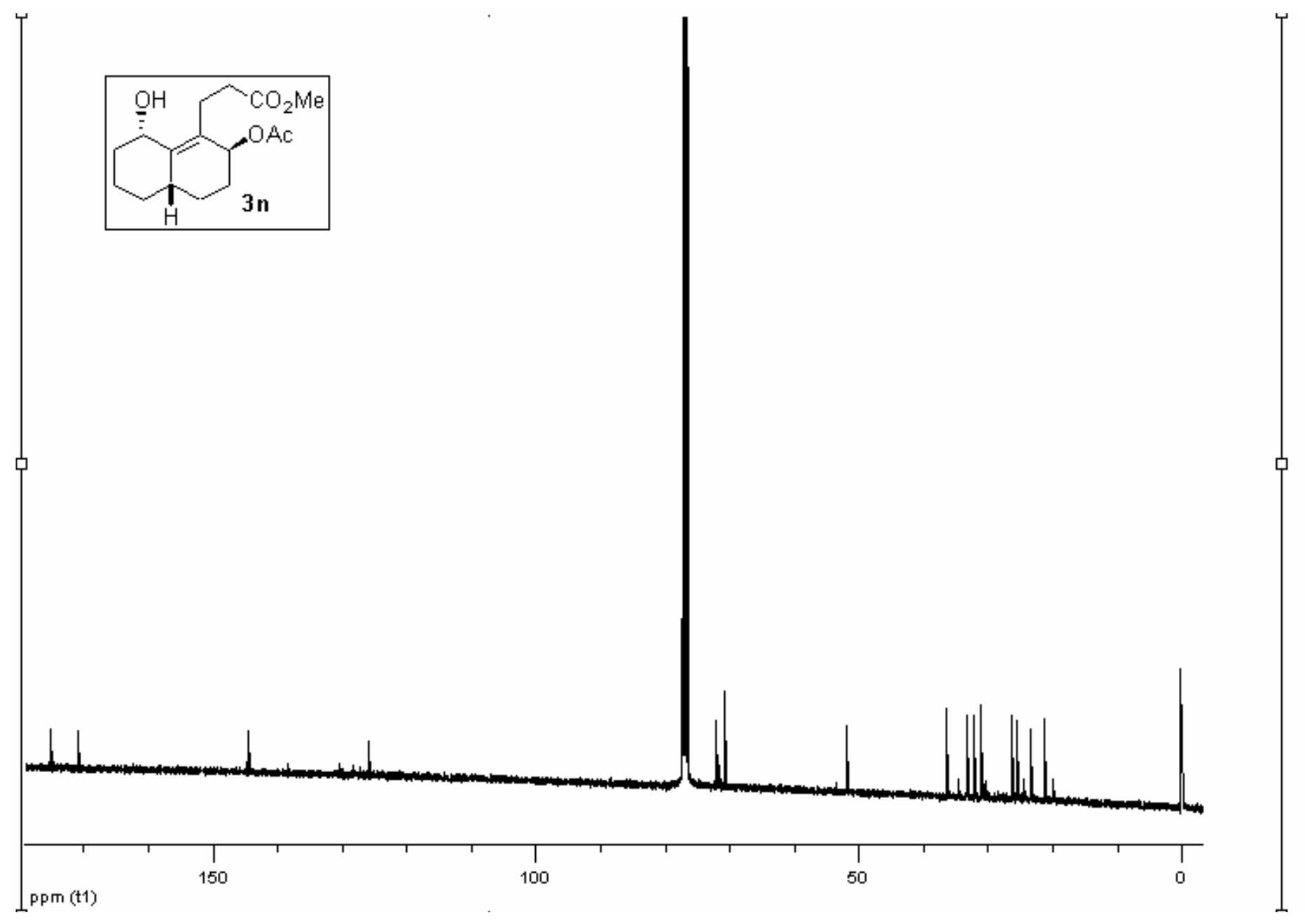

